\title{
A Discrete Invitation to Quantum Filtering and Feedback Control*
}

\section{Luc Bouten ${ }^{\dagger}$ \\ Ramon van Handel ${ }^{\dagger}$ \\ Matthew R. James ${ }^{\ddagger}$}

Dedicated to Slava Belavkin in the year of his 60th birthday

Key words. discrete quantum filtering, quantum feedback control, quantum probability, conditional expectation, dynamic programming, stochastic Lyapunov functions

AMS subject classifications. 93E11, 93E15, 93E20, 81P15, 81S25, 34F05

DOI. $10.1137 / 060671504$

I. Introduction. Control theory, and in particular feedback control, is an important aspect of modern engineering. It provides a set of tools for the design of technology with reliable performance and has been applied with overwhelming success in the design of many of the devices that we use on a daily basis. In this article we will explore the following question: rather than controlling, say, a jet engine, can we use feedback to control an object as small as a single atom?

Though it is not directly visible in our everyday lives, the technology to manipulate matter at the level of single atoms and photons is well in place and is progressing at a fast rate. Nobel prize-winning technologies such as laser cooling and trapping

* Received by the editors October 4, 2006; accepted for publication (in revised form) November 28, 2007; published electronically May 4, 2009.

http://www.siam.org/journals/sirev/51-2/67150.html

$\dagger$ Physical Measurement and Control 266-33, California Institute of Technology, Pasadena, CA 91125 (bouten@its.caltech.edu, ramon@its.caltech.edu). The work of these authors was partially supported by the Army Research Office under grants DAAD19-03-1-0073 and W911NF-06-1-0378, and by the National Science Foundation under grants CCF-0323542 and PHY-0456720.

$\ddagger$ Department of Engineering, Australian National University, Canberra, ACT 0200, Australia (matthew.james@anu.edu.au). The work of this author was supported by the Australian Research Council. 


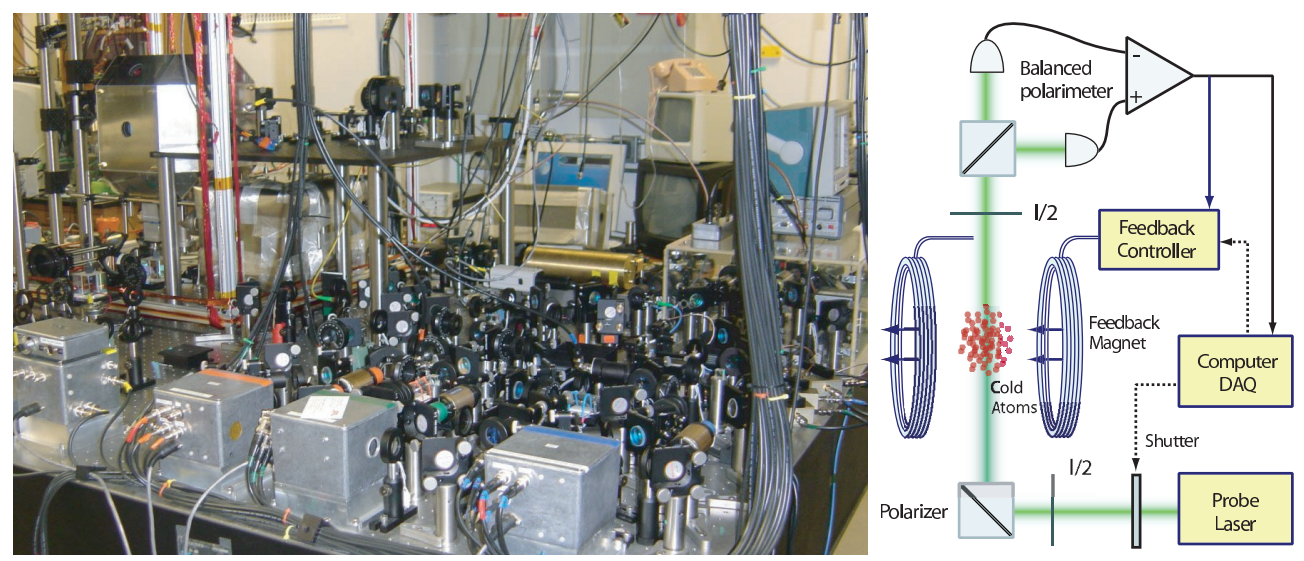

Fig. I.I A laboratory experiment in quantum feedback control, implementing the setup reported in [38]. The metal boxes in the foreground are diode lasers, whose output light is manipulated using a large number of optical elements. In the background on the left is a magnetically shielded chamber containing magnetic coils and a cell with laser cooled Cesium atoms. A schematic of the experiment is shown on the right. (Photograph courtesy of John Stockton.)

of atoms, which were state of the art only a decade ago, are now routine procedures which are being applied in physics laboratories around the world. The generation of highly coherent light and the detection of single photons has been refined since the development of the laser. Given the availability of this unprecedented level of control over the microscopic world, the question of control design seems a timely one - one could wonder whether we can "close the loop" and make the atoms do useful work for us. This is the domain of quantum feedback control.

"Quantum," of course, refers to the theory of quantum mechanics, which comes in necessarily at this level of description. The feedback which we will use to control a quantum system is a function of observations obtained from that system, and observations in quantum mechanics are inherently random. This makes the theory both challenging and interesting from the points of view of fundamental physics and control theory. As we will demonstrate, the theory of quantum feedback control resembles closely, and follows directly in many parts, the classical theory of stochastic control (here and below, classical means non-quantum mechanical).

Several promising applications of quantum feedback control have been proposed and are now starting to be investigated in a laboratory setting. One class of such applications falls under the general heading of precision metrology: can we utilize the sensitivity of small numbers of atoms to external perturbations to design ultrafast, precise sensors, when the desired accuracy is on the order of the intrinsic quantum uncertainty of the sensor? Concrete applications include, e.g., precision magnetometry $[77,78]$, which was recently investigated in a laboratory experiment [38] (see Figure 1.1), and atomic clocks [2]. A second class of examples involves the design of optical communication systems where each bit is encoded in a pulse containing only a small number of photons [28, 37]; see [3] for a related experimental demonstration. As a third application, we mention the use of feedback for cooling of atoms or nanomechanical devices [30, 46, 76]; see [23] for a laboratory demonstration (the availability of efficient cooling techniques is important in experimental physics).

As both the theory and applications are still in their infancy, it is far from clear at this time what the ultimate impact of quantum feedback control will be; it is clearly 
unlikely that future generations of washing machines or airplanes will be powered by quantum control, but other application areas, such as those mentioned above, might well benefit from the availability of such technology. At the very least, however, the questions that arise in the subject prompt us to explore the fundamental limits on the control and engineering of physical systems, and a fruitful interplay between control theory and experimental physics enables the exploration of these limits using real-life experiments such as the one displayed in Figure 1.1. In this article we demonstrate the underlying theory using a simple but rich toy model; we hope that the reader is sufficiently intrigued to come along for the ride!

The study of quantum feedback control was pioneered by V.P. Belavkin in a 1983 paper [9], long before the experimental realization of such a setup was realistically feasible. Belavkin realized that due to the unavoidable presence of uncertainty in quantum measurements, the theory of filtering - the extraction of information from noisy observations - plays a fundamental role in quantum feedback control, exactly as in the theory of stochastic control with partial observations [13]. In [9] feedback control was explored in discrete time using the operational formalism of Davies [27], which is the precursor of quantum filtering theory. Belavkin developed further the theory of quantum filtering in a series of articles $[8,10,12]$, and in [10] the use of dynamic programming for feedback control in continuous time was sketched. We refer the reader to [20] for a modern introduction to quantum filtering theory.

Independently from Belavkin's work, the equations of quantum nonlinear filtering were developed in the physics literature by Carmichael [24], based on the work of Davies [27], under the name of "quantum trajectory theory." Though the connection to filtering theory was not realized until much later, Carmichael's work nonetheless spawned an investigation on the use of feedback control in the physics literature (see, e.g., [31] and the references therein). It is here that most of the practical applications, such as the ones cited above, were proposed and developed.

Like its classical counterpart, the mathematical theory of quantum filtering and stochastic control in continuous time can be quite technical, requiring a heavy analytic machinery to obtain the necessary results in a rigorous way. On the other hand, we believe that the methods in the field can be understood perfectly well without being obscured by analytic complications. What we are going to do in this paper is to give a complete development of the basic theory of quantum filtering and feedback control in a simple toy model, which requires essentially no analytic machinery. We assume only some background in linear algebra and familiarity with elementary probability theory with martingales, roughly at the level of the inspiring textbook by D. Williams [83].

The model we will investigate probably has a familiar ring to those readers who are familiar with mathematical finance. It is in fact little more than a noncommutative version of the binomial model which has enjoyed wide popularity since its introduction by Cox, Ross, and Rubinstein [26]. The model is widely used in the teaching of mathematical finance [75] and even on Wall Street as a flexible computational tool for the pricing of financial derivatives. The model has three key features, all of which are shared by its noncommutative counterpart, which make its investigation particularly appealing: (i) on the one hand, the model is as simple as it can possibly be, consisting of a finite number of two-state random variables (coin flips) - hence analytical complications are avoided; (ii) on the other hand, many of the mathematical tools that are indispensable in the continuous context can be developed within the framework of this model and admit simple proofs; in particular, change of measure techniques, the martingale representation theorem, discrete stochastic integrals, and even a (trivial) stochastic calculus can all be demonstrated in this context; and (iii) 
the model is in fact an approximation of the usual continuous time models in the sense that these are recovered when the limit as the time step tends to zero is taken in a suitable sense. For these reasons, we believe that such models are ideally suited as a first introduction to quantum filtering and feedback control.

The noncommutative model has an independent history in the quantum probability literature. In his book [68], P.-A. Meyer uses the building blocks of the model we use in this article to demonstrate the basic features of the Fock space, which underlies the continuous quantum stochastic calculus of Hudson-Parthasarathy [49], in a discrete setting (he calls this model, which he attributes to Journé [68, page 18], the "toy Fock space"). Repeated interaction models of a similar type were considered by Kümmerer [61] and by Lindsay and Parthasarathy [63], and the similarities between discrete and continuous stochastic calculus were further explored by Attal [5] and by Pautrat [72]. Various authors have considered the continuous limit of such models $[63,5,6,41]$ and have demonstrated convergence of the associated discretized evolution equations to (Hudson-Parthasarathy) quantum stochastic differential equations that are routinely used for the description of realistic physical systems [36]. The description of measurements (in essence, filtering) in this discrete framework was deduced from basic physical arguments in Brun [22] and Gough and Sobolev [43].

In this paper we will not take the simplest and most straightforward route in treating these discrete models; similar equations can often be deduced by more elementary means $[22,43]$. Rather, we will exploit the richness of the model to mimic as closely as possible the development of quantum filtering and feedback control in the continuous setting, where simple arguments based on linear algebra are useless. Indeed, the introduction of a "trivial" stochastic calculus, martingale representation, etc., may seem to be overkill in the context of this model, but these become powerful and indispensable tools in the treatment of the more general (and physically relevant) framework of continuous time evolution and observations. We hope to provide in this way a (relatively) gentle introduction to the full technical theory, where all the necessary ingredients make their appearance in a more accessible setting.

Organization of the Paper. Despite our best efforts, this paper has become a lengthy one. In order to help the reader navigate through the material, we now provide a brief road map of coming attractions.

The paper consists of three parts. The first part aims to provide an introduction to quantum probability theory and to build simple discrete counterparts of quantum stochastic models and quantum stochastic calculus. The second part is devoted to developing nonlinear filtering theory in the quantum setting. The third part of the paper is devoted to the application of these ideas to quantum stochastic control.

The first part of the paper consists of sections $2-4$. In section 2 we introduce the basic notions of quantum probability and develop in detail the quantum binomial model which will be employed throughout the paper. The latter is a physical model, and the following two sections develop some mathematical techniques which we will use to manipulate it. Section 3 introduces conditional expectations in the quantum context and sets up the filtering problem which will be studied in the second part. In section 4 we develop a discrete stochastic calculus and use it to express the model of section 2 in the form of a difference equation.

The second part of the paper consists of sections 5 and 6 . Section 5 treats filtering using martingale methods, while section 6 uses change of measure techniques. Both of these techniques are of significant interest in the filtering problem, but they give rise to the same equations. The reader is encouraged to skip section 6 on first reading, as it is not needed in the remainder of the paper. 
The third part of the paper consists of sections $7-9$. In section 7 we show how to incorporate feedback into the model of section 2. The question that remains to be answered is how to design a suitable feedback strategy, given a particular control goal. There are various ways of doing this, and two possibilities are given in the following sections. In section 8 we develop optimal feedback controls using dynamic programming techniques, while section 9 explores an alternative control design method using Lyapunov functions. Sections 8 and 9 can be read independently.

Finally, we conclude in section 10 with extensive suggestions for further reading.

Notation. We use the following conventions. In general, Hilbert spaces are denoted by the symbol $\mathrm{H}$ and are endowed with the inner product $\langle\cdot, \cdot\rangle$. We will assume all Hilbert spaces are complex and finite-dimensional. Recall that the adjoint of a linear operator $X: \mathrm{H} \rightarrow \mathrm{H}$ is defined as the unique operator $X^{*}$ that satisfies $\left\langle X^{*} x, y\right\rangle=\langle x, X y\rangle \forall x, y \in \mathrm{H}$, and that $X$ is called self-adjoint if $X=X^{*}$. We denote by $I$ the identity operator. The commutator between two linear operators is denoted as $[X, Y]=X Y-Y X$. Calligraphic letters will be used for various purposes, such as classical $\sigma$-algebras (e.g., $\mathcal{Y}$ ) or functionals on an algebra (e.g., $\mathcal{L}(X)$ ). Control sets and related quantities are denoted by gothic type (e.g., $\mathfrak{U}$ ). ${ }^{*}$-algebras (to be defined below) will be denoted by script type $\mathscr{A}$, and states on such an algebra are often denoted by blackboard bold type (e.g., $\mathbb{P}$ ), though $\rho$ and $\phi$ will also be used. The generic classical probability space will be denoted as $(\Omega, \mathcal{F}, \mathbf{P}), \mathbf{E}_{\mathbf{P}}$ denotes the expectation with respect to the measure $\mathbf{P}$, and $\ell^{\infty}(\mathcal{F})$ denotes the space of $\mathcal{F}$-measurable (bounded, but trivially so when $\Omega$ is a finite set) random variables.

As is unavoidable in an article of this length, there is much notation that is introduced throughout the article and that is constantly reused. To help the reader to keep track of the various quantities, we have provided below a list of commonly used objects, a brief description, and the page number on which the object is defined.

$A(l), A^{*}(l), \Lambda(l), t(l)$ Discrete noises $\quad 251$

$J(\mu) \quad$ Control cost function 296

$\begin{array}{lll}L_{1,2,3} & \text { Interaction operators } & 254\end{array}$

$M(l) \quad$ Single time step interaction 255

$M^{\mu}(l, u) \quad$ Controlled single time step interaction 290

$M^{\circ,+,-, \pm} \quad$ Coefficients in difference equation for $U(l) \quad 265$

$M_{l} \quad$ Single time step interaction, time reversed 255

$M_{l}(u) \quad$ Controlled single time step interaction, time reversed 289

$U(l) \quad$ Interaction unitary 255

$U(l, \mathbf{u}) \quad$ Interaction unitary with open loop control $\quad 289$

$U^{\mu}(l) \quad$ Interaction unitary with feedback control 290

$V(l) \quad$ Change of state operator for reference measure 282

$V_{l}(\rho) \quad$ Value function of dynamic programming 299

$\begin{array}{lll}W_{l}(\mu, \rho) & \text { Cost-to-go } & 298\end{array}$

$Y(l) \quad$ Observation process 256

$Y^{\mu}(l) \quad$ Observations process for control strategy $\mu \quad 292$

$Z(l) \quad$ Field process with $Y(l)=U(l)^{*} Z(l) U(l) \quad 256$

$\Delta L(l) \quad L(l)-L(l-1) \quad 263$

$\Phi \quad$ Vacuum vector in $\mathbb{C}^{2} \quad 248$

$\iota \quad{ }^{*}$-isomorphism between observables and random variables 247

$\begin{array}{lll}\lambda^{2} & \text { Length of each time slice } & 250\end{array}$

$\mathbb{P} \quad$ Standard state on the atom-field algebra $\mathscr{M} \otimes \mathscr{W}_{k} \quad 254$ 


$\begin{array}{lll}\mathcal{J}(X) & \mathcal{J} \text {-coefficient of the filtering equations } & 273 \\ \mathcal{L}(X) & \text { Discrete Lindblad generator } & 265 \\ \mathcal{T}(X) & \mathcal{T} \text {-coefficient of the filtering equations } & 286 \\ \mathcal{Y}_{l} & \text { Classical observation filtration } & 256 \\ \mathfrak{K}_{\mathfrak{K}} & \text { Set of admissible controls } & 290 \\ \mathfrak{K}_{S} & \text { Set of admissible separated controls } & 295 \\ \mathfrak{U} & \text { Control set } & 289 \\ \check{\mathfrak{u}}_{l} & \text { Control process } f_{l}(\Delta Z(1), \ldots, \Delta Z(l-1)) & 291 \\ \mathfrak{u}_{l} & \text { Control signal } f_{l}(\Delta Y(1), \ldots, \Delta Y(l-1)) & 290 \\ \mathscr{B}_{l} & \text { Filtration in } \mathscr{M} \otimes \mathscr{W}_{k} & 263 \\ \mathscr{C}_{l} & \text { Filtration of } Z & 256 \\ \mathscr{M} & \text { Two-level system algebra } & 248 \\ \mathscr{M}_{l} & \text { Field algebra of time slice } l & 250 \\ \mathscr{W}_{k} & \text { Field algebra } & 250 \\ \mathscr{Y}_{l} & \text { Observation filtration } & 256 \\ \mu, \mu^{*}, \bar{\mu} & \text { Control strategies } & 290 \\ \phi & \text { Vacuum state on } \mathscr{M} & 251 \\ \pi_{l}(X) & \text { Conditional state (filtered estimate of } X) & 262 \\ \sigma_{ \pm}, \sigma_{z} & \text { Pauli matrices in } \mathscr{M} & 248 \\ \sigma_{l}(X) & \text { Unnormalized conditional state } & 283 \\ \tilde{Y}(l) & \text { Innovations process } & 270 \\ f_{l}(\cdots) & \text { Feedback function } & 290 \\ g_{l}(\rho) & \text { Separated feedback function } & 295 \\ j_{l}(X) & \text { Time evolution of } X & 255 \\ j_{l}^{\mathbf{u}}(X) & \text { Time evolution with open loop control } & 289 \\ j_{l}^{\mu}(X) & \text { Time evolution with feedback control } & 290 \\ k & \text { Number of time slices } & 250 \\ u_{l}^{\mu} & \text { Classical feedback signal for control strategy } \mu & 294 \\ y_{l}^{\mu} & \text { Classical observations process for control strategy } \mu & 294 \\ y_{l} & \text { Classical observation process } & 256 \\ & & \end{array}$

2. The Quantum Binomial Model. In this section we introduce the basic model that we will be dealing with throughout the paper: a discretized approximation of the interaction between an atom and the electromagnetic field. First, however, we need to demonstrate how probability theory fits into the framework of quantum mechanics.

2.I. Random Variables in Quantum Mechanics. The basic setting of quantum mechanics, as one would find it in most textbooks, is something like this. We start with a Hilbert space $\mathrm{H}$ and fix some "state vector" $\psi \in \mathrm{H}$. An "observable," the physicist's word for random variable, is described by a self-adjoint operator $X$ on $\mathrm{H}$, and the expectation of $X$ is given by $\langle\psi, X \psi\rangle$. The set of values that $X$ can take in a single measurement is its set of eigenvalues, and the probability of observing the eigenvalue $\lambda_{i}$ is given by $\left\langle\psi, P_{i} \psi\right\rangle$, where $P_{i}$ is the projection operator onto the eigenspace corresponding to $\lambda_{i}$. This is quite unlike the sort of description we are used to from classical probability theory - or is it?

In fact, the two theories are not as dissimilar as they may seem, and it is fruitful to formalize this idea (we will do so in the next section). The key result that we need is the following elementary fact from linear algebra. This is just the statement that a set of commuting normal $\left(\left[X, X^{*}\right]=0\right)$ matrices can be simultaneously diagonalized; see, e.g., [47, sect. 2.5] or [44, sect. 84]. 
TheOREm 2.1 (spectral theorem). Let $\mathrm{H}$ be an $n$-dimensional Hilbert space, $n<\infty$. Let $\mathscr{C}$ be a set of linear transformations from $\mathrm{H} \rightarrow \mathrm{H}$ that is closed under the adjoint (i.e., if $C \in \mathscr{C}$, then also $C^{*} \in \mathscr{C}$ ) and such that all the elements of $\mathscr{C}$ commute (i.e., $[C, D]=0 \forall C, D \in \mathscr{C}$ ). Then there exists an orthonormal basis of $\mathrm{H}$ such that every $C \in \mathscr{C}$ is represented by a diagonal matrix with respect to this basis.

Let us demonstrate how this works in the simplest case. Let $\operatorname{dim} \mathrm{H}=2$, fix some $\psi \in \mathrm{H}$, and let $X=X^{*}$ be a self-adjoint operator on $\mathrm{H}$. The set $\mathscr{C}=\{X\}$ satisfies the conditions of the spectral theorem, so we can find an orthonormal basis in $\mathrm{H}$ such that we can express $X$ and $\psi$ in this basis as

$$
X=\left(\begin{array}{cc}
x_{1} & 0 \\
0 & x_{2}
\end{array}\right), \quad \psi=\left(\begin{array}{l}
\psi_{1} \\
\psi_{2}
\end{array}\right) .
$$

We can now interpret $X$ as a random variable on some probability space. Introduce $\Omega=\{1,2\}$, the map $x: \Omega \rightarrow \mathbb{R}, x(i)=x_{i}$, and the probability measure $\mathbf{P}(\{i\})=\left|\psi_{i}\right|^{2}$ $(i=1,2)$. Evidently $\langle\psi, X \psi\rangle=\mathbf{E}_{\mathbf{P}}(x)$, i.e., the random variable $x$ has the same expectation under $\mathbf{P}$ as we obtain from $X$ and $\psi$ using the usual quantum mechanical formalism. We can also easily calculate $\mathbf{P}\left(x=x_{i}\right)=\left|\psi_{i}\right|^{2}$, which is again consistent with the rules of quantum mechanics. As the spectral theorem works for more general sets $\mathscr{C}$, we can follow the same procedure to represent a set of commuting observables on a classical probability space and to calculate the joint statistics.

Up to this point we have done nothing particularly exciting: all we have done is to express the quantum "rules" listed in the first paragraph of this section in a convenient basis, and we have attached an interpretation in terms of classical probability theory. Conceptually, however, this is an important point: commuting observables are random variables on a classical probability space, and should be thought of in that way. Formalizing the way we can pass back and forth between the two descriptions will allow us to utilize classical probabilistic techniques in the quantum mechanical setting.

What the spectral theorem does not allow us to do is to simultaneously interpret two noncommuting observables as random variables on a single probability space. This is not a shortcoming of the theory, but has an important physical meaning. Observables that do not commute cannot be observed in the same realization: their joint probability distribution is a meaningless quantity (hence they are called incompatible). Strange as this may seem, this idea is a cornerstone of quantum theory and all the empirical evidence supports the conclusion that this is how nature works. We will accept as a physical principle that in a single realization we can choose to measure at most a commuting set of observables. However, we will see later that we can still estimate the statistics of observables which we did not choose to measure, even if they do not commute with each other, provided they commute with the measurements we did choose to perform. These ideas will be clarified in due course.

2.2. Quantum Probability Spaces. In this section we introduce and motivate the notion of a (finite-dimensional) quantum probability space. The definitions may not seem completely natural at first sight, but their use will be amply illustrated in the remainder of the article.

In the example of the previous section, we saw how to construct a set of quantum observables as maps on the sample space $\Omega$. To turn this into a probability space we need to add a $\sigma$-algebra and a probability measure. As we are considering a particular set of random variables $X_{1}, \ldots, X_{k}$ it is natural to choose the $\sigma$-algebra $\sigma\left(X_{1}, \ldots, X_{k}\right)$, i.e., the smallest $\sigma$-algebra with respect to which $X_{1}, \ldots, X_{k}$ are measurable (the $\sigma$-algebra generated by $X_{1}, \ldots, X_{k}$ ), and the quantum state induces a 
probability measure on this $\sigma$-algebra. We would like to be able to specify the $\sigma$ algebra directly, however, without having to select some "preferred" set of random variables $X_{1}, \ldots, X_{k}$-the values taken by these random variables are irrelevant, as we are only interested in the generated $\sigma$-algebra.

The definition we will give is motivated by the following observation. On a classical sample space $\Omega$, consider the set $\ell^{\infty}(\mathcal{F})$ of all random variables measurable with respect to the $\sigma$-algebra $\mathcal{F}$. If one is given such a set, then $\mathcal{F}$ can be reconstructed as $\mathcal{F}=\sigma\left\{\ell^{\infty}(\mathcal{F})\right\}$. Conversely, however, we can easily construct $\ell^{\infty}(\mathcal{F})$ if we are given $\mathcal{F}$. Hence these two structures carry the same information; by considering all measurable random variables at the same time we are no longer giving preference to a particular set $X_{1}, \ldots, X_{k}$. In quantum probability, it will be more convenient to characterize a $\sigma$-algebra by its $\ell^{\infty}$-space - the quantum analogue of this object is a set of observables $\mathscr{A}$, which can be used directly as input for the spectral theorem. To complete the picture we need a way to characterize sets of observables $\mathscr{A}$ which are mapped by the spectral theorem to a space of the form $\ell^{\infty}(\mathcal{F})$.

Definition 2.2. $A^{*}$-algebra $\mathscr{A}$ is a set of linear transformations $\mathrm{H} \rightarrow \mathrm{H}$ such that $I, \alpha A+\beta B, A B, A^{*} \in \mathscr{A}$ for any $A, B \in \mathscr{A}, \alpha, \beta \in \mathbb{C}$. $\mathscr{A}$ is called commutative if $A B=B A$ for any $A, B \in \mathscr{A}$. A linear map $\rho: \mathscr{A} \rightarrow \mathbb{C}$ which is positive $\rho\left(A^{*} A\right) \geq 0$ $\forall A \in \mathscr{A}$ and normalized $\rho(I)=1$ is called a state on $\mathscr{A}$.

Definition 2.3. $A^{*}$-isomorphism between a commutative*-algebra $\mathscr{A}$ and a set of functions $\mathcal{A}$ on some space $\Omega$ is a linear bijection $\iota: \mathscr{A} \rightarrow \mathcal{A}$ such that $\iota\left(A^{*}\right)=$ $\iota(A)^{*}\left(\iota(A)^{*}(i)\right.$ is the complex conjugate of $\left.\iota(A)(i) \forall i \in \Omega\right)$ and $\iota(A B)=\iota(A) \iota(B)$ $((\iota(A) \iota(B))(i)=\iota(A)(i) \iota(B)(i) \forall i \in \Omega)$ for every $A, B \in \mathscr{A}$.

The reason we want ${ }^{*}$-isomorphisms is to ensure that we can manipulate observables and classical random variables in the same way. That is, if $X_{1}, X_{2}$ are commuting self-adjoint operators that correspond to the random variables $x_{1}, x_{2}$, then $X_{1}+X_{2}$ must correspond to $x_{1}+x_{2}$ and $X_{1} X_{2}$ must correspond to $x_{1} x_{2}$. The notion of a *-algebra implements exactly the question posed above: every commutative ${ }^{*}$-algebra can be mapped to a set of random variables of the form $\ell^{\infty}(\mathcal{F})$ for some $\sigma$-algebra $\mathcal{F}$ by using the spectral theorem and the following lemma. The fact that sets of measurable functions can be characterized by algebras is well known; in fact, Lemma 2.4 is just a "trivial" version of the monotone class theorem [73, Chap. I, Thm. 8].

Lemma 2.4. Let $\Omega=\{1, \ldots, n\}$. Then there is a one-to-one correspondence between (commutative) ${ }^{*}$-algebras of $n \times n$ diagonal matrices and $\ell^{\infty}(\mathcal{F})$-spaces.

Proof. We need two simple facts. First, let $X_{1}, \ldots, X_{k}$ be elements of a *-algebra of diagonal matrices. Then $f\left(X_{1}, \ldots, X_{k}\right)$ is also an element of the ${ }^{*}$-algebra for any function $f$. Clearly this is true if $f$ is any (complex) polynomial. But this is sufficient, because given a finite number of points $x_{1}, \ldots, x_{n} \in \mathbb{C}^{k}$ we can always find for any function $f$ a polynomial $\hat{f}$ that coincides with $f$ on $x_{1}, \ldots, x_{n}$. Second, we claim that for any $\mathcal{F}$ there exists a finite set of disjoint sets $S_{1}, \ldots, S_{k} \in \mathcal{F}$ such that $\bigcup_{k} S_{k}=\Omega$ and such that any $x \in \ell^{\infty}(\mathcal{F})$ can be written as $x=\sum_{i} x_{i} \chi_{S_{i}}\left(\chi_{S}\right.$ is the indicator function on $S, x_{i} \in \mathbb{C}$ ). To see this, note that there is only a finite possible number of disjoint partitions $\left\{T_{i}\right\}$ of $\Omega$, the latter being a finite set. $\left\{S_{i}\right\}$ is then the (unique) finest such partition that is a subset of $\mathcal{F}$. To show uniqueness, if $\left\{S_{i}\right\},\left\{S_{i}^{\prime}\right\} \subset \mathcal{F}$ were two such partitions, then $\left\{S_{i} \cap S_{j}^{\prime}\right\} \subset \mathcal{F}$ would be a finer partition unless $\left\{S_{i}\right\}=\left\{S_{i}^{\prime}\right\}$.

Let us now prove the lemma. For a diagonal matrix $X$ we define the map $x(i)=$ $\iota(X)(i)=X_{i i}$, and similarly for a map $x: \Omega \rightarrow \mathbb{C}$ define the diagonal matrix $X_{i i}=$ $\iota^{-1}(x)_{i i}=x(i)$. This gives a ${ }^{*}$-isomorphism $\iota$ between the set $\mathscr{A}$ of all diagonal matrices and the set $\ell^{\infty}(\Omega)$ of all maps on $\Omega$. The claim of the lemma is that $\iota$ maps any ${ }^{*}$-subalgebra $\mathscr{C} \subset \mathscr{A}$ to $\ell^{\infty}(\mathcal{F})$ for some $\sigma$-algebra $\mathcal{F}$, and conversely that 
$\iota^{-1}\left(\ell^{\infty}(\mathcal{F})\right)$ is a ${ }^{*}$-algebra for any $\mathcal{F}$. The latter is a straightforward consequence of the definitions, so let us prove the former statement. Fix the ${ }^{*}$-algebra $\mathscr{C}$ and define $\mathcal{C}=\iota(\mathscr{C})$. Let $\mathcal{F}=\sigma\{\mathcal{C}\}$, and introduce the smallest $\Omega$-partition $\left\{S_{i}\right\} \subset \mathcal{F}$ as above. If $\chi_{S_{i}} \in \mathcal{C}$ for every $i$, then we must have $\mathcal{C}=\ell^{\infty}(\mathcal{F}): \mathcal{C}$ is an algebra and hence contains all random variables of the form $\sum_{i} x_{i} \chi_{S_{i}}$. Let us thus assume that there is some $i$ such that $\chi_{S_{i}} \notin \mathcal{C}$; as $\mathcal{F}=\sigma\{\mathcal{C}\}$, however, we must be able to find functions $x_{(1)}, \ldots, x_{(n)} \in \mathcal{C}$ such that $x_{(1)}^{-1}\left(x_{1}\right) \cap \cdots \cap x_{(n)}^{-1}\left(x_{n}\right)=S_{i}$. But if we choose $f\left(y_{1}, \ldots, y_{n}\right)=\chi_{\left\{y_{1}=x_{1}, \ldots, y_{n}=x_{n}\right\}}\left(y_{1}, \ldots, y_{n}\right)$, then $f\left(x_{(1)}, \ldots, x_{(n)}\right)=\chi_{S_{i}} \in \mathcal{C}$. Hence we have a contradiction, and the lemma is proved.

We are now ready to introduce the basic mathematical object studied in this article: a generalized or quantum probability space. Applying the spectral theorem to this structure gives a fundamental result which we will use over and over.

Definition 2.5 (quantum probability space). The pair $(\mathscr{A}, \rho)$, where $\mathscr{A}$ is a *-algebra of operators on a finite-dimensional Hilbert space $\mathrm{H}$ and $\rho$ is a state on $\mathscr{A}$, is called a (finite-dimensional) quantum probability space.

TheOREM 2.6 (spectral theorem for quantum probability spaces). Let $(\mathscr{C}, \rho)$ be a commutative quantum probability space. Then there is a probability space $(\Omega, \mathcal{F}, \mathbf{P})$ and $a^{*}$-isomorphism $\iota: \mathscr{C} \rightarrow \ell^{\infty}(\mathcal{F})$ such that $\rho(X)=\mathbf{E}_{\mathbf{P}}(\iota(X)) \forall X \in \mathscr{C}$.

What is so general about a generalized or quantum probability space? It is the existence of many commutative subalgebras within $(\mathscr{A}, \rho)$. Theorem 2.6 does not apply directly to $(\mathscr{A}, \rho)$, as usually such a space will not be commutative. On the other hand, in a single realization we can choose to observe at most a commutative set of observables, which generate a commutative subalgebra $\mathscr{C} \subset \mathscr{A}$. The probability space $\left(\mathscr{C},\left.\rho\right|_{\mathscr{C}}\right)$ is commutative and is thus exactly equivalent to a classical probability space by Theorem 2.6. The noncommutative probability space $(\mathscr{A}, \rho)$ describes the statistics of all possible experiments - it is a collection of many incompatible classical probability models, each of which coincides with a commutative subalgebra of $\mathscr{A}$. The experiment we choose to perform in one single realization determines which commutative subalgebra of $\mathscr{A}$, i.e., which classical probability space, is needed to describe the random outcomes of that experiment.

Remark 2.7. In section 2.1 we determined the probability measure $\mathbf{P}$ using a state vector $\psi \in \mathrm{H}$; here we have replaced this notion by the state $\rho: \mathscr{C} \rightarrow \mathbb{C}$. This is in fact a generalization: the vector $\psi$ corresponds to the state $\rho(X)=\langle\psi, X \psi\rangle$. In general we can always characterize a state $\rho$ by a density matrix $\tilde{\rho}$ as follows: $\rho(X)=\operatorname{Tr}[\tilde{\rho} X]$. This follows directly from linearity of $\rho$. Positivity and normalization impose the additional conditions $\rho \geq 0$ and $\operatorname{Tr} \rho=1$. A state of the form $\rho(X)=$ $\langle\psi, X \psi\rangle=\operatorname{Tr}\left[\left(\psi \psi^{*}\right) X\right]$ is known as a pure or vector state, whereas any other state is known as a mixed state. Both state vectors and density matrices are commonly used in the physics literature, while $\rho: \mathscr{C} \rightarrow \mathbb{C}$ is usual in quantum probability.

We will often speak of the *-algebra generated by a set of observables $X_{1}, \ldots, X_{k}$. By this we mean the smallest *-algebra $\operatorname{alg}\left\{X_{1}, \ldots, X_{k}\right\}$ of operators on $\mathrm{H}$ that contains $X_{1}, \ldots, X_{k}$. This notion plays exactly the same role as the $\sigma$-algebra generated by a set of random variables - indeed, it is straightforward to verify that if $X_{1}, \ldots, X_{k}$ commute, then $\iota\left(\operatorname{alg}\left\{X_{1}, \ldots, X_{k}\right\}\right)=\ell^{\infty}\left(\sigma\left\{\iota\left(X_{1}\right), \ldots, \iota\left(X_{k}\right)\right\}\right)$.

In the following we will need to construct quantum probability spaces from a number of independent smaller probability spaces. The analogous classical notion can be illustrated as follows: Suppose $\left(\Omega_{i}, \mathcal{F}_{i}, \mathbf{P}_{i}\right), i=1,2$, are two independent copies of the probability space of a coin flip. Then $\left(\Omega_{1} \times \Omega_{2}, \mathcal{F}_{1} \times \mathcal{F}_{2}, \mathbf{P}_{1} \times \mathbf{P}_{2}\right)$ is the joint probability space for the two independent coin flips, on which any random variable $f\left(\omega_{1}, \omega_{2}\right)=f\left(\omega_{1}\right)$, which depends only on the first coin, and $g\left(\omega_{1}, \omega_{2}\right)=g\left(\omega_{2}\right)$, 
which depends only on the second coin, are independent (under the product measure $\left.\mathbf{P}_{1} \times \mathbf{P}_{2}\right)$.

The analogous construction for quantum probability spaces uses the tensor product; that is, given quantum probability spaces $\left(\mathscr{A}_{i}, \rho_{i}\right)$ defined on the Hilbert spaces $\mathrm{H}_{i}$, we construct the joint space $\left(\mathscr{A}_{1} \otimes \mathscr{A}_{2}, \rho_{1} \otimes \rho_{2}\right)$ on the Hilbert space $\mathrm{H}_{1} \otimes \mathrm{H}_{2}$. Here $\mathrm{H}_{1} \otimes \mathrm{H}_{2}$ and $\mathscr{A}_{1} \otimes \mathscr{A}_{2}$ denote the usual vector and matrix tensor products, while $\rho_{1} \otimes \rho_{2}$ is defined by $\left(\rho_{1} \otimes \rho_{2}\right)(A \otimes B)=\rho_{1}(A) \rho_{2}(B)$ and is extended to all of $\mathscr{A}_{1} \otimes \mathscr{A}_{2}$ by linearity. It is not difficult to verify also that construction is consistent with the classical case, as $\ell^{\infty}\left(\mathcal{F}_{1} \times \mathcal{F}_{2}\right)=\ell^{\infty}\left(\mathcal{F}_{1}\right) \otimes \ell^{\infty}\left(\mathcal{F}_{2}\right)$; the ${ }^{*}$-isomorphism $\iota$ obtained through the spectral theorem then maps observables of the form $A \otimes I$ to random variables of the form $f\left(\omega_{1}, \omega_{2}\right)=f\left(\omega_{1}\right)$, and vice versa.

For the time being this is all the general theory that we need. We now turn to the construction of the class of models that we will consider throughout the paper.

2.3. Two-Level Systems. In this article all quantum systems will be built up (by means of tensor products) from two-level systems, i.e., quantum probability spaces with $\operatorname{dim} \mathrm{H}=2$. Any observable of a two-level system is at most a two-state random variable, which makes the theory particularly simple. Nonetheless we will find a surprisingly rich structure in the resulting models. Moreover, it turns out that such models can approximate closely the behavior of realistic physical systems; see section 10. The classical counterpart of such models, i.e., the approximation of a continuous system by a sequence of coin flips, is well known, e.g., in mathematical finance [26, 75].

We already encountered a two-level system in section 2.1. As we will use these systems so often, however, it will be useful to fix some notation and to introduce some standard vectors and operators which we will use throughout the paper.

Let $\mathscr{M}$ denote the ${ }^{*}$-algebra of all $2 \times 2$ complex matrices acting on the Hilbert space $\mathrm{H}=\mathbb{C}^{2}$. This algebra, together with suitable choices of states, will be the building block in our model of an atom in interaction with an electromagnetic field: the atom will be built on $\mathscr{M}$ (see Example 2.8), while the field will be modeled by a tensor product of $\mathscr{M}$ 's (see section 2.5).

The canonical two-level system is described by the quantum probability space $(\mathscr{M}, \rho)$, where $\rho$ is some state on $\mathscr{M}$. Implicit in this description is the choice of a standard basis (hence the name canonical) which allows us to represent linear operators by the more convenient matrices; there is no loss of generality in doing this, and it will make our life much easier. We introduce the following standard definitions:

$$
\sigma_{-}=\left(\begin{array}{cc}
0 & 0 \\
1 & 0
\end{array}\right), \quad \sigma_{+}=\sigma_{-}^{*}=\left(\begin{array}{cc}
0 & 1 \\
0 & 0
\end{array}\right), \quad \Phi=\left(\begin{array}{l}
0 \\
1
\end{array}\right) .
$$

$\Phi$ is called the vacuum vector or ground state vector for reasons which will become clear shortly. Note that $\sigma_{-} \Phi=0, \sigma_{-}^{2}=0$, and $\sigma_{-} \sigma_{+}+\sigma_{+} \sigma_{-}=I . \sigma_{+}$and $\sigma_{-}$are convenient operators, as any matrix $M \in \mathscr{M}$ can be written as a linear combination of $\sigma_{+} \sigma_{-}, \sigma_{+}, \sigma_{-}, I$. We will also sometimes use the Pauli matrix

$$
\sigma_{z}=\sigma_{+} \sigma_{-}-\sigma_{-} \sigma_{+}=\left(\begin{array}{cc}
1 & 0 \\
0 & -1
\end{array}\right)
$$

Example 2.8 (two-level atom). In this example we are going to interpret the quantum probability space $(\mathscr{M}, \rho)$ as the set of observables describing a single atom. Introduce the self-adjoint operator $H \in \mathscr{M}$ by

$$
H=\frac{\hbar \omega_{0}}{2} \sigma_{z}=\left(\begin{array}{cc}
\frac{\hbar \omega_{0}}{2} & 0 \\
0 & -\frac{\hbar \omega_{0}}{2}
\end{array}\right)
$$


where $\hbar$ is Planck's constant (a fundamental constant of nature), $\omega_{0}$ is a parameter representing a frequency (the so-called atomic frequency), and $\sigma_{z}$ is the Pauli matrix given by (2.2). As an observable, the Hamiltonian $H$ is interpreted as the energy of the atom (indeed, $\hbar \omega_{0}$ has units of energy). If an experiment is done to measure it, then from (2.3) it is clear that the outcome is a random variable taking either the value $\hbar \omega_{0} / 2$ or $-\hbar \omega_{0} / 2$ (note that $H$ is already diagonal in the given basis).

The fact that the atomic energy takes discrete values is a fundamental result in quantum mechanics to which the theory owes its name. No actual atom has only two energy levels, but in some cases atomic physics is well approximated by such a description. Other physical observables, such as the intrinsic angular momentum (spin) of an electron, are exactly described by a two-level system. Throughout this article we will use the two-level system as a prototypical example of an atom.

We have not yet discussed the state $\rho$; the state depends on the way the atom was prepared (e.g., is the atom at room temperature, has it been cooled to absolute zero, or was it perhaps excited by a laser?). A particularly interesting state, the ground state, is the one in which the energy takes its lowest possible value $-\hbar \omega_{0} / 2$ with unit probability. This situation describes an atom that has been cooled to absolute zero temperature (a good approximation for laser-cooled atoms). It is not difficult to verify that the ground state is defined by $\rho(X)=\langle\Phi, X \Phi\rangle$, where $\Phi$ is the ground state vector. In general we can choose any state for the atom, i.e., we set

$$
\rho(X)=\operatorname{Tr}[\tilde{\rho} X] \quad \text { with } \quad \tilde{\rho}=\left(\begin{array}{cc}
\rho_{11} & \rho_{12} \\
\rho_{21} & \rho_{22}
\end{array}\right), \quad X \in \mathscr{M},
$$

where $\tilde{\rho}$ is a density matrix $(\tilde{\rho} \geq 0, \operatorname{Tr} \tilde{\rho}=1)$. Then measurement of $H$ yields the outcome $-\hbar \omega_{0} / 2$ with probability $\rho_{22}$ and $\hbar \omega_{0} / 2$ with probability $\rho_{11}$. Note that the expectation value of the energy $H$ is given by $\rho(H)=\operatorname{Tr}[\tilde{\rho} H]=\frac{1}{2} \hbar \omega_{0}\left(\rho_{11}-\rho_{22}\right)$.

Apart from the energy $H$ the two-level atom may possess an electric dipole moment, described by the vector observable $\boldsymbol{\mu}$. Its three components are given by

$$
\mu_{a}=\alpha_{a} \sigma_{-}+\alpha_{a}^{*} \sigma_{+}=\left(\begin{array}{cc}
0 & \alpha_{a}^{*} \\
\alpha_{a} & 0
\end{array}\right), \quad a \in\{x, y, z\},
$$

where $\alpha_{x}, \alpha_{y}$, and $\alpha_{z}$ are complex parameters (depending on which specific atom we are considering). Note that $\mu_{a}, a \in\{x, y, z\}$, does not commute with $H$. Therefore $\mu_{a}$ and $H$ cannot be simultaneously diagonalized by the spectral theorem; that is, they cannot be represented on the same probability space. It is impossible to devise an experiment that measures both observables simultaneously. Note further that $\mu_{x, y, z}$ do not commute with each other unless $\alpha_{x, y, z}$ have the same phase, i.e., it is in general not even possible to measure all three components of $\mu$ in one realization.

2.4. The Discrete Field. Having obtained a simple model for an atom, we proceed by introducing a simple model for the electromagnetic field, e.g., light emitted from a laser. In the next section we will couple the atom and the field, which will allow us to describe measurements of light emitted from or scattered by the atom. Before we can do this, however, we must model the field in the absence of the atom.

The model we have in mind is illustrated in Figure 2.1. Imagine a laser beam which we are measuring with a photodetector. The beam propagates toward the photodetector at the speed of light, so at each time $t$ we will measure a different part of the beam. We wish to describe the measurements made by the photodetector at each time $t$. To simplify matters we will discretize time into slices. If these slices are sufficiently small (as compared to the intensity of the laser), then to good approximation 


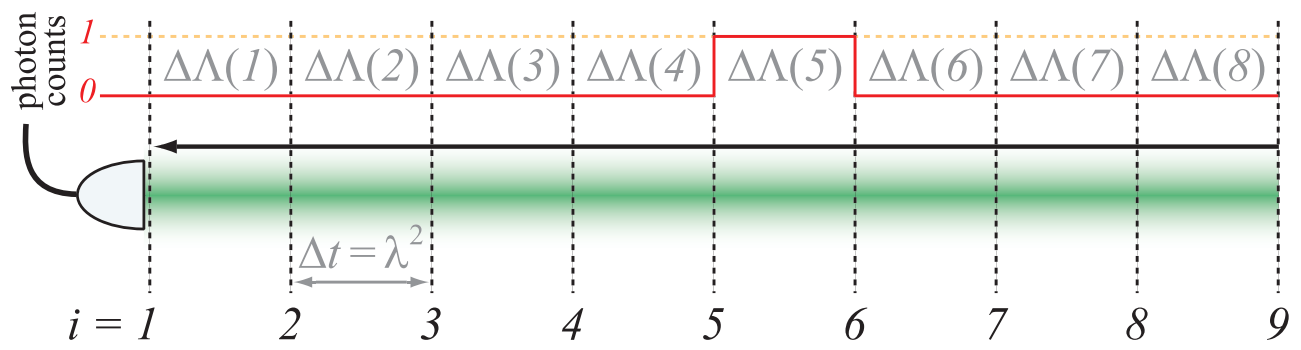

Fig. 2.I Illustration of a discretized model for the electromagnetic field. The field (e.g., a laser beam) propagates to the left until it hits a photodetector. When we discretize time with sufficiently small $\Delta t=\lambda^{2}$, then to good approximation the photodetector will detect either zero or one photon in each time step, and moreover the number of photons detected in each time interval are independent random variables. The observable $\Delta \Lambda(i)$ represents the number of photons detected in the time interval $i$. A possible sample path for the stochastic process $\iota(\Delta \Lambda(i))$ is displayed in red.

the photodetector will measure no more than one photon in each slice. Moreover, the measurement in each slice will be independent from the other slices, as we are measuring independent parts of the field at each time step. Hence we will model the photodetection of the field by a collection of independent $\{0,1\}$-valued random variables, one for each time slice, corresponding to whether a photon was (1) or was not (0) detected in each slice.

The model being a quantum mechanical model, the above description is not sufficient. The events detected by a photodetector are classical events, corresponding to an entire sample path of observations measured in a single realization, and hence are necessarily described by a commutative algebra. Other observations of the field are possible, using a different detection setup, which are incompatible with direct photodetection (we will encounter an example below). Hence we need a noncommutative model. Still, the considerations above are very suggestive; we will build our model from a collection of independent two-level systems, one for each time slice.

Fix an integer $k$ and finite time interval $[0, T], T>0$. Define $\lambda=\sqrt{T / k}$, i.e., the interval $[0, T]$ is divided into $k$ parts of equal length $\lambda^{2}$. To every time slice $1, \ldots, k$ we want to associate an independent copy of the canonical two-level system $(\mathscr{M}, \rho)$. But we have already seen how to do this: we must use tensor products, i.e., we introduce the quantum probability space

$$
\left(\mathscr{W}_{k}, \rho_{k}\right)=\left(\mathscr{M}^{\otimes k}, \rho^{\otimes k}\right)=(\underbrace{\mathscr{M} \otimes \cdots \otimes \mathscr{M}}_{k \text { times }}, \underbrace{\rho \otimes \cdots \otimes \rho}_{k \text { times }}) .
$$

For any $X \in \mathscr{M}$ and integer $1 \leq i \leq k$, we define an element $X_{i} \in \mathscr{W}_{k}$ by

$$
X_{i}=I^{\otimes(i-1)} \otimes X \otimes I^{\otimes(k-i)} .
$$

$X_{i}$ is the observable $X$ in the time slice $i$. Note the fundamental property $\left[X_{i}, Y_{j}\right]=0$ $\forall X, Y \in \mathscr{M}, i \neq j$ : measurements on different slices of the field are always compatible. For future reference, we also define the algebra $\mathscr{M}_{i}$ of observables in time slice $i$ :

$$
\mathscr{M}_{i}=\operatorname{alg}\left\{X_{i}: X \in \mathscr{M}\right\} .
$$


Discrete Noises. For $1 \leq i \leq k$ we define the standard increments

$$
\begin{aligned}
& \Delta A(i)=\lambda\left(\sigma_{-}\right)_{i}, \quad \Delta \Lambda(i)=\left(\sigma_{+} \sigma_{-}\right)_{i}, \\
& \Delta A^{*}(i)=\lambda\left(\sigma_{+}\right)_{i}, \quad \Delta t(i)=\lambda^{2} I,
\end{aligned}
$$

and consequently we introduce the discrete noise processes $A, A^{*}, \Lambda$ and the discrete time process $t$ (with the convention $A(0)=A^{*}(0)=\Lambda(0)=t(0)=0$ ) as follows:

$$
\begin{array}{ll}
A(l)=\sum_{i=1}^{l} \Delta A(i), \quad & \Lambda(l)=\sum_{i=1}^{l} \Delta \Lambda(i), \\
A^{*}(l)=\sum_{i=1}^{l} \Delta A^{*}(i), & t(l)=\sum_{i=1}^{l} \Delta t(i) .
\end{array}
$$

The particular scaling of these expressions with $\lambda$ has been selected so that the definitions make sense in the continuous limit $\lambda \rightarrow 0$; see section 10. For now, let us furnish these objects with a suitable interpretation. $t(l)=l \lambda^{2} I$ is the easiest: this observable takes the value $l \lambda^{2}$ with probability 1 . We interpret $t(l)$ as the time that has elapsed after time slice $l$. Next, let us investigate $\Lambda(l)$.

Photon Counting. For the observable $\sigma_{+} \sigma_{-} \in \mathscr{M}$, it is easily verified that the spectral theorem maps to a random variable $\iota\left(\sigma_{+} \sigma_{-}\right)$that takes the value zero or one; indeed, the matrix corresponding to $\sigma_{+} \sigma_{-}$(calculated using (2.1)) is given by

$$
\sigma_{+} \sigma_{-}=\left(\begin{array}{ll}
1 & 0 \\
0 & 0
\end{array}\right)
$$

which is already in diagonal form. $\Delta \Lambda(i), i=1, \ldots, k$, is a set of independent observables taking the value zero or one. We interpret $\Delta \Lambda(i)$ as the number of photons observed by a photodetector in the time slice $i$ (as illustrated in Figure 2.1).

The probability of observing a photon in the time slice $i$ depends on the state $\rho$; in the presence of a light source (a laser or a light bulb) this probability will be nonzero. If we turn off the light source, or if there was no light source in the first place, then the probability of measuring a photon will be zero in every time slice. As we discussed in the context of a two-level atom, this situation is described by the state $\phi(X)=\langle\Phi, X \Phi\rangle$ (i.e., $\rho_{k}=\phi^{\otimes k}$ ) - it is for this reason that this state is called the vacuum state. Even in the absence of an external light source an atom can still interact with the vacuum; e.g., an excited atom can spontaneously emit a photon into the vacuum, an event which we will want to measure using our photodetector. For concreteness, in what follows we will always work with the vacuum state $\phi^{\otimes k}$.

Now recall that $\Delta \Lambda(i), i=1, \ldots, k$, all commute with each other, and hence generate a commutative ${ }^{*}$-algebra $\mathscr{C}_{k}$. The spectral theorem maps $\mathscr{C}_{k}$ to a classical space $\left(\Omega_{k}, \mathcal{F}_{k}\right)$, which is simply the probability space corresponding to $k$ independent coin flips (a "binomial model" in the terminology of [75]). The classical stochastic process $y_{l}=\iota(\Delta \Lambda(l))$ is then precisely the signal we observe coming from the photodetector. By applying the spectral theorem to the commutative subalgebras $\mathscr{C}_{l}=\operatorname{alg}\{\Delta \Lambda(i): i=1, \ldots, l\}, \mathscr{C}_{l} \subset \mathscr{C}_{l+1} \subset \cdots \subset \mathscr{C}_{k}$, we obtain an increasing family of $\sigma$-algebras $\mathcal{F}_{l} \subset \mathcal{F}_{l+1} \subset \cdots \subset \mathcal{F}_{k}$, the filtration generated by the stochastic process $y_{l}$. As in the classical theory, the quantum filtration $\left\{\mathscr{C}_{l}\right\}$ allows us to keep track of what information is available based on observations up to and including time slice $l$.

It remains to consider $\Lambda(l) \in \mathscr{C}_{l}$; it is evident from the definition that this observable represents the number of photons observed up to and including time slice $l$. 
Hence the number process $\{\Lambda(l)\}_{l=1, \ldots, k}$, which is clearly commutative as $\Lambda(l) \in \mathscr{C}_{k}$ $\forall l$, maps to a classical counting process $\iota(\Lambda(l))$. All of this is not so interesting when we are working under the measure $\mathbf{P}_{k}$ induced by the vacuum $\phi^{\otimes k}$ on $\left(\Omega_{k}, \mathcal{F}_{k}\right)$ : under this measure any sample path of $\iota(\Lambda(l))$ that is not identically zero has zero probability. Once we have introduced the interaction with an atom, however, the number process will allow us to count the number of photons emitted by the atom.

Homodyne Detection. The processes $A(l)$ and $A^{*}(l)$ are not self-adjoint, but $X_{\varphi}(l)=e^{i \varphi} A(l)+e^{-i \varphi} A^{*}(l)$ is an observable for any phase $\varphi \in[0, \pi)$. Moreover, $\left\{X_{\varphi}(l)\right\}_{l=1, \ldots, k}$ is a commutative process for fixed $\varphi$ : to see this, note that $\Delta X_{\varphi}(l)=$ $X_{\varphi}(l)-X_{\varphi}(l-1)=\lambda\left(e^{i \varphi} \sigma_{-}+e^{-i \varphi} \sigma_{+}\right)_{l}$ commute with each other for different $l$, so the same must hold for $X_{\varphi}(l)$. Hence, as above, we can use the spectral theorem to $\operatorname{map} \mathscr{C}_{k}^{\varphi}=\operatorname{alg}\left\{X_{\varphi}(l): l=1, \ldots, k\right\}$ to a classical space $\left(\Omega_{k}^{\varphi}, \mathcal{F}_{k}^{\varphi}\right)$, and similarly we obtain the filtration $\left\{\mathcal{F}_{l}^{\varphi}\right\}_{l=1, \ldots, k}$. Beware, however: $\left\{X_{\varphi}\right\}$ does not commute with $\{\Lambda\}$, nor does it commute with $\left\{X_{\varphi^{\prime}}\right\}$ for $\varphi^{\prime} \neq \varphi$. To observe each of these processes we need fundamentally different detectors, so that in any given realization at most one of these processes is available to us.

An optical detector that measures one of the processes $X_{\varphi}(l)$ is known as a homodyne detection setup, a term borrowed from radio engineering. What is measured in such a setup is usually interpreted by considering the electromagnetic field to be a wave at a certain frequency, as in classical electromagnetism. The homodyne detector then measures the component of this wave that is in phase with a certain reference wave, known as the local oscillator, and the parameter $\varphi$ determines the phase of the local oscillator with respect to an external reference. The interpretation of the light in terms of waves (homodyne) or particles (photodetection) are not at odds, because the corresponding measurements $\left\{X_{\varphi}\right\}$ and $\{\Lambda\}$ do not commute. We will not dwell further on the physical interpretation of these detection schemes; suffice it to say that both homodyne detection and photodetection are extremely common techniques, either of which might be more convenient in any particular situation. The interested reader is referred to the quantum optics literature [81] for further information (see also [7] for a more mathematical perspective).

For concreteness we will always consider the case $\varphi=0$ whenever we discuss homodyne detection in what follows, i.e., we will consider the observation process $X(l)=A(l)+A^{*}(l)$. Let us investigate this process a little more closely. First, consider the two-level system observable $\sigma_{+}+\sigma_{-}$:

$$
\sigma_{+}+\sigma_{-}=\left(\begin{array}{ll}
0 & 1 \\
1 & 0
\end{array}\right)=\left(\begin{array}{cc}
\frac{1}{2} & \frac{1}{2} \\
\frac{1}{2} & \frac{1}{2}
\end{array}\right)-\left(\begin{array}{cc}
\frac{1}{2} & -\frac{1}{2} \\
-\frac{1}{2} & \frac{1}{2}
\end{array}\right)=P_{1}-P_{-1} .
$$

$P_{ \pm 1}$ are projections, so we can see that $\sigma_{+}+\sigma_{-}$has eigenvalues \pm 1 . Moreover, note that $\left\langle\Phi, P_{ \pm 1} \Phi\right\rangle=1 / 2$. Hence the spectral theorem evidently maps $\sigma_{+}+\sigma_{-}$to a random variable taking the values \pm 1 each with probability $1 / 2$ in the vacuum. It follows immediately that $\iota(\Delta X(l)), l=1, \ldots, k$, are independent random variables taking values $\pm \lambda$ with equal probability. Unlike in the case of photodetection, the homodyne detector gives a noisy signal even in the vacuum, a manifestation of vacuum fluctuations, as this is called in quantum optics. The process $\iota(X(l))=\sum_{i=1}^{l} \iota(\Delta X(i))$, the integrated photocurrent, is evidently a symmetric random walk on the grid $\lambda \mathbb{Z}$. It is not difficult to see that $\iota\left(X\left(\left\lfloor t / \lambda^{2}\right\rfloor\right)\right)$ converges in law to a Wiener process as $\lambda \rightarrow 0$ (recall $t(l)=l \lambda^{2}$, so $\left.l(t) \sim\left\lfloor t / \lambda^{2}\right\rfloor\right)$; see section 10 .

Remark 2.9. The reader might wonder at this point why we have chosen the notation $\iota(\Delta \Lambda(l))$ or $\iota(\Delta X(l))$ for the observations - why not dispose of the $\Delta$ 's? The 


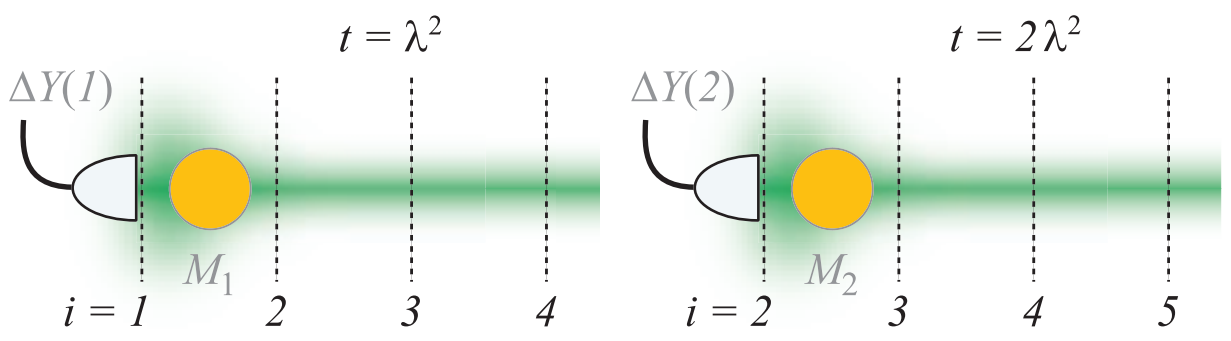

Fig. 2.2 Illustration of the discretized atom-field interaction. In each time step $i$, the atom and the field at time slice $i$ interact through a unitary transformation $M_{i}$. The time slice of the field is subsequently measured (using a photodetector or homodyne detection), which gives rise to the observation $\Delta Y(i)$. The field then propagates to the left by one time slice, and the procedure repeats.

reason is that neither $\Delta X\left(\left\lfloor t / \lambda^{2}\right\rfloor\right)$ nor $\Delta \Lambda\left(\left\lfloor t / \lambda^{2}\right\rfloor\right)$ have well-defined limits as $\lambda \rightarrow 0$; for example, $\iota\left(\Delta X\left(\left\lfloor t / \lambda^{2}\right\rfloor\right)\right)$ would have to converge to white noise, which is not a mathematically well-defined object (at least in the sense of stochastic processes). The Wiener process, on the other hand, has a rigorous definition. We use the convention that processes such as $X\left(\left\lfloor t / \lambda^{2}\right\rfloor\right)$ have well-defined limits, whereas $\Delta X\left(\left\lfloor t / \lambda^{2}\right\rfloor\right)$ will "converge" to $d X_{t}$, which is meaningful only under the integral sign.

Remark 2.10. It should be emphasized that the model we have introduced here is rather crude; certainly it was not derived from physical principles! Its main physical justification is that it converges in the limit $\lambda \rightarrow 0$ to a model which is obtained from physical principles, so that for small $\lambda$ we can consider it to be a good approximationsee the references in section 10 . The same disclaimer holds for what we will discuss in the remainder of section 2 .

2.5. Repeated Interaction. Now that we have descriptions of an atom and of the electromagnetic field, it is time to describe how these objects interact with each other. The atom can emit photons into the vacuum, which we subsequently detect using a photodetector; alternatively, using a homodyne detection setup we can measure how the presence of the atom perturbs the usual vacuum fluctuations. The remainder of the article is centered around the following theme: how can we put such measurements to good use? The development of filtering theory allows us to estimate atomic observables based on the measurements in the field, and we can subsequently use this information to control the atom by means of feedback control.

The way the interaction works is illustrated in Figure 2.2. As before, the field propagates toward the detector, which measures sequentially time slices of the field. Now, however, we place the atom right in front of the detector, so that the current time slice interacts with the atom prior to being detected. This sort of model is called a repeated interaction: the atom is fixed, and interacts identically with each time slice of the field exactly once before it is detected. Before we can describe the repeated interaction in detail, we first have to describe how a single interaction is modeled in quantum mechanics. Though this article is mostly about quantum probability, we need a little bit of quantum dynamics in order to model physical systems.

Interactions in Quantum Mechanics. Suppose we have two independent twolevel atoms, i.e., we are working on the quantum probability space $(\mathscr{M} \otimes \mathscr{M}, \rho \otimes \rho)$. 
Then, for example, the observable $\mu_{x}^{1}=\mu_{x} \otimes I$ corresponds to the electric dipole moment of the first atom, and is independent from any observable $I \otimes X$ of the second atom. If the two atoms subsequently interact, however, then the dipole moment of the first atom would likely change; i.e., the dipole moment after the interaction is described by a different observable $\mu_{x}^{1, \text { after }}$ than before the interaction, and will likely be correlated with some observable of the second atom $(I \otimes X)^{\text {after }}$.

In quantum mechanics, every interaction is described by a unitary operator. In the previous example, the interaction between the two atoms corresponds to a suitably chosen unitary map $U: \mathbb{C}^{2} \otimes \mathbb{C}^{2} \rightarrow \mathbb{C}^{2} \otimes \mathbb{C}^{2}$. This means that any observable $X \in \mathscr{M} \otimes \mathscr{M}$ transforms to $U^{*} X U \in \mathscr{M} \otimes \mathscr{M}$ after the two atoms have interacted. In particular, $\mu_{x}^{1, \text { after }}=U^{*} \mu_{x}^{1} U$ will generally be different from $\mu_{x}^{1}$, and is likely to be correlated with $U^{*}(I \otimes X) U$ for some $X$. Note that unitary rotation does not change the spectrum of an observable; i.e., $\iota\left(\mu_{x}^{1}\right)$ and $\iota\left(\mu_{x}^{1, \text { after }}\right)$ take the same values, but they are defined on different probability spaces with different probability measures.

Recall that any unitary matrix $U$ can be written as $U=e^{-i B}$ for some self-adjoint matrix $B$. Hence we can express any unitary transformation of $\mathscr{M} \otimes \mathscr{M}$ by

$$
U=\exp \left(-i\left\{L_{1} \otimes \sigma_{+} \sigma_{-}+L_{2} \otimes \sigma_{+}+L_{2}^{*} \otimes \sigma_{-}+L_{3} \otimes I\right\}\right),
$$

where $L_{1,2,3} \in \mathscr{M}$ and $L_{1}, L_{3}$ are self-adjoint.

Remark 2.11. We have described interactions by changing the observables corresponding to a particular physical quantity. This is how dynamics is usually represented in probability theory. For example, the classical discrete time Markov chain $X_{n}=f\left(X_{n-1}, \xi_{n}\right)$, where $\xi_{n}$ is i.i.d. noise, is precisely of this form: the random variables $X_{n}$ and $X_{n-1}$ represent the same physical quantity $X$, but the interaction with the noise $\xi$ means we have to represent $X$ by different random variables $X_{n}$ at different times. In quantum mechanics, this is called the Heisenberg picture. In the Schrödinger picture, any physical quantity is always represented by the same observable, but the underlying state is changed by the interaction. The two pictures are in some sense dual to each other, as is discussed in any quantum mechanics textbook (e.g., [67]). However, the Schrödinger picture is somewhat unnatural if one wants to work with (quantum) stochastic processes. In this article we will always work in the Heisenberg picture.

Atom-Field Interaction. We now consider the case that a two-level atom is coupled to the vacuum electromagnetic field, that is, we work on the quantum probability space $\left(\mathscr{M} \otimes \mathscr{W}_{k}, \mathbb{P}\right)$ with $\mathbb{P}=\rho \otimes \phi^{\otimes k}$. The subspace $(\mathscr{M}, \rho) \subset\left(\mathscr{M} \otimes \mathscr{W}_{k}, \mathbb{P}\right)$ is known as the initial system ( $\rho$ is called the initial state), because observables of the form $X \otimes I$ in $\mathscr{M} \otimes \mathscr{W}_{k}$ represent the atom's physical quantities at the initial time $l=0$, i.e., before the atom has interacted with the field. The interaction requires us to modify these observables at each time $l>0$, as we will now describe.

Let us begin by considering what happens to the system after one time step. At this time $(l=1)$ the atom has interacted only with the first time slice of the field, i.e., any atomic observable $X \otimes I$ at time $l=0$ will evolve to $j_{1}(X)=M(1)^{*}(X \otimes I) M(1)$, where $M(1)$ is the unitary interaction of the form

$$
M(1)=e^{-i\left\{L_{1} \Delta \Lambda(1)+L_{2} \Delta A^{*}(1)+L_{2}^{*} \Delta A(1)+L_{3} \otimes \Delta t(1)\right\}} .
$$

In the next time step, we want the atom to interact in an identical fashion with the second time slice, so we will define $j_{2}(X)=M(2)^{*} j_{1}(X) M(2)$ with

$$
M(2)=e^{-i\left\{j_{1}\left(L_{1}\right) \Delta \Lambda(2)+j_{1}\left(L_{2}\right) \Delta A^{*}(2)+j_{1}\left(L_{2}^{*}\right) \Delta A(2)+j_{1}\left(L_{3}\right) \Delta t(2)\right\}} .
$$


Note that not only do we need to change the time slice that we are interacting with, but we also need to propagate the atomic observables that define the interaction.

Remark 2.12. Once again it is useful to bear in mind the example of the classical Markov chain $X_{n}=f\left(X_{n-1}, \xi_{n}\right)$. Suppose $X_{n}$ takes values in $\mathbb{R}^{k}$; if we are looking, e.g., at the first component $X_{n}^{1}$, then $X_{n}^{1}=f\left(X_{n-1}^{1}, X_{n-1}^{2}, \ldots, X_{n-1}^{k}, \xi_{n}\right)$. Hence the transformation $f\left(\cdot, X_{n-1}^{2}, \ldots, X_{n-1}^{k}, \xi_{n}\right)$ of $X_{n-1}^{1}$ depends not only on the noise "time slice" $\xi_{n}$, but also on other "observables" of the system $X_{n-1}^{2}, \ldots, X_{n-1}^{k}$ which must be evaluated at time $n-1$. Similarly our quantum mechanical interaction $M$ depends at each time step $l$ on the $l$ th time slice in the field as well as on the atomic observables in the previous time step $l-1$.

Proceeding in a similar fashion, we find that any atomic observable $X \in \mathscr{M}$ has evolved to the observable $j_{l}(X)=U(l)^{*}(X \otimes I) U(l) \in \mathscr{M} \otimes \mathscr{W}_{k}$ at time $l$, where

$$
U(l)=\vec{\prod}_{i=1}^{l} M(i)=M(1) M(2) \cdots M(l), \quad U(0)=I,
$$

is a repeated interaction with the one time step interaction

$$
M(l)=e^{-i\left\{j_{l-1}\left(L_{1}\right) \Delta \Lambda(l)+j_{l-1}\left(L_{2}\right) \Delta A^{*}(l)+j_{l-1}\left(L_{2}^{*}\right) \Delta A(l)+j_{l-1}\left(L_{3}\right) \Delta t(l)\right\}} .
$$

The map $j_{l}(\cdot)$ defines the time evolution or flow of the atomic observables. The connection with dynamical systems theory can be made explicit: if we define $U(i, l)=$ $M(i+1) M(i+2) \cdots M(l), i<l$, and $J_{i, l}(X)=U(i, l)^{*} X U(i, l)$ for $X \in \mathscr{M} \otimes \mathscr{W}_{k}$, then $J_{i, l}(\cdot)$ is a two-parameter group of transformations of the algebra $\mathscr{M} \otimes \mathscr{W}_{k}$ (i.e., for $i<l<r, J_{l, r}\left(J_{i, l}(\cdot)\right)=J_{i, r}(\cdot), J_{i, r}\left(J_{i, l}^{-1}(\cdot)\right)=J_{l, r}(\cdot)$, etc.); thus we have truly defined a discrete time dynamical system in the dynamical systems sense. We will not need this in what follows, however.

There is a different representation of the repeated interaction unitary $U(l)$ which is often more convenient. Introduce the unitaries

$$
M_{l}=e^{-i\left\{L_{1} \Delta \Lambda(l)+L_{2} \Delta A^{*}(l)+L_{2}^{*} \Delta A(l)+L_{3} \Delta t(l)\right\}},
$$

which, in contrast to $M(l)$, depend on the initial observables $L_{1,2,3}$ rather than the time-evolved observable $j_{l-1}\left(L_{1,2,3}\right)$. From $j_{l-1}\left(L_{1,2,3}\right)=U(l-1)^{*}\left(L_{1,2,3} \otimes I\right) U(l-1)$, it follows immediately that $M(l)=U(l-1)^{*} M_{l} U(l-1)$. But then

$$
U(l)=U(l-1) M(l)=U(l-1) U(l-1)^{*} M_{l} U(l-1)=M_{l} U(l-1),
$$

so we obtain the expression

$$
U(l)=\overleftarrow{\prod}_{i=1}^{l} M_{i}=M_{l} M_{l-1} \cdots M_{2} M_{1}
$$

Hence we can use the $M_{l}$ 's rather than $M(l)$ 's if we reverse the time ordering in the definition of $U(l)$. This is often convenient because $M_{l}$ is a function only of the initial system and of the $l$ th time slice, whereas $M(l)$ depends on the entire history of the field up to time slice $l$.

The choice of $L_{1,2,3} \in \mathscr{M}$ determines the nature of the physical interaction between the atom and the field. For different atoms or for different experimental scenarios this interaction can take very different forms; nonetheless the theory that we are about to develop can be set up in quite a general way for a large class of interactions. In section 2.6 we will introduce two specific examples of physical interactions which we will use throughout to illustrate the general theory. 
Observations. Up to this point we have concentrated on the time evolution $j_{l}(X)$ of an atomic observable $X \otimes I$; however, the observables corresponding to time slices of the field are also modified by the interaction with the atom in a completely analogous way. Recall, for example, that before the interaction the number of photons in time slice $l$ is represented by the observable $I \otimes \Delta \Lambda(l)$. After the interaction this quantity is represented by $\Delta Y^{\Lambda}(l)=U(l)^{*}(I \otimes \Delta \Lambda(l)) U(l)$, so that $\iota\left(\Delta Y^{\Lambda}(l)\right)$ is precisely what we observe at time $l$ if a photodetector is used to measure the field. Similarly, homodyne detection measures $\Delta Y^{X}(l)=U(l)^{*}\left(I \otimes \Delta A(l)+I \otimes \Delta A^{*}(l)\right) U(l)$ at time l. We will use the generic notation $\Delta Y(l)=U(l)^{*} \Delta Z(l) U(l)$, where $\Delta Y=\Delta Y^{\Lambda}$ if $\Delta Z=I \otimes \Delta \Lambda$ and $\Delta Y=\Delta Y^{X}$ if $\Delta Z=I \otimes\left(\Delta A+\Delta A^{*}\right)$.

The first question we should ask ourselves is, does this procedure make sense? After all, in the laboratory we measure classical stochastic processes, so that in order for our theory to be consistent the random variables $\iota(\Delta Y(l)), l=1, \ldots, k$, must define a classical stochastic process on some fixed probability space $\left(\Omega_{k}, \mathcal{F}_{k}, \mathbf{P}_{k}\right)$-in other words, $\mathscr{Y}_{k}=\operatorname{alg}\{\Delta Y(l): l=1, \ldots, k\}$ must be a commutative algebra. Let us verify that this is indeed the case. The basic insight we need is the following observation: $\left[M_{l}, \Delta Z(i)\right]=0$ for $l>i$ (this is easily verified by inspection), so that for $j>l$,

$$
\begin{aligned}
U(j)^{*} \Delta Z(l) U(j)=\left(\vec{\prod}_{i=1}^{j} M_{i}^{*}\right) \Delta Z(l)\left(\overleftarrow{\prod}_{i=1}^{j} M_{i}\right) \\
=\left(\vec{\prod}_{i=1}^{l} M_{i}^{*}\right) \Delta Z(l)\left(\overleftarrow{\prod}_{i=1}^{l} M_{i}\right)=U(l)^{*} \Delta Z(l) U(l)
\end{aligned}
$$

This is not unexpected: it is simply a statement of the fact that the $l$ th time slice of the field interacts exactly once with the atom, viz., at the $l$ th time step (note that $U(j)^{*} \Delta Z(l) U(j)=\Delta Z(l)$ for $\left.j<l\right)$, for any $l$. But then for $j>l$,

$$
\begin{aligned}
& {[\Delta Y(l), \Delta Y(j)]=\left[U(l)^{*} \Delta Z(l) U(l), U(j)^{*} \Delta Z(j) U(j)\right]} \\
& \quad=\left[U(j)^{*} \Delta Z(l) U(j), U(j)^{*} \Delta Z(j) U(j)\right]=U(j)^{*}[\Delta Z(l), \Delta Z(j)] U(j)=0,
\end{aligned}
$$

so that clearly $\mathscr{Y}_{k}$ is commutative. This self-nondemolition property guarantees that the observations can indeed be interpreted as a classical stochastic process through the spectral theorem, a crucial requirement for the physical interpretation of the theory.

Previously we introduced the filtration $\mathscr{C}_{l}=\operatorname{alg}\{\Delta Z(i): i=1, \ldots, l\}, l=$ $1, \ldots, k$, corresponding to the photodetection or homodyne detection of the field in the absence of interaction with the atom. Similarly we now define the filtration $\mathscr{Y}_{l}=\operatorname{alg}\{\Delta Y(i): i=1, \ldots, l\}, l=1, \ldots, k$, which represents the information contained in observations of the field (after it has interacted with the atom) up to the $l$ th time step. Applying the spectral theorem to $\mathscr{Y}_{k}$, we obtain a classical probability space with the corresponding observation process $\Delta y_{l}=\iota(\Delta Y(l))$ and filtration $\iota\left(\mathscr{Y}_{l}\right)=\ell^{\infty}\left(\mathcal{Y}_{l}\right) \quad$ (note that we then have $\left.\mathcal{Y}_{l}=\sigma\left\{\Delta y_{1}, \ldots, \Delta y_{l}\right\}\right)$. The following fact will be useful later on:

$$
\mathscr{Y}_{l}=U(j)^{*} \mathscr{C}_{l} U(j)=\left\{U(j)^{*} X U(j): X \in \mathscr{C}_{l}\right\} \quad \forall j \geq l .
$$

The proof is identical to the proof of the self-nondemolition property.

We conclude this section with the demonstration of an important property of the models under investigation, the nondemolition property, to which we have already 
alluded in section 2.1. Choose any $X \in \mathscr{M}$. Then for any $l \leq i$,

$$
\begin{aligned}
& {\left[\Delta Y(l), j_{i}(X)\right]=\left[U(l)^{*} \Delta Z(l) U(l), U(i)^{*}(X \otimes I) U(i)\right]} \\
& \quad=\left[U(i)^{*} \Delta Z(l) U(i), U(i)^{*}(X \otimes I) U(i)\right]=U(i)^{*}[\Delta Z(l), X \otimes I] U(i)=0 .
\end{aligned}
$$

Evidently any atomic observable in time step $i$ commutes with the entire history of observations up to that time. Thus in principle we could decide at any time to stop observing the field and we would still be able to measure any atomic observable: the joint distribution of $\Delta Y(1), \ldots, \Delta Y(l)$ and $j_{l}(X)$ is defined for any self-adjoint $X \in \mathscr{M}$ as $\operatorname{alg}\left\{\Delta Y(1), \ldots, \Delta Y(l), j_{l}(X)\right\}$ is a commutative ${ }^{*}$-algebra, despite the fact that $j_{l}\left(X_{1}\right)$ and $j_{l}\left(X_{2}\right)$ need not commute for $X_{1} \neq X_{2}$. This enables us to meaningfully define the conditional expectation of $j_{l}(X)$ with respect to the observations $\Delta Y(i), i=$ $1, \ldots, l$, for any $X \in \mathscr{M}$, which we will do in section 3. The nondemolition property provides us with a sensible way of estimating a whole set of observables, despite the fact that they do not commute with each other, as every observable in this set separately commutes with the observation history on which we are basing the estimate. If the latter were not the case, the estimates would have no physical relevance; one would not be able to devise an experiment, even in principle, which could verify the predictions of such an estimator. Similarly, it would be meaningless to try to control atomic observables which do not commute with the observations on which we have based our feedback control law. The nondemolition property avoids such problems and is thus of fundamental importance for quantum filtering and feedback control.

2.6. Examples. The interaction matrices $L_{1,2,3}$ determine the nature of the physical interaction between the atom and the field. Though we will set up the theory in the following sections for arbitrary $L_{1,2,3}$, we will repeatedly demonstrate the theory using the following two examples. The examples illustrate two common experimental scenarios - spontaneous emission and dispersive interaction - and we will be able to study these examples explicitly through numerical simulations.

Spontaneous Emission. The spontaneous emission scenario is obtained when an atom sits undisturbed in the vacuum at zero temperature. If the energy of the atom is minimal, $\mathbb{P}(H)=-\hbar \omega_{0} / 2$, then it will remain this way for all time. Otherwise, the energy of the atom decays to its minimal value. A photodetector measuring the field would see exactly one emitted photon at some randomly distributed time: hence the name "spontaneous emission." We will reproduce this behavior through numerical simulations once we have introduced the corresponding filtering equations.

The spontaneous emission model is characterized by the interaction matrices

$$
L_{1}=L_{3}=0, \quad L_{2}=i \sqrt{2 \kappa} \sigma_{-},
$$

where $\kappa$ is the spontaneous emission rate. For simplicity, we will always set $2 \kappa=1$. This is a discretized version of the well-known Wigner-Weisskopf model.

Remark 2.13. In principle, we could already calculate the possible observation sample paths and their probabilities at this point. After all, we would only need to simultaneously diagonalize the matrices $\Delta Y(i), i=1, \ldots, k$, obtained using this explicit choice for $L_{1,2,3}$. We will have a much more efficient way of calculating such sample paths, however, once we have introduced the filtering equations: these will allow us to simulate typical sample paths using a Monte Carlo method. Hence we postpone numerical investigation of our examples until then.

Copyright $@$ by SIAM. Unauthorized reproduction of this article is prohibited. 
Dispersive Interaction. In the spontaneous emission scenario, the atom radiates its excess energy by emitting a photon with energy $\hbar \omega_{0}$ (i.e., with frequency $\omega_{0}$ ) this is precisely the difference between the two values that the energy $H$ can take. It is possible to suppress the spontaneous emission significantly by "discouraging" the atom from emitting photons with frequency $\omega_{0}$; for example, we can place the atom in an optical cavity whose resonant frequency is far detuned from $\omega_{0}$. In this dispersive regime, the (effective) interaction between the atom and the field is rather different in nature. An atom which has energy $+\hbar \omega_{0} / 2$ will shift the phase of the output light by a small amount in one direction, while an atom with energy $-\hbar \omega_{0} / 2$ will shift the phase by the same amount in the other direction. Such phase shifts can be observed using a homodyne detection setup, and the resulting photocurrent thus carries information on the energy of the atom.

The dispersive interaction model is characterized by the interaction matrices

$$
L_{1}=L_{3}=0, \quad L_{2}=i \sqrt{g} \sigma_{z},
$$

where $g$ is the interaction strength. For simplicity, we will always set $g=1$.

3. Conditional Expectations and the Filtering Problem. Within the context of the repeated interaction models introduced in the previous section, we have established that the observation history up to time step $l$ is compatible with any atomic observable at time step $l$. In this section we will show how to estimate atomic observables based on the observation history. Because only commuting observables are involved, the corresponding theory can simply be "lifted" from classical probability theory using the spectral theorem. This is precisely what we will do.

3.I. A Brief Reminder. We begin by briefly recalling how classical conditioning works. Let $(\Omega, \mathcal{F}, \mathbf{P})$ be a probability space and let $P, Q \in \mathcal{F}$ be a pair of events. The conditional probability of $P$ given $Q$ is given by

$$
\mathbf{P}(P \mid Q)=\frac{\mathbf{P}(P \cap Q)}{\mathbf{P}(Q)},
$$

provided $\mathbf{P}(Q) \neq 0$. One could roughly interpret this quantity as follows: generate a large number of samples distributed according to the measure $\mathbf{P}$, but discard those samples for which the event $Q$ is false. Then $\mathbf{P}(P \mid Q)$ is the fraction of the remaining samples for which the event $P$ is true.

Now suppose $f: \Omega \rightarrow\left\{f_{1}, \ldots, f_{m}\right\}$ and $g: \Omega \rightarrow\left\{g_{1}, \ldots, g_{n}\right\}$ are measurable random variables that take a finite number of values. The conditional expectation of $f$ given $g$ is the random variable defined by

$$
\mathbf{E}_{\mathbf{P}}(f \mid g)(\omega)=\sum_{j=1}^{n} \chi_{g^{-1}\left(g_{j}\right)}(\omega) \sum_{i=1}^{m} f_{i} \mathbf{P}\left(f^{-1}\left(f_{i}\right) \mid g^{-1}\left(g_{j}\right)\right), \quad \omega \in \Omega .
$$

In fact, we can consider $\mathbf{E}_{\mathbf{P}}(f \mid g)(\omega)$ to be a function of $g$ :

$$
\mathbf{E}_{\mathbf{P}}(f \mid g)(\omega)=F(g(\omega)), \quad F: g_{j} \mapsto \sum_{i=1}^{m} f_{i} \mathbf{P}\left(f^{-1}\left(f_{i}\right) \mid g^{-1}\left(g_{j}\right)\right) .
$$

To interpret this quantity, again we generate a large number of samples but now we divide these up into disjoint subsets corresponding to the value taken by $g$. Now average $f$ over each of these subsets. Then $F\left(g_{j}\right)$ is the average of $f$ over the subset of samples on which $g$ takes the value $g_{j}$, so that $\mathbf{E}_{\mathbf{P}}(f \mid g)=F(g)$ is simply the 
Table 3.I Elementary properties of the conditional expectation in classical (and quantum) probability; see, e.g., [83]. Equalities are always in the sense of versions, e.g., $\mathbf{E}_{\mathbf{P}}\left(f_{1} \mid \mathcal{G}\right)+$ $\mathbf{E}_{\mathbf{P}}\left(f_{2} \mid \mathcal{G}\right)=\mathbf{E}_{\mathbf{P}}\left(f_{1}+f_{2} \mid \mathcal{G}\right)$ means that any version of $\mathbf{E}_{\mathbf{P}}\left(f_{1} \mid \mathcal{G}\right)+\mathbf{E}_{\mathbf{P}}\left(f_{2} \mid \mathcal{G}\right)$ is a version of $\mathbf{E}_{\mathbf{P}}\left(f_{1}+f_{2} \mid \mathcal{G}\right)$.

\begin{tabular}{|l|l|}
\hline Property & Description \\
\hline Linearity & $\alpha \mathbf{E}_{\mathbf{P}}\left(f_{1} \mid \mathcal{G}\right)+\beta \mathbf{E}_{\mathbf{P}}\left(f_{2} \mid \mathcal{G}\right)=\mathbf{E}_{\mathbf{P}}\left(\alpha f_{1}+\beta f_{2} \mid \mathcal{G}\right)$. \\
Positivity & If $f \geq 0$, then $\mathbf{E}_{\mathbf{P}}(f \mid \mathcal{G}) \geq 0$ a.s. \\
Invariance of expectation & $\mathbf{E}_{\mathbf{P}}\left(\mathbf{E}_{\mathbf{P}}(f \mid \mathcal{G})\right)=\mathbf{E}_{\mathbf{P}}(f)$. \\
Module property & If $g$ is $\mathcal{G}$-measurable, $\mathbf{E}_{\mathbf{P}}(f g \mid \mathcal{G})=g \mathbf{E}_{\mathbf{P}}(f \mid \mathcal{G})$ a.s. \\
& In particular, $\mathbf{E}_{\mathbf{P}}(g \mid \mathcal{G})=g \mathbf{E}_{\mathbf{P}}(1 \mid \mathcal{G})=g$ a.s. \\
Tower property & If $\mathcal{H} \subset \mathcal{G}, \mathbf{E}_{\mathbf{P}}\left(\mathbf{E}_{\mathbf{P}}(f \mid \mathcal{G}) \mid \mathcal{H}\right)=\mathbf{E}_{\mathbf{P}}(f \mid \mathcal{H})$. \\
Independence & If $\sigma\{f, \mathcal{H}\}$ is independent of $\mathcal{G}, \mathbf{E}_{\mathbf{P}}(f \mid \sigma\{\mathcal{H}, \mathcal{G}\})=\mathbf{E}_{\mathbf{P}}(f \mid \mathcal{H})$ a.s. \\
\hline
\end{tabular}

expectation of $f$ given that we know $g$. Note that we did not define the quantity $\mathbf{P}(P \mid Q)$ for the case $\mathbf{P}(Q)=0$; hence the expressions above for $\mathbf{P}(P \mid Q)$ are properly defined everywhere on $\Omega$ except on $\Omega^{0}=\bigcup_{j: \mathbf{P}\left(g^{-1}\left(g_{j}\right)\right)=0} g^{-1}\left(g_{j}\right) \in \mathcal{F}$. We allow $\mathbf{E}_{\mathbf{P}}(f \mid g)$ to take arbitrary values on $\Omega^{0}$ and call any such random variable a version of the conditional expectation of $f$ with respect to $g$. There is no loss in doing this: all versions of the conditional expectation coincide with unit probability.

The use of these expressions is rather limited, as they do not extend beyond random variables $g$ that take a finite number of values. One of Kolmogorov's fundamental insights in the axiomatization of probability theory was his abstract definition of the conditional expectation, which works in the most general settings. In this article we only use finite state random variables, but even so we find it significantly easier to use the abstract definition than these clumsy explicit expressions. Let us thus briefly recall the general setting, as can be found in any probability textbook (e.g., [83]).

Let $\mathcal{G} \subset \mathcal{F}$ be a $\sigma$-algebra on which we want to condition; i.e., these are the events which we know to be true or false due to our observations. Let $f$ be an $\mathcal{F}$-measurable random variable. Then any $\mathcal{G}$-measurable random variable $\hat{f}$ that satisfies

$$
\mathbf{E}_{\mathbf{P}}(\hat{f} g)=\mathbf{E}_{\mathbf{P}}(f g) \quad \forall \mathcal{G} \text {-measurable (bounded) } g
$$

is a version of the conditional expectation $\hat{f}=\mathbf{E}_{\mathbf{P}}(f \mid \mathcal{G})$. The conditional expectation is by construction a function of what we have observed (it is $\mathcal{G}$-measurable), as it should be. It is easily verified that the explicit expression (3.1) satisfies the abstract definition with $\mathcal{G}=\sigma\{g\}$. In fact, in the case where $\Omega$ takes a finite number of values we can always express $\mathbf{E}_{\mathbf{P}}(f \mid \mathcal{G})$ as in (3.1) by constructing $g$ specifically so that it generates $\mathcal{G}$; expressing $g$ in terms of the smallest measurable partition of $\Omega$ as in the proof of Lemma 2.4, we only need to make sure that $g$ takes a different value on each set in that partition. Hence there always exists an $\hat{f}$ that satisfies the abstract definition, and it is not difficult to prove that all versions coincide with unit probability - the proof of the latter is the same as the one given below in the quantum case. Elementary properties of the conditional expectation are listed in Table 3.1.

We conclude by recalling the geometric interpretation of the conditional expectation. For simplicity, take $\Omega=\{1, \ldots, N\}$ and fix the $\sigma$-algebra $\mathcal{G} \subset \mathcal{F}$. Clearly $\ell^{\infty}(\mathcal{F})$ is a finite-dimensional linear space and $\ell^{\infty}(\mathcal{G}) \subset \ell^{\infty}(\mathcal{F})$ is a linear subspace. Moreover, $\langle f, g\rangle_{\mathbf{P}}=\mathbf{E}_{\mathbf{P}}\left(f^{*} g\right)$ is a pre-inner product: it satisfies all the conditions of the inner product, except $\langle f, f\rangle_{\mathbf{P}}=0$ iff $f=0 \mathbf{P}$-a.s. rather than iff $f(\omega)=0$ $\forall \omega \in \Omega$. Hence under $\langle\cdot, \cdot\rangle_{\mathbf{P}}, \ell^{\infty}(\mathcal{F})$ is a pre-inner product space. With this interpretation, it is evident from $(3.2)$ that $\mathbf{E}_{\mathbf{P}}(f \mid \mathcal{G})$ is simply the orthogonal projection 
of $f \in \ell^{\infty}(\mathcal{F})$ onto $\ell^{\infty}(\mathcal{G})$. It follows from the projection theorem that $\mathbf{E}_{\mathbf{P}}(f \mid \mathcal{G})$ is the a.s. unique random variable $\hat{f} \in \ell^{\infty}(\mathcal{G})$ that minimizes the mean-square error $\langle f-\hat{f}, f-\hat{f}\rangle_{\mathbf{P}}=\mathbf{E}_{\mathbf{P}}\left(|f-\hat{f}|^{2}\right)$. This gives the conditional expectation, in addition to the probabilistic interpretation above, a firm statistical interpretation as the estimator that minimizes the mean-square error.

3.2. Quantum Conditional Expectation. Let $(\mathscr{B}, \mathbb{P})$ be a commutative quantum probability space; by the spectral theorem, it is equivalent to some classical probability space $(\Omega, \mathcal{F}, \mathbf{P})$. Moreover, the ${ }^{*}$-subalgebra $\mathscr{C} \subset \mathscr{B}$ defines through the spectral theorem a $\sigma$-subalgebra $\mathcal{G} \subset \mathcal{F}$ (as $\iota(\mathscr{C})=\ell^{\infty}(\mathcal{G}) \subset \ell^{\infty}(\mathcal{F})$ ). Because the classical and quantum probability models are completely equivalent, we can simply lift the definition of the conditional expectation to the algebraic level:

$$
\mathbb{P}(X \mid \mathscr{C})=\iota^{-1}\left(\mathbf{E}_{\mathbf{P}}(\iota(X) \mid \mathcal{G})\right) \quad \forall X \in \mathscr{B} .
$$

This is nothing but a reexpression of the conditional expectation $\mathbf{E}_{\mathbf{P}}(f \mid \mathcal{G})$ in the language of *-algebras. In fact, we can go one step further and directly translate the abstract definition (3.2) into algebraic language: any $\hat{X} \in \mathscr{C}$ that satisfies $\mathbb{P}(X C)=\mathbb{P}(\hat{X} C) \forall C \in \mathscr{C}$ is a version of $\mathbb{P}(X \mid \mathscr{C})$. We emphasize again that by the spectral theorem, observables $X \in \mathscr{B}$ are just random variables and $\mathbb{P}(X \mid \mathscr{C})$ is just the ordinary conditional expectation. The spectral theorem is a powerful tool indeed!

Usually we do not start with a commutative quantum probability space. Rather, we begin with a noncommutative space $(\mathscr{A}, \mathbb{P})$ and choose a commutative subalgebra $\mathscr{C} \subset \mathscr{A}$ corresponding to the observations - keep in mind the example of the space $\left(\mathscr{M} \otimes \mathscr{W}_{k}, \rho \otimes \phi^{\otimes k}\right)$ with the observations $\mathscr{Y}_{l} \subset \mathscr{M} \otimes \mathscr{W}_{k}$ at time step $l$. As we have seen, there could be many elements $X \in \mathscr{A}$ that commute with every element in $\mathscr{C}$, but that do not commute with each other. The set $\mathscr{C}^{\prime}=\{A \in \mathscr{A}:[A, C]=0 \forall C \in \mathscr{C}\}$ is called the commutant of $\mathscr{C}$ in $\mathscr{A}$. It is easily verified that $\mathscr{C}^{\prime}$ is always a ${ }^{*}$-algebra, but generally $\mathscr{C}^{\prime}$ is not commutative. Nonetheless we can naturally extend our previous definition of $\mathbb{P}(\cdot \mid \mathscr{C})$ from the commutative algebra $\mathscr{B}$ to the case $\mathscr{B}=\mathscr{C}^{\prime}$.

Definition 3.1 (quantum conditional expectation). Let $(\mathscr{A}, \mathbb{P})$ be a quantum probability space, and let $\mathscr{C}$ be a commutative subalgebra of $\mathscr{A}$. Then the map $\mathbb{P}(\cdot \mid \mathscr{C}): \mathscr{C}^{\prime} \rightarrow \mathscr{C}$ is called a version of the conditional expectation from $\mathscr{C}^{\prime}$ onto $\mathscr{C}$ if $\mathbb{P}(\mathbb{P}(A \mid \mathscr{C}) C)=\mathbb{P}(A C) \forall A \in \mathscr{C}^{\prime}, C \in \mathscr{C}$.

How should we interpret this definition? Let $A=A^{*} \in \mathscr{C}^{\prime}$ be an observable, and suppose we are interested in $\mathbb{P}(A \mid \mathscr{C})$. The definition of $\mathbb{P}(A \mid \mathscr{C})$ only involves the operators $A$ and $C \in \mathscr{C}$, so for the purposes of evaluating $\mathbb{P}(A \mid \mathscr{C})$ we don't need the entire algebra $\mathscr{C}^{\prime}$; we might as well confine ourselves to $\mathscr{C}_{A}=\operatorname{alg}\{A, C: C \in$ $\mathscr{C}\} \subset \mathscr{C}^{\prime}$-but $\mathscr{C}_{A}$ is commutative! Hence for any $A=A^{*} \in \mathscr{C}^{\prime}$, Definition 3.1 simply reduces to the commutative definition we gave before. For $A \neq A^{*}$, we can always write $A=P+i Q$, where $P=\left(A+A^{*}\right) / 2$ and $Q=-i\left(A-A^{*}\right) / 2$ are self-adjoint, and we define $\mathbb{P}(A \mid \mathscr{C})=\mathbb{P}(P \mid \mathscr{C})+i \mathbb{P}(Q \mid \mathscr{C})$. We do this mostly for computational convenience (e.g., to ensure that the conditional expectation is linear); at the end of the day, only conditional expectations of observables have a meaningful interpretation.

By now it should not come as a surprise that the conditional expectation can only be defined on the commutant $\mathscr{C}^{\prime}$. In section 3.1 we gave an "experimental procedure" for calculating the conditional expectation of a random variable $f$ : generate a large number of samples from the joint distribution of $f$ and the observations which we are conditioning on, separate the samples into subsets corresponding to the different observations, and average $f$ within each of these subsets. But quantum mechanics tells us that it is fundamentally impossible to devise such an experiment when the 
observable $A$ which we want to condition is incompatible with the observations! The commutant $\mathscr{C}^{\prime}$ is thus precisely the right notion for a meaningful definition of the quantum conditional expectation. ${ }^{1}$

Having introduced an abstract definition, let's do a little ground work.

Lemma 3.2. $\mathbb{P}(A \mid \mathscr{C}), A \in \mathscr{C}^{\prime}$ exists and is unique with probability 1 ; i.e., any two versions $P$ and $Q$ of $\mathbb{P}(A \mid \mathscr{C})$ satisfy $\mathbb{P}\left((P-Q)^{*}(P-Q)\right)=0$.

Proof. We first introduce some notation. $\langle F, G\rangle_{\mathbb{P}}=\mathbb{P}\left(F^{*} G\right)$ defines a pre-inner product on $\mathscr{C}^{\prime}$, thus turning it into a pre-inner product space. The associated seminorm is denoted by $\|F\|_{\mathbb{P}}=\left(\langle F, F\rangle_{\mathbb{P}}\right)^{1 / 2}$.

Existence. For any self-adjoint $A=A^{*} \in \mathscr{C}^{\prime}$, define $\mathscr{C}_{A}=\operatorname{alg}\{A, C: C \in$ $\mathscr{C}\}$. The spectral theorem then provides a probability space $\left(\Omega_{A}, \mathcal{F}_{A}, \mathbf{P}_{A}\right)$ and a *isomorphism $\iota_{A}: \mathscr{C}_{A} \rightarrow \ell^{\infty}\left(\mathcal{F}_{A}\right)$, and we write $\iota_{A}(\mathscr{C})=\ell^{\infty}\left(\mathcal{G}_{A}\right)$. For every such $A$, define $\mathbb{P}(A \mid \mathscr{C})=\iota_{A}^{-1}(a)$, where $a$ is some version of $\mathbf{P}_{A}\left(\iota_{A}(A) \mid \mathcal{G}_{A}\right)$. For nonself-adjoint $A \in \mathscr{C}^{\prime}$, define $\mathbb{P}(A \mid \mathscr{C})=\mathbb{P}(P \mid \mathscr{C})+i \mathbb{P}(Q \mid \mathscr{C})$ with $P=\left(A+A^{*}\right) / 2$ and $Q=-i\left(A-A^{*}\right) / 2$. It is easily verified that the map $\mathbb{P}(\cdot \mid \mathscr{C})$ thus constructed satisfies Definition 3.1.

Uniqueness with probability 1 . Let $P$ and $Q$ be two versions of $\mathbb{P}(A \mid \mathscr{C})$. From Definition 3.1, it follows that $\langle C, P-Q\rangle_{\mathbb{P}}=0$ for all $C \in \mathscr{C}$. But $P-Q \in \mathscr{C}$, so $\langle P-Q, P-Q\rangle_{\mathbb{P}}=\|P-Q\|_{\mathbb{P}}^{2}=0$.

Let us prove that the conditional expectation is the least mean-square estimate.

Lemma 3.3. $\mathbb{P}(A \mid \mathscr{C})$ is the least mean-square estimate of $A$ given $\mathscr{C}$; i.e., for any $C \in \mathscr{C}$ we have $\|A-\mathbb{P}(A \mid \mathscr{C})\|_{\mathbb{P}} \leq\|A-C\|_{\mathbb{P}}$.

Proof. For any $C \in \mathscr{C}$ we have $\|A-C\|_{\mathbb{P}}^{2}=\|A-\mathbb{P}(A \mid \mathscr{C})+\mathbb{P}(A \mid \mathscr{C})-C\|_{\mathbb{P}}^{2}$. Now note that, by Definition 3.1, we have $\langle C, A-\mathbb{P}(A \mid \mathscr{C})\rangle_{\mathbb{P}}=\mathbb{P}\left(C^{*} A\right)-\mathbb{P}\left(C^{*} \mathbb{P}(A \mid \mathscr{C})\right)=0$ for all $C \in \mathscr{C}, A \in \mathscr{C}^{\prime}$, i.e., $A-\mathbb{P}(A \mid \mathscr{C})$ is orthogonal to $\mathscr{C}$. In particular, $A-\mathbb{P}(A \mid \mathscr{C})$ is orthogonal to $\mathbb{P}(A \mid \mathscr{C})-C \in \mathscr{C}$, and we obtain $\|A-C\|_{\mathbb{P}}^{2}=\|A-\mathbb{P}(A \mid \mathscr{C})\|_{\mathbb{P}}^{2}+$ $\|\mathbb{P}(A \mid \mathscr{C})-C\|_{\mathbb{P}}^{2}$. The result follows immediately.

The elementary properties listed in Table 3.1 and their proofs carry over directly to the quantum case. For example, let us prove linearity. It suffices to show that $Z=\alpha \mathbb{P}(A \mid \mathscr{C})+\beta \mathbb{P}(B \mid \mathscr{C})$ satisfies the definition of $\mathbb{P}(\alpha A+\beta B \mid \mathscr{C})$, i.e., $\mathbb{P}(Z C)=$ $\mathbb{P}((\alpha A+\beta B) C)$ for all $C \in \mathscr{C}$. But this is immediate from the linearity of $\mathbb{P}$ and Definition 3.1. We encourage the reader to verify the remaining properties.

In section 6 we will need to relate conditional expectations with respect to different states to each other. This is done by the following Bayes-type formula.

LEMma 3.4 (Bayes formula). Let $(\mathscr{A}, \mathbb{P})$ be a quantum probability space, and let $\mathscr{C}$ be a commutative subalgebra of $\mathscr{A}$. Let $V$ be an element in $\mathscr{C}^{\prime}$ such that $V^{*} V>0$ and $\mathbb{P}\left(V^{*} V\right)=1$. We can define a new state $\mathbb{Q}$ on $\mathscr{C}^{\prime}$ by $\mathbb{Q}(X)=\mathbb{P}\left(V^{*} X V\right)$, and

$$
\mathbb{Q}(X \mid \mathscr{C})=\frac{\mathbb{P}\left(V^{*} X V \mid \mathscr{C}\right)}{\mathbb{P}\left(V^{*} V \mid \mathscr{C}\right)} \quad \forall X \in \mathscr{C}^{\prime}
$$

Proof. Let $K$ be an element of $\mathscr{C}$. For any $X \in \mathscr{C}^{\prime}$ we can write

$$
\begin{aligned}
& \mathbb{P}\left(\mathbb{P}\left(V^{*} X V \mid \mathscr{C}\right) K\right)=\mathbb{P}\left(V^{*} X K V\right)=\mathbb{Q}(X K)=\mathbb{Q}(\mathbb{Q}(X \mid \mathscr{C}) K) \\
& \quad=\mathbb{P}\left(V^{*} V \mathbb{Q}(X \mid \mathscr{C}) K\right)=\mathbb{P}\left(\mathbb{P}\left(V^{*} V \mathbb{Q}(X \mid \mathscr{C}) K \mid \mathscr{C}\right)\right)=\mathbb{P}\left(\mathbb{P}\left(V^{*} V \mid \mathscr{C}\right) \mathbb{Q}(X \mid \mathscr{C}) K\right),
\end{aligned}
$$

and the result follows directly.

\footnotetext{
${ }^{1}$ That is not to say that this is the only definition used in the quantum probability literature; in fact, a more general definition, of which our definition is a special case, is very common [79]. Such a definition is very different in spirit, however, and is not motivated by the probabilistic/statistical point of view needed for filtering and feedback control.
} 
3.3. Statement of the Filtering Problem. Let us return to the two-level system coupled to the discretized electromagnetic field, as described in section 2.5. Recall that we observe the commutative process $\Delta Y(l)$, with either $Y=Y^{\Lambda}$ or $Y^{X}$ depending on what detector we choose to use, and that this process generates the commutative filtration $\mathscr{Y}$. Suppose we have been observing $Y$ up to and including time $l$. What can we infer about the atom?

The nondemolition condition allows us to make sense of this question. In the language of this section, nondemolition simply means that $j_{l}(X) \in \mathscr{Y}_{l}^{\prime}$ for any $l=$ $0, \ldots, k, X \in \mathscr{M}$. Hence the conditional expectations

$$
\pi_{l}(X)=\mathbb{P}\left(j_{l}(X) \mid \mathscr{Y}_{l}\right), \quad 0 \leq l \leq k, X \in \mathscr{M},
$$

are well defined, and we could, e.g., interpret $\pi_{l}(X)$ as the least mean-square estimate of an atomic observable $X$ at time step $l$ given the observation history.

Though it is evident that the conditional expectations $\pi_{l}(X)$ are well defined, it is quite another matter to calculate them explicitly. In principle we have a recipe which we could follow to perform this calculation: find a basis in which $j_{l}(X)$ and $\Delta Y(i), i=1, \ldots, l$, are simultaneously diagonalized, apply an expression similar to (3.1), and then reexpress the result in the original basis. Such a procedure would be extremely unpleasant, to say the least; clearly we are in need of a better way of doing this. Finding a useful explicit representation of the conditional expectations $\pi_{l}(\cdot)$ is known as the filtering problem.

Sections 5 and 6 are dedicated to the solution of the filtering problem, which we will do in two different ways (and obtain two different expressions!). Both methods give rise to recursive equations; that is, we will find filtering equations of the form $\pi_{l}(\cdot)=f\left(\pi_{l-1}(\cdot), \Delta Y(l)\right)$. This is very convenient computationally as we don't need to remember the history of observations: to calculate the current estimates $\pi_{l}(\cdot)$, all we need to know are the corresponding estimates in the previous time step $\pi_{l-1}(\cdot)$ and our observation in the current time step $\Delta Y(l)$. In other words, we can update our estimates in real time using only the current observation in each time step.

3.4. A Brief Look Ahead. The filtering problem is very interesting in itself; its solution provides us with an efficient way of estimating atomic observables from the observation data. We have an ulterior motive, however, for putting such emphasis on this problem-filtering is a fundamental part of stochastic control with partial observations, and hence plays a central role in quantum feedback control. This is the subject of sections 7-9, and we postpone a detailed discussion until then. Let us briefly sketch, however, why we might expect filtering to enter the picture.

In order to design a controller, we first have to specify the goal we are trying to achieve. Control goals are generally expressed in terms of expectations over atomic observables. For example, we might want to maximize the expected $x$-dipole moment $\mathbb{P}\left(j_{k}\left(\mu_{x}\right)\right)$ at the terminal time $t(k)=T$, or we might try to steer the dipole moment to a specific value $\alpha$ (e.g., by minimizing $\left.\mathbb{P}\left(j_{k}\left(\left(\mu_{x}-\alpha\right)^{2}\right)\right)\right)$. If we are not sure that we are going to finish running the control through time $T$, perhaps we would prefer to maximize the time-average expected dipole moment $\frac{1}{k} \sum_{l=1}^{k} \mathbb{P}\left(j_{l}\left(\mu_{x}\right)\right)$, etc. The problem is that our control can depend on the entire observation history; the optimal control can have quite a complicated dependence on the observation history!

The key observation we need is that by an elementary property of the conditional expectation, $\mathbb{P}\left(j_{l}(X)\right)=\mathbb{P}\left(\pi_{l}(X)\right)$. Rather than expressing our control goal directly in terms of the quantum model, we are free to express the same goal in terms of the filter only, as though it were the filter we wanted to control rather than the atom 
itself. It will turn out that optimal controls have a very simple dependence on the filter: the control in time step $l$ can be expressed as some (deterministic) function of the filter at time $l-1$. The filter $\pi_{l}(\cdot)$ is called an information state for the control problem, as $\pi_{l}(\cdot)$ contains all the information we need to compute the feedback law. This is discussed in detail in section 8.2.

The fact that optimal controls separate into a filtering step and a simple feedback step is known as the separation principle. The complicated dependence of the control on the observation history is completely absorbed by the filter (which is by construction only a function of the observations). By solving the filtering problem in a recursive manner, we obtain an efficient way of calculating the optimal controls. This amply motivates, beside its intrinsic interest, our interest in the filtering problem.

4. Discrete Quantum Stochastic Calculus. In the previous sections we have provided an introduction to quantum probability, introduced a toy model for the interaction of an atom with the electromagnetic field, and introduced conditional expectations and the filtering problem in the quantum setting. At this point we change gears and concentrate on developing machinery that will help us to actually solve the filtering problem (in two different ways) and ultimately treat control problems associated with our model.

We begin in this section by rewriting the repeated interactions of section 2.5 as difference equations, and we develop a "stochastic calculus" to manipulate such equations. In the discrete setting this calculus is not a very deep result: it is merely a matrix multiplication table, as can be seen from the proof below. In continuous time, however, difference equations become (quantum stochastic) differential equations and the stochastic calculus is an indispensable tool. While in continuous time linear algebra is replaced by the much more difficult functional analysis, the quantum stochastic calculus nonetheless enables one to deal with the corresponding objects using simple algebraic manipulations. Bearing in mind our goal to mirror as closely as possible the continuous theory, we will consistently use the discrete calculus in what follows. (For a similar point of view and further connections to the continuous setting, see [5, 72].)

4.I. The Discrete Quantum Itô Calculus. Let us fix once and for all the quantum probability space $\left(\mathscr{M} \otimes \mathscr{W}_{k}, \mathbb{P}\right)$ with $\mathbb{P}=\rho \otimes \phi^{\otimes k}$, which models an atom together with the electromagnetic field as in section 2.5. The algebra $\mathscr{M} \otimes \mathscr{W}_{k}$ has a natural noncommutative filtration $\left\{\mathscr{B}_{l}\right\}_{l=1, \ldots, k}$ defined by

$$
\mathscr{B}_{0}=\mathscr{M} \otimes I^{\otimes k} \subset \mathscr{B}_{1}=\mathscr{M} \otimes \mathscr{M} \otimes I^{\otimes(k-1)} \subset \cdots \subset \mathscr{B}_{k}=\mathscr{M} \otimes \mathscr{W}_{k} .
$$

The noncommutative algebra $\mathscr{B}_{l}$ contains all observables that are a function of the atom and the time slices of the field up to and including slice $l$.

A quantum process is a map from $\{0,1, \ldots, k\}$ to $\mathscr{M} \otimes \mathscr{W}_{k}$. A quantum process $L$ is called adapted if $L(i) \in \mathscr{B}_{i}$ for every $0 \leq i \leq k$. It is called predictable if $L(i) \in \mathscr{B}_{i-1}$ for every $1 \leq i \leq k$. For a quantum process $L$ we define $\Delta L$ as $\Delta L(i)=L(i)-L(i-1)$ for $1 \leq i \leq k$. These definitions are similar to those used in classical probability [83].

DEFINITION 4.1 (discrete quantum stochastic integral). Let $L$ be a predictable quantum process and let $M$ be one of the processes $A, A^{*}, \Lambda$, or $t$. The transform of $M$ by $L$, denoted $L \cdot M$, is the adapted quantum process defined by

$$
L \cdot M(l)=\sum_{i=1}^{l} L(i) \Delta M(i), \quad 1 \leq l \leq k, \quad L \cdot M(0)=0 .
$$

$L \cdot M$ is also called the discrete quantum stochastic integral of $L$ with respect to $M$. 
Note that for any discrete quantum stochastic integral as above, we can equivalently write $X=L \cdot M \Longleftrightarrow \Delta X=L \Delta M$. Note also that $L(i)$ and $\Delta M(i)$ commute by construction, so that we can always exchange the order of the (predictable) integrands and the increments $\Delta \Lambda, \Delta A, \Delta A^{*}$, and $\Delta t$. We can now state the main theorem of our "stochastic calculus."

THEOREM 4.2 (discrete quantum Itô rule). Let $L_{1}$ and $L_{2}$ be predictable quantum processes, $M_{1}, M_{2} \in\left\{A, A^{*}, \Lambda, t\right\}$, and let $X$ and $Y$ be the transforms $L_{1} \cdot M_{1}$ and $L_{2} \cdot M_{2}$, respectively. Then

$$
\Delta(X Y)(l)=X(l-1) \Delta Y(l)+\Delta X(l) Y(l-1)+\Delta X(l) \Delta Y(l),
$$

where $\Delta X(l) \Delta Y(l)$ should be evaluated according to the following discrete quantum Itô table:

\begin{tabular}{l|llll}
$\Delta X \backslash \Delta Y$ & $\Delta A$ & $\Delta \Lambda$ & $\Delta A^{*}$ & $\Delta t$ \\
\hline$\Delta A$ & 0 & $\Delta A$ & $\Delta t-\lambda^{2} \Delta \Lambda$ & $\lambda^{2} \Delta A$ \\
$\Delta \Lambda$ & 0 & $\Delta \Lambda$ & $\Delta A^{*}$ & $\lambda^{2} \Delta \Lambda$ \\
$\Delta A^{*}$ & $\lambda^{2} \Delta \Lambda$ & 0 & 0 & $\lambda^{2} \Delta A^{*}$ \\
$\Delta t$ & $\lambda^{2} \Delta A$ & $\lambda^{2} \Delta \Lambda$ & $\lambda^{2} \Delta A^{*}$ & $\lambda^{2} \Delta t$
\end{tabular}

For example, if $M_{1}=A$ and $M_{2}=A^{*}$, then $\Delta X \Delta Y=L_{1} L_{2} \Delta t-\lambda^{2} L_{1} L_{2} \Delta \Lambda$.

Proof. The quantum Itô rule follows from writing out the definition of $\Delta(X Y)$ :

$$
\begin{aligned}
\Delta(X Y)(l) & =X(l) Y(l)-X(l-1) Y(l-1) \\
& =(X(l-1)+\Delta X(l))(Y(l-1)+\Delta Y(l))-X(l-1) Y(l-1) \\
& =X(l-1) \Delta Y(l)+\Delta X(l) Y(l-1)+\Delta X(l) \Delta Y(l) .
\end{aligned}
$$

The Itô table follows directly from the definition of the increments $\Delta \Lambda, \Delta A, \Delta A^{*}, \Delta t$ in section 2.4 by explicit matrix multiplication.

The following lemma shows that transforms of $A, A^{*}$, and $\Lambda$ have zero expectation. It is a fancy way of saying that $\phi\left(\sigma_{-}\right)=\phi\left(\sigma_{+}\right)=\phi\left(\sigma_{+} \sigma_{-}\right)=0$ and $\phi(I)=1$.

Lemma 4.3. For all predictable processes $L$ we have $\mathbb{P}(L(l) \Delta t(l))=\mathbb{P}(L(l)) \lambda^{2}$ and $\mathbb{P}(L(l) \Delta A(l))=\mathbb{P}\left(L(l) \Delta A^{*}(l)\right)=\mathbb{P}(L(l) \Delta \Lambda(l))=0$ for any $1 \leq l \leq k$.

Proof. Note that $\phi\left(\sigma_{-}\right)=\phi\left(\sigma_{+}\right)=\phi\left(\sigma_{+} \sigma_{-}\right)=0$ and $\phi(I)=1$. Since $L$ is predictable, $L(i)$ and $\Delta A(i)$ are independent under $\mathbb{P}$ and we have, for $0 \leq i \leq k$,

$$
\mathbb{P}(L(i) \Delta A(i))=\lambda \mathbb{P}(L(i)) \phi\left(\sigma_{-}\right)=0 .
$$

Similar reasoning holds for $A^{*}, \Lambda$, and $t$.

4.2. Quantum Stochastic Difference Equations. We are now going to rewrite the repeated interactions of section 2.5 as difference equations. Clearly we have

$$
\Delta U(l)=U(l)-U(l-1)=\left(M_{l}-I\right) U(l-1), \quad U(0)=I .
$$

We will need to convert this expression into a more convenient form, however, in order for it to be of use in calculations.

Recall that $M_{l}$, and hence also $M_{l}-I$, is a function only of the initial system and of the $l$ th time slice of the field; indeed, this is easily seen from (2.7). But any operator $X \in \mathscr{M} \otimes \mathscr{M}$ can be written in a unique way as

$$
X=X^{ \pm} \otimes \sigma_{+} \sigma_{-}+X^{+} \otimes \sigma_{+}+X^{-} \otimes \sigma_{-}+X^{\circ} \otimes I, \quad X^{\circ,+,-, \pm} \in \mathscr{M},
$$


as $\sigma_{+} \sigma_{-}, \sigma_{+}, \sigma_{-}, I$ is a linearly independent basis for $\mathscr{M}$. By the same reasoning we find that we can write, without loss of generality,

$$
M_{l}-I=M^{ \pm} \Delta \Lambda(l)+M^{+} \Delta A^{*}(l)+M^{-} \Delta A(l)+M^{\circ} \Delta t(l),
$$

so that $M^{\circ,+,-, \pm} \in \mathscr{B}_{0}$ are uniquely determined by $L_{1,2,3}$ and $\lambda$. The functional dependence of $M^{\circ,+,-, \pm}$ on $L_{1,2,3}$ and $\lambda$ is complicated and we will not attempt to give a general expression; in the examples which we consider later it will be straightforward to calculate these matrices explicitly.

Remark 4.4. It should be emphasized that whereas $L_{1,2,3} \in \mathscr{B}_{0}$ do not depend on the time step size $\lambda, M^{\circ,+,-, \pm}$ are in fact functions of $\lambda$. As usual, we have chosen the scaling of the various parameters with $\lambda$ so that the limit as $\lambda \rightarrow 0$ gives meaningful results; in this case, it can be shown that the matrix elements of $M^{\circ,+,-, \pm}$ converge to finite values as $\lambda \rightarrow 0$, and the limiting matrices can be expressed explicitly in terms of $L_{1,2,3}$ (see [41] and other references in section 10).

We now obtain the following quantum stochastic difference equation:

$$
\Delta U(l)=\left\{M^{ \pm} \Delta \Lambda(l)+M^{+} \Delta A^{*}(l)+M^{-} \Delta A(l)+M^{\circ} \Delta t(l)\right\} U(l-1),
$$

with $U(0)=I$. It is this form of the equation that will be the most useful to us, as we can apply stochastic calculus techniques to manipulate it. We could equivalently express it in terms of discrete quantum stochastic integrals:

$$
U(l)=I+\left(M^{ \pm} U_{-}\right) \cdot \Lambda(l)+\left(M^{+} U_{-}\right) \cdot A^{*}(l)+\left(M^{-} U_{-}\right) \cdot A(l)+\left(M^{\circ} U_{-}\right) \cdot t(l),
$$

where $U_{-}(l)=U(l-1)$. This is the way that (quantum) stochastic differential equations are defined in continuous time.

To get some familiarity with calculations using the quantum Itô rule and Lemma 4.3 , let us calculate the time evolution of the expectation $\mathbb{P}\left(j_{l}(X)\right)$ for an atomic observable $X \in \mathscr{B}_{0}$. By linearity of the state we clearly have $\Delta \mathbb{P}\left(j_{l}(X)\right)=\mathbb{P}\left(\Delta j_{l}(X)\right)$, so we are going to calculate $\Delta j_{l}(X)=\Delta\left(U(l)^{*} X U(l)\right)$ using the quantum Itô rule and then calculate the expectation of the resulting expression. First, note that

$$
\Delta U(l)^{*}=U(l-1)^{*}\left\{M^{ \pm *} \Delta \Lambda(l)+M^{+*} \Delta A(l)+M^{-*} \Delta A^{*}(l)+M^{\circ *} \Delta t(l)\right\} .
$$

Then we calculate, using the quantum Itô rule,

$$
\begin{aligned}
& \Delta\left(U(l)^{*} X U(l)\right)=(\cdots) \Delta \Lambda(l)+(\cdots) \Delta A^{*}(l)+(\cdots) \Delta A(l) \\
& \quad+U(l-1)^{*}\left\{M^{+*} X M^{+}+\lambda^{2} M^{\circ *} X M^{\circ}+M^{\circ *} X+X M^{\circ}\right\} U(l-1) \Delta t(l) .
\end{aligned}
$$

We didn't bother to calculate the $\Delta \Lambda, \Delta A, \Delta A^{*}$ terms; by Lemma 4.3 these vanish anyway when we take the expectation with respect to $\mathbb{P}$, so we only need the $\Delta t$ term. Hence we obtain, for any $X \in \mathscr{B}_{0}$,

$$
\frac{\Delta \mathbb{P}\left(j_{l}(X)\right)}{\Delta t}=\mathbb{P}\left(j_{l-1}(\mathcal{L}(X))\right)
$$

where the discrete Lindblad generator $\mathcal{L}(\cdot)$ is defined by

$$
\mathcal{L}(X)=M^{+*} X M^{+}+\lambda^{2} M^{\circ *} X M^{\circ}+M^{\circ *} X+X M^{\circ} .
$$

Copyright (C) by SIAM. Unauthorized reproduction of this article is prohibited. 
Remark 4.5. Let $X_{t}$ be a classical, continuous time Markov diffusion, and define the semigroup $P_{t}(f)=\mathbf{E} f\left(X_{t}\right)$. Then (under sufficient regularity conditions) $d P_{t}(f) / d t=P_{t}(\mathcal{L} f)$, where $\mathcal{L}$ is the infinitesimal generator of the Markov diffusion. Equation (4.3) is strongly reminiscent of this formula, and indeed the continuous time version of the formula plays an equivalent role in quantum probability. In fact, the semigroup property (expressed in "differential" form (4.3)) suggests that we should interpret our repeated interaction model as a "quantum Markov process," which can be given a precise meaning [61], but we will not do so here.

Note that we are not allowed to choose $M^{ \pm,+,-, \circ}$ arbitrarily: these matrices must be chosen in such a way that the solution $U(l)$ of the corresponding difference equation is unitary. Obtaining $M^{ \pm,+,-, \circ}$ directly from $L_{1,2,3}$, as we will do in the examples below, ensures that this is the case. One could establish general conditions on $M^{ \pm,+,-, \circ}$ to ensure that $U(l)$ is unitary, but we will not need these in what follows. There is one particular necessary condition, however, that we will often use.

Lemma 4.6. For any repeated interaction model, $\mathcal{L}(I)=0$.

Proof. As $U(l)$ is unitary, $j_{l}(I)=U(l)^{*} U(l)=I$ for any $l$. By (4.3) we have

$$
0=\frac{\mathbb{P}\left(j_{1}(I)-j_{0}(I)\right)}{\Delta t}=\mathbb{P}\left(j_{0}(\mathcal{L}(I))\right)=\rho(\mathcal{L}(I)) .
$$

But this must hold for any initial state $\rho$, so $\mathcal{L}(I)=0$ identically.

4.3. Examples. Let us now return to the examples of section 2.6. We will calculate the difference equations for the unitary evolution $U(l)$ explicitly.

Spontaneous Emission. Recall that in this case (with $2 \kappa=1$ )

$$
M_{l}=\exp \left(\sigma_{-} \Delta A^{*}(l)-\sigma_{+} \Delta A(l)\right) .
$$

As $M_{l}$ is of the form $P \otimes I^{\otimes l-1} \otimes Q \otimes I^{\otimes k-l}$, we can read off the form of $M_{l}$ by calculating the matrix exponential of

$$
\sigma_{-} \otimes \lambda \sigma_{+}-\sigma_{+} \otimes \lambda \sigma_{-}=\left(\begin{array}{cccc}
0 & 0 & 0 & 0 \\
0 & 0 & \lambda & 0 \\
0 & -\lambda & 0 & 0 \\
0 & 0 & 0 & 0
\end{array}\right) .
$$

Performing this calculation explicitly, we get

$$
\begin{aligned}
& e^{\sigma_{-} \otimes \lambda \sigma_{+}-\sigma_{+} \otimes \lambda \sigma_{-}}=\left(\begin{array}{cccc}
1 & 0 & 0 & 0 \\
0 & \cos \lambda & \sin \lambda & 0 \\
0 & -\sin \lambda & \cos \lambda & 0 \\
0 & 0 & 0 & 1
\end{array}\right) \\
& =\left(\begin{array}{cc}
1 & 0 \\
0 & \cos \lambda
\end{array}\right) \otimes \sigma_{+} \sigma_{-}+\sin \lambda \sigma_{-} \otimes \sigma_{+}-\sin \lambda \sigma_{+} \otimes \sigma_{-}+\left(\begin{array}{cc}
\cos \lambda & 0 \\
0 & 1
\end{array}\right) \otimes \sigma_{-} \sigma_{+} \\
& =(1-\cos \lambda) \sigma_{z} \otimes \sigma_{+} \sigma_{-}+\sin \lambda \sigma_{-} \otimes \sigma_{+}-\sin \lambda \sigma_{+} \otimes \sigma_{-}+\left(\begin{array}{cc}
\cos \lambda & 0 \\
0 & 1
\end{array}\right) \otimes I,
\end{aligned}
$$

where we have used $\sigma_{-} \sigma_{+}=I-\sigma_{+} \sigma_{-}$. Hence we obtain

$M_{l}=(1-\cos \lambda) \sigma_{z} \Delta \Lambda(l)+\frac{\sin \lambda}{\lambda}\left(\sigma_{-} \Delta A^{*}(l)-\sigma_{+} \Delta A(l)\right)+\frac{\cos \lambda-1}{\lambda^{2}} \sigma_{+} \sigma_{-} \Delta t(l)+I$. 
We can now immediately find the coefficients in the quantum stochastic difference equation for the spontaneous emission model:

$$
\begin{aligned}
& M^{ \pm}=(1-\cos \lambda) \sigma_{z}, \quad M^{+}=\frac{\sin \lambda}{\lambda} \sigma_{-}, \\
& M^{-}=-\frac{\sin \lambda}{\lambda} \sigma_{+}, \quad M^{\circ}=\frac{\cos \lambda-1}{\lambda^{2}} \sigma_{+} \sigma_{-} .
\end{aligned}
$$

Dispersive Interaction. Recall that in this case (with $g=1$ )

$$
M_{l}=\exp \left(\sigma_{z}\left(\Delta A^{*}(l)-\Delta A(l)\right)\right) .
$$

We proceed as in the case of spontaneous emission. Starting from

$$
\sigma_{z} \otimes \lambda\left(\sigma_{+}-\sigma_{-}\right)=\left(\begin{array}{cccc}
0 & 0 & \lambda & 0 \\
0 & 0 & 0 & -\lambda \\
-\lambda & 0 & 0 & 0 \\
0 & \lambda & 0 & 0
\end{array}\right)
$$

we calculate the matrix exponential

$$
e^{\sigma_{z} \otimes \lambda\left(\sigma_{+}-\sigma_{-}\right)}=\left(\begin{array}{cccc}
\cos \lambda & 0 & \sin \lambda & 0 \\
0 & \cos \lambda & 0 & -\sin \lambda \\
-\sin \lambda & 0 & \cos \lambda & 0 \\
0 & \sin \lambda & 0 & \cos \lambda
\end{array}\right)=\cos \lambda I+\sin \lambda \sigma_{z} \otimes\left(\sigma_{+}-\sigma_{-}\right) .
$$

Hence we obtain

$$
M_{l}=\frac{\sin \lambda}{\lambda} \sigma_{z}\left(\Delta A^{*}(l)-\Delta A(l)\right)+\frac{\cos \lambda-1}{\lambda^{2}} \Delta t(l)+I .
$$

We can now immediately see the coefficients in the quantum stochastic difference equation for the dispersive interaction model:

$$
M^{ \pm}=0, \quad M^{+}=-M^{-}=\frac{\sin \lambda}{\lambda} \sigma_{z}, \quad M^{\circ}=\frac{\cos \lambda-1}{\lambda^{2}} I .
$$

A First Simulation. To get some idea for the mean behavior of our two examples, let us calculate the expectation of the energy observable $\mathbb{P}\left(j_{l}(H)\right)$ as a function of time. The expectation of any atomic observable is given by (4.3). To simulate (4.3) directly, we can use a standard technique which will be used several times in what follows. Note that $\mathbb{P}\left(j_{l}(X)\right)$ is a linear function of $X$. Hence we can always find a $2 \times 2$ matrix $\tau_{l}$ such that $\operatorname{Tr}\left[\tau_{l} X\right]=\mathbb{P}\left(j_{l}(X)\right)$ for every $X$. Substituting this expression into (4.3), we obtain explicitly a recursive equation for $\tau_{l}$ :

$$
\frac{\Delta \tau_{l}}{\Delta t}=M^{+} \tau_{l-1} M^{+*}+\lambda^{2} M^{\circ} \tau_{l-1} M^{\circ *}+M^{\circ} \tau_{l-1}+\tau_{l-1} M^{\circ *},
$$

where $\tau_{0}$ is the density matrix corresponding to the initial state $\rho: \rho(X)=\operatorname{Tr}\left[\tau_{0} X\right]$ for every $X$. This equation is called the (discrete) master equation and plays a similar role to the forward Kolmogorov (or Fokker-Planck) equation in classical diffusion theory. The recursion is easily implemented in a computer program, and the solution allows us to calculate $\mathbb{P}\left(j_{l}(X)\right)$ for any $X$. For the spontaneous emission case, the expected energy $\mathbb{P}\left(j_{l}(H)\right)=\operatorname{Tr}\left[\tau_{l} H\right]$ is plotted in Figure 4.1. 


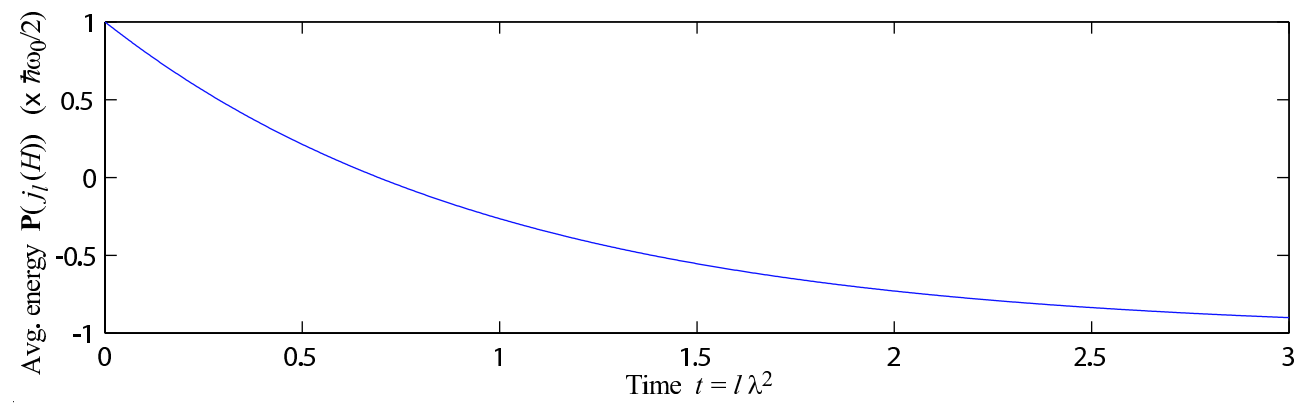

Fig. 4.I The expectation of the energy of a spontaneously-emitting atom as a function of time. The initial state $\rho$ is chosen in such a way that the atomic energy takes its maximal value $+\hbar \omega_{0} / 2$ with unit probability at $t=0$, i.e., $\rho(x)=\operatorname{Tr}\left[\sigma_{+} \sigma_{-} X\right]$. The expected energy decays exponentially until it reaches its minimum value $-\hbar \omega_{0} / 2$. The time scale used for the calculation is $\lambda^{-2}=300$.

We have used this opportunity as an excuse to introduce the master equation; in this simple case, however, we can obtain the result of Figure 4.1 much more directly. Let us calculate $\mathcal{L}(H)=\left(\hbar \omega_{0} / 2\right) \mathcal{L}\left(\sigma_{z}\right)$ in the case of spontaneous emission:

$$
\mathcal{L}\left(\sigma_{z}\right)=-\frac{2 \sin ^{2} \lambda}{\lambda^{2}} \sigma_{+} \sigma_{-}=-\frac{\sin ^{2} \lambda}{\lambda^{2}}\left(\sigma_{z}+I\right)
$$

Hence we have

$$
\mathbb{P}\left(j_{l}\left(\sigma_{z}\right)\right)+1=\Delta \mathbb{P}\left(j_{l}\left(\sigma_{z}\right)\right)+\mathbb{P}\left(j_{l-1}\left(\sigma_{z}\right)\right)+1=\cos ^{2} \lambda\left(\mathbb{P}\left(j_{l-1}\left(\sigma_{z}\right)\right)+1\right) .
$$

Recursing this relation, we find that the expected energy decays geometrically:

$$
\mathbb{P}\left(j_{l}(H)\right)=-\frac{\hbar \omega_{0}}{2}+\left(\rho(H)+\frac{\hbar \omega_{0}}{2}\right)\left(\cos ^{2} \lambda\right)^{l} .
$$

This expression coincides exactly with the plot in Figure 4.1.

In the dispersive case, the time dependence of the mean energy is trivial; it is not difficult to check that in this case $\mathcal{L}(H)=0$. This is not too surprising, as we have suppressed the atom's ability to radiate its excess energy by placing it in an optical cavity. In fact, using $M_{l}=\exp \left(\sigma_{z}\left(\Delta A^{*}(l)-\Delta A(l)\right)\right)$ we find that

$$
j_{l}(H)=M_{1}^{*} \cdots M_{l-1}^{*} M_{l}^{*} H M_{l} M_{l-1} \cdots M_{1}=H .
$$

Evidently not only the expected energy, but even the energy as a random variable, is conserved in the dispersive case.

5. The Martingale Method. In this section we will provide a solution to the filtering problem using martingale methods. The key tools we need are minor variations on two classical theorems. First, there is a theorem of Doob which states that any adapted process can be decomposed as the sum of a predictable process and a martingale; we extend this theorem slightly to a class of nondemolition processes. Applying this theorem to the conditional expectation $\pi_{l}(X)$, we can immediately identify the corresponding predictable part. Next, the martingale representation theorem tells us that any martingale can be written as a stochastic integral of some predictable process. Consequently, we can also identify the martingale part of $\pi_{l}(X)$ in terms of a 
stochastic integral. The recursive nature of the solution is the motivation behind this procedure: the predictable parts will exactly turn out to be functions of the filter in the previous time step, whereas the increment in the discrete stochastic integral will be directly related to the observation increment.

For excellent introductions to the use of martingale methods in classical filtering theory we refer to $[29,59]$; definitive treatments can be found in [64, 55]. Martingale methods for quantum filtering were introduced in [12] and used in $[19,20]$. The treatment below is a discretized version of the latter.

5.I. The Classic Theorems. Recall that $\left\{\mathscr{Y}_{l}\right\}$ is the commutative filtration generated by the observations.

Definition 5.1. A quantum process $X$ is called nondemolished (by the observations $Y$ ) if $X(l)$ is in the commutant of $\mathscr{Y}_{l}$ for $0 \leq l \leq k$. A nondemolished quantum process $H$ is called an nd-martingale (with respect to the filtration $\mathscr{Y}_{0 \leq l \leq k}$ ) if

$$
\mathbb{P}\left(H(l) \mid \mathscr{Y}_{m}\right)=\mathbb{P}\left(H(m) \mid \mathscr{Y}_{m}\right) \quad \forall 0 \leq m \leq l \leq k .
$$

$A$ quantum process $A$ is called $\mathscr{Y}$-predictable if $A(l)$ is in $\mathscr{Y}_{l-1}$ for $1 \leq l \leq k$, and $A$ is called $\mathscr{Y}$-adapted if $A(l)$ is in $\mathscr{Y}$ for $1 \leq l \leq k$. An nd-martingale that is additionally $\mathscr{Y}$-adapted is called a $\mathscr{Y}$-martingale or simply a martingale.

The nondemolition requirement ensures that the conditional expectations in the definition of an nd-martingale are well defined: $\mathscr{Y}_{m} \subset \mathscr{Y}_{l}$ implies $\mathscr{Y}_{m}^{\prime} \supset \mathscr{Y}_{l}^{\prime}$, so $H(l) \in \mathscr{Y}_{l}^{\prime}$ implies $H(l) \in \mathscr{Y}_{m}^{\prime}$. Note also that, by construction, any $\mathscr{Y}$-predictable or $\mathscr{Y}$-adapted process is a function of the observations and is hence commutative.

Remark 5.2. The concept of a $\mathscr{Y}$-martingale, being $\mathscr{Y}$-adapted and hence a classical process, coincides with the classical notion of a martingale. An nd-martingale ("nd" for nondemolition) is used in a slightly broader sense; we will encounter an example below of a commutative nd-martingale that is not a martingale. It will be convenient to use this terminology, as we will use the following theorem.

Theorem 5.3 (Doob decomposition). Let $X$ be a quantum process nondemolished by the observations $Y$. Then $X$ has the following Doob decomposition:

$$
X(l)=X(0)+A(l)+H(l), \quad l=1, \ldots, k,
$$

where $H$ is an nd-martingale (w.r.t. $\mathscr{Y}_{0 \leq l \leq k}$ ) null at 0 and $A$ is a $\mathscr{Y}$-predictable process null at 0 . Moreover, the decomposition is unique modulo indistinguishability.

The proof is very similar to its classical counterpart [83].

Proof. Suppose $X$ has a Doob decomposition as in the theorem. Then

$$
\begin{aligned}
& \mathbb{P}\left(X(l)-X(l-1) \mid \mathscr{Y}_{l-1}\right) \\
& \quad=\mathbb{P}\left(H(l)-H(l-1) \mid \mathscr{Y}_{l-1}\right)+\mathbb{P}\left(A(l)-A(l-1) \mid \mathscr{Y}_{l-1}\right)=A(l)-A(l-1),
\end{aligned}
$$

where we have used that $H$ is an nd-martingale and that $A$ is predictable. Hence

$$
A(l)=\sum_{m=1}^{l} \mathbb{P}\left(X(m)-X(m-1) \mid \mathscr{Y}_{m-1}\right), \quad A(0)=0 .
$$

For any nondemolished process $X$, define the predictable process $A(l)$ as in (5.2), and define $H(l)=X(l)-X(0)-A(l)$. Then it is easily verified that $H$ is an nd-martingale, hence we have explicitly constructed a Doob decomposition. 
To prove uniqueness, suppose that $X(l)=X(0)+\tilde{A}(l)+\tilde{H}(l)$ is another Doob decomposition. Then $A(l)-\tilde{A}(l)=H(l)-\tilde{H}(l)$ for $1 \leq l \leq k$, and hence

$$
\begin{aligned}
& A(l)-\tilde{A}(l)=\mathbb{P}\left(A(l)-\tilde{A}(l) \mid \mathscr{Y}_{l-1}\right) \\
& =\mathbb{P}\left(H(l)-\tilde{H}(l) \mid \mathscr{Y}_{l-1}\right)=\mathbb{P}\left(H(l-1)-\tilde{H}(l-1) \mid \mathscr{Y}_{l-1}\right) \\
& \quad=\mathbb{P}\left(A(l-1)-\tilde{A}(l-1) \mid \mathscr{Y}_{l-1}\right)=A(l-1)-\tilde{A}(l-1),
\end{aligned}
$$

where we have used predictability of $A, \tilde{A}$ and the nd-martingale property of $H, \tilde{H}$. But as $A(0)=\tilde{A}(0)=0$, we obtain by induction $0=A(l)-\tilde{A}(l)=H(l)-\tilde{H}(l)$ for $1 \leq l \leq k$. Hence $A=\tilde{A}$ and $H=\tilde{H}$ with probability 1 (as the conditional expectations in (5.3) are only defined up to a choice of version).

Remark 5.4. It should be noted that the Doob decomposition depends crucially on the choice of filtration $\mathscr{Y}$, which is demonstrated by the following trivial example. Consider the (commutative) filtration $\left\{\mathscr{I}_{l}\right\}$ with $\mathscr{I}_{l}=\operatorname{alg}\{I\}$ for any $l$. As $\mathscr{I}_{l}$ contains only multiples of the identity, the commutant is the entire algebra $\mathscr{I}^{\prime}=\mathscr{M} \otimes \mathscr{W}_{k}$. Hence any process $X$ is nondemolished by $\mathscr{I}$, and as $\mathbb{P}\left(X(l) \mid \mathscr{I}_{m}\right)=\mathbb{P}(X(l))$ for any $l, m$, any process with constant expectation $\mathbb{P}(X(l))=\mathbb{P}(X(m))$ is an nd-martingale with respect to $\left\{\mathscr{I}_{l}\right\}$ (but not necessarily an $\mathscr{I}$-martingale!). Using $\mathscr{I}$ as the filtration, we obtain the Doob decomposition $A(l)=\mathbb{P}(X(l)-X(0))$ and $H(l)=X(l)-X(0)-$ $\mathbb{P}(X(l)-X(0))$ for any process $X$. Clearly this decomposition is different than the Doob decomposition with respect to $\mathscr{Y}$, but note that $H(l)$ is not an nd-martingale with respect to $\left\{\mathscr{Y}_{l}\right\}$, so uniqueness is not violated. The moral of the story is that we have to be careful to specify the filtration with respect to which we decompose a process. In the following, this will always be the filtration $\left\{\mathscr{Y}_{l}\right\}$ generated by the observations.

Let $X \in \mathscr{B}_{0}$. Applying the Doob decomposition to $\pi_{l}(X)$ and $Y(l)$ gives

$$
\begin{array}{ccl}
\pi_{l}(X)=\rho(X)+B(l)+H(l), & \text { i.e., } & \Delta \pi_{l}(X)=\Delta B(l)+\Delta H(l), \\
Y(l)=Y(0)+C(l)+\tilde{Y}(l), & \text { i.e., } & \Delta Y(l)=\Delta C(l)+\Delta \tilde{Y}(l),
\end{array}
$$

where $B$ and $C$ are predictable processes null at 0 and $\tilde{Y}$ and $H$ are $\mathscr{Y}$-martingales null at 0 . The process $\tilde{Y}$ is called the innovating martingale. In the next two subsections we will investigate the processes $C$ and $\tilde{Y}$ in more detail for both the counting and homodyne detection cases.

Lemma 5.5. The predictable process $B$ in the decomposition of $\pi_{l}(X)$ is given by

$$
\Delta B(l)=\pi_{l-1}(\mathcal{L}(X)) \Delta t(l), \quad 1 \leq l \leq k,
$$

where $\mathcal{L}$ is the discrete Lindblad generator of (4.4).

Proof. By (5.1), we have $\Delta B(l)=\mathbb{P}\left(\Delta \pi_{l}(X) \mid \mathscr{Y}_{l-1}\right)=\mathbb{P}\left(\Delta j_{l}(X) \mid \mathscr{Y}_{l-1}\right)$. To calculate the latter, let $K$ be an element in $\mathscr{Y}_{l-1}$. Using (4.2), we obtain

$$
\mathbb{P}\left(K \Delta j_{l}(X)\right)=\mathbb{P}\left(K j_{l-1}(\mathcal{L}(X)) \Delta t(l)\right)=\mathbb{P}\left(K \pi_{l-1}(\mathcal{L}(X)) \Delta t(l)\right) .
$$

As this holds for any $K \in \mathscr{Y}_{l-1}$, and as $\pi_{l-1}(\mathcal{L}(X)) \in \mathscr{Y}_{l-1}$, the statement of the lemma follows from the definition of the conditional expectation.

At this point, we have the expression

$$
\Delta \pi_{l}(X)=\pi_{l-1}(\mathcal{L}(X)) \Delta t(l)+\Delta H(l) .
$$

Copyright (c) by SIAM. Unauthorized reproduction of this article is prohibited. 
This is almost a recursive equation; what we would like to do is write something like $\Delta H(l)=f\left(\pi_{l-1}(\cdot)\right) \Delta Y(l)$, as in that case we could use this equation to calculate $\pi_{l}(\cdot)$ using only $\pi_{l-1}(\cdot)$ and $\Delta Y(l)$. The problem is that $f\left(\pi_{l-1}(\cdot)\right) \Delta Y(l)$ does not define a martingale, but $f\left(\pi_{l-1}(\cdot)\right) \Delta \tilde{Y}(l)$ does! This suggests that we should try to represent $H$ as a discrete stochastic integral with respect to the innovating martingale $\tilde{Y}(l)$. The martingale representation theorem shows that this is always possible.

THEOREM 5.6 (martingale representation). Let $\tilde{Y}$ be the innovating martingale and let $H$ be a $\mathscr{Y}$-martingale null at 0 . Then there exists a $\mathscr{Y}$-predictable process $\Xi$ such that $\Delta H(l)=\Xi(l) \Delta \tilde{Y}(l), l=1, \ldots, k$, modulo indistinguishability.

The following proof is reminiscent of [83, pp. 154-155], but the details of the argument are a little more delicate in our case.

Proof. As all the observables in the theorem are contained in the full observation algebra $\mathscr{Y}_{k}$, which is commutative, this is essentially a classical problem. It will be convenient for the proof to treat it as such, i.e., applying the spectral theorem to $\left(\mathscr{Y}_{k}, \mathbb{P}\right)$ gives the classical probability space $(\Omega, \mathcal{F}, \mathbf{P})$, the filtration $\left\{\mathscr{Y}_{l}\right\}$ gives rise to the classical filtration $\left\{\mathcal{Y}_{l}\right\}$, and we will write $y_{l}=\iota(Y(l)), \tilde{y}_{l}=\iota(\tilde{Y}(l))$, and $h_{l}=\iota(H(l))$. It will be convenient to write $\omega_{l}=\iota(\Delta Y(l))=y_{l}-y_{l-1}$.

We will make fundamental use of the following fact: $\omega_{l}$ takes one of two values $\left\{\omega^{+}, \omega^{-}\right\}$for every $l=1, \ldots, k$. To see this, recall that $\Delta Y(l)=U(l)^{*} \Delta Z(l) U(l)$, where $\Delta Z(l)$ is one of $\Delta A(l)+\Delta A^{*}(l)$ or $\Delta \Lambda(l)$ (in fact, any observable $\Delta Z(l)$ of the form $(Z)_{l} \in \mathscr{W}_{k}, Z \in \mathscr{M}$, would do, provided $Z$ is not a multiple of the identity). Hence $\Delta Z(l)$ has two distinct eigenvalues, and as unitary rotation leaves the spectrum of an operator invariant, so does $\Delta Y(l)$. It follows that $\iota(\Delta Y(l))$ is a two-state random variable. We will write $p_{l}^{ \pm}=\mathbf{P}\left(\omega_{l}=\omega^{ \pm} \mid \mathcal{Y}_{l-1}\right)$ for the conditional probability that $\omega_{l}=\omega^{ \pm}$given that we have observed $\omega_{1}, \ldots, \omega_{l-1}$.

Now recall that $\mathscr{Y}_{l}$ is the algebra generated by $\Delta Y(i), i=1, \ldots, l$. Hence every $\mathcal{Y}_{l}$-measurable random variable can be written as a function of $\omega_{i}, i=1, \ldots, l$. In particular, $\tilde{y}_{l}=\tilde{y}_{l}\left(\omega_{1}, \ldots, \omega_{l}\right)$ and $h_{l}=h_{l}\left(\omega_{1}, \ldots, \omega_{l}\right)$. We would like to find a $\xi_{l}\left(\omega_{1}, \ldots, \omega_{l-1}\right)$ (independent of $\omega_{l}$, hence predictable) that satisfies

$$
\Delta h_{l}\left(\omega_{1}, \ldots, \omega_{l}\right)=\xi_{l}\left(\omega_{1}, \ldots, \omega_{l-1}\right) \Delta \tilde{y}_{l}\left(\omega_{1}, \ldots, \omega_{l}\right)
$$

To proceed, we split $\Omega$ into three disjoint subsets $\Omega_{1,2,3}$ and define the random variable $\xi_{l}$ separately on each set.

Case 1: $\Omega_{1}=\left\{\omega \in \Omega: \Delta \tilde{y}_{l}\left(\omega_{1}, \ldots, \omega_{l-1}, \omega^{ \pm}\right) \neq 0\right\}$. Let us suppose that $\xi_{l}$ exists. Then evidently on $\Omega_{1}$,

$$
\xi_{l}\left(\omega_{1}, \ldots, \omega_{l-1}\right)=\frac{\Delta h_{l}\left(\omega_{1}, \ldots, \omega_{l}\right)}{\Delta \tilde{y}_{l}\left(\omega_{1}, \ldots, \omega_{l}\right)}
$$

Existence of $\xi_{l}$ is thus verified by construction if we can show that the right-hand side is independent of $\omega_{l}$. To this end, we express the martingale property of $h_{l}$ as

$\Delta h_{l}\left(\omega_{1}, \ldots, \omega_{l-1}, \omega^{+}\right) p_{l}^{+}\left(\omega_{1}, \ldots, \omega_{l-1}\right)+\Delta h_{l}\left(\omega_{1}, \ldots, \omega_{l-1}, \omega^{-}\right) p_{l}^{-}\left(\omega_{1}, \ldots, \omega_{l-1}\right)=0$

a.s., where the left-hand side is simply the expression for $\mathbf{E}_{\mathbf{P}}\left(\Delta h_{l} \mid \mathcal{Y}_{l-1}\right)$. Similarly,

$$
\Delta \tilde{y}_{l}\left(\omega_{1}, \ldots, \omega_{l-1}, \omega^{+}\right) p_{l}^{+}\left(\omega_{1}, \ldots, \omega_{l-1}\right)+\Delta \tilde{y}_{l}\left(\omega_{1}, \ldots, \omega_{l-1}, \omega^{-}\right) p_{l}^{-}\left(\omega_{1}, \ldots, \omega_{l-1}\right)=0
$$


a.s., as $\tilde{y}_{l}$ is a martingale. Note that necessarily $p_{l}^{ \pm} \neq 0$ a.s. on $\Omega_{1}$. Hence we obtain

$$
\begin{aligned}
\Delta h_{l}\left(\omega_{1}, \ldots, \omega_{l-1}, \omega^{+}\right) & =-\Delta h_{l}\left(\omega_{1}, \ldots, \omega_{l-1}, \omega^{-}\right) \frac{p_{l}^{-}\left(\omega_{1}, \ldots, \omega_{l-1}\right)}{p_{l}^{+}\left(\omega_{1}, \ldots, \omega_{l-1}\right)}, \\
\Delta \tilde{y}_{l}\left(\omega_{1}, \ldots, \omega_{l-1}, \omega^{+}\right) & =-\Delta \tilde{y}_{l}\left(\omega_{1}, \ldots, \omega_{l-1}, \omega^{-}\right) \frac{p_{l}^{-}\left(\omega_{1}, \ldots, \omega_{l-1}\right)}{p_{l}^{+}\left(\omega_{1}, \ldots, \omega_{l-1}\right)} .
\end{aligned}
$$

Dividing the first by the second expression, the independence of $\xi_{l}$ from $\omega_{l}$ follows.

Case 2: $\Omega_{2}=\left\{\omega \in \Omega: p_{l}^{+} \in\{0,1\}\right\}$. Using the martingale property of $h_{l}$ and $\tilde{y}_{l}$ as above, we conclude that on $\Omega_{2}$ we have $\Delta h_{l}=\Delta \tilde{y}_{l}=0$ a.s. Hence (5.5) holds regardless of the value we assign to $\xi_{l}$.

Case 3: $\Omega_{3}=\Omega \backslash\left(\Omega_{1} \cup \Omega_{2}\right)$. We will show that $\mathbf{P}\left(\Omega_{3}\right)=0$, so that we do not need to worry about defining $\xi_{l}$ on this set. Since on $\Omega_{3}$ we have $p_{l}^{ \pm} \neq 0$ but one of $\Delta \tilde{y}_{l}\left(\omega_{1}, \ldots, \omega_{l-1}, \omega^{ \pm}\right)=0$, using the martingale property as above allows us to conclude that $\Delta \tilde{y}_{l}=0$ a.s. on $\Omega_{3}$. Recall that $\omega_{l}=\Delta y_{l}=\Delta c_{l}+\Delta \tilde{y}_{l}$, where $\Delta c_{l}$ is predictable. Then $\omega_{l}=\Delta c_{l}\left(\omega_{1}, \ldots, \omega_{l-1}\right)$ a.s. on $\Omega_{3}$. But this would imply that $\omega_{l}=\mathbf{E}_{\mathbf{P}}\left(\omega_{l} \mid \mathcal{Y}_{l-1}\right)=\omega^{+} p_{l}^{+}+\omega^{-} p_{l}^{-}$, and as $p_{l}^{ \pm} \neq 0$ we could conclude that $\omega_{l} \neq \omega^{ \pm}$. Hence we have a contradiction.

We have now shown how to define $\xi_{l}$ that satisfies (5.5) except possibly on a set of measure zero. Setting $\Xi(l)=\iota^{-1}\left(\xi_{l}\right)$, the theorem is proved.

Though the proof of the discrete martingale representation theorem is in principle constructive, it is not advisable to follow this complicated procedure in order to calculate the predictable process $\Xi$. Instead we will calculate $\Xi$ using a standard trick of filtering theory, and it will turn out to depend only on the conditional expectations in the previous time step. Putting everything together, we obtain a recursive relation with which we can update our conditional expectations of atomic operators given the conditional expectations at the previous time step and the observation result at the present time step. This recursion is called the discrete quantum filtering equation. As the predictable processes $C$ and $\Xi$ depend on the nature of the observations, we consider separately the homodyne and photon-counting cases.

5.2. Homodyne Detection. Let us first consider a homodyne detection setup, i.e., an experimental setup that allows us to observe

$$
Y^{X}(l)=U(l)^{*}\left(A(l)+A^{*}(l)\right) U(l), \quad 0 \leq l \leq k .
$$

We begin by finding the predictable process $C$ in the Doob decomposition of $Y^{X}$.

Lemma 5.7. The predictable process $C$ in the decomposition of $Y^{X}$ is given by

$$
\Delta C(l)=\pi_{l-1}\left(M^{+}+M^{+*}+\lambda^{2} M^{\circ *} M^{+}+\lambda^{2} M^{+*} M^{\circ}\right) \Delta t(l), \quad 1 \leq l \leq k .
$$

Proof. By (5.1), we have $\Delta C(l)=\mathbb{P}\left(\Delta Y^{X}(l) \mid \mathscr{Y}_{l-1}\right)$. To calculate the latter, let $K$ be an element in $\mathscr{Y}_{l-1}$; we would like to find an expression for $\mathbb{P}\left(K \Delta Y^{X}(l)\right)$. To this end, we calculate using the discrete quantum Itô rules that

$$
\begin{aligned}
\Delta\left(\left(A(l)+A^{*}(l)\right) U(l)\right) & =(\cdots) \Delta \Lambda(l)+(\cdots) \Delta A(l) \\
+\{I+(A(l-1) & \left.\left.+A^{*}(l-1)\right) M^{+}+\lambda^{2} M^{\circ}\right\} U(l-1) \Delta A^{*}(l) \\
& +\left\{\left(A(l-1)+A^{*}(l-1)\right) M^{\circ}+M^{+}\right\} U(l-1) \Delta t(l)
\end{aligned}
$$

Copyright $@$ ㅇ by SIAM. Unauthorized reproduction of this article is prohibited. 
where we have retained the relevant terms. Consequently, we calculate

$$
\begin{array}{rl}
\Delta\left(U(l)^{*}\left(A(l)+A^{*}(l)\right) U(l)\right) & =(\cdots) \Delta \Lambda(l)+(\cdots) \Delta A^{*}(l)+(\cdots) \Delta A(l) \\
+U(l-1)^{*} & \mathcal{L}(I)\left(A(l-1)+A^{*}(l-1)\right) U(l-1) \Delta t(l) \\
& +j_{l-1}\left(M^{+}+M^{+*}+\lambda^{2} M^{\circ *} M^{+}+\lambda^{2} M^{+*} M^{\circ}\right) \Delta t(l),
\end{array}
$$

where $\mathcal{L}(X)$ is the discrete Lindblad generator. But $\mathcal{L}(I)$ vanishes by Lemma 4.6. Hence we obtain

$$
\mathbb{P}\left(K \Delta Y^{X}(l)\right)=\mathbb{P}\left(K j_{l-1}\left(M^{+}+M^{+*}+\lambda^{2} M^{\circ *} M^{+}+\lambda^{2} M^{+*} M^{\circ}\right) \Delta t(l)\right)
$$

using Lemma 4.3, or equivalently (using $K \in \mathscr{Y}_{l-1}$ )

$$
\mathbb{P}\left(K \Delta Y^{X}(l)\right)=\mathbb{P}\left(K \pi_{l-1}\left(M^{+}+M^{+*}+\lambda^{2} M^{\circ *} M^{+}+\lambda^{2} M^{+*} M^{\circ}\right) \Delta t(l)\right) .
$$

As this holds for any $K \in \mathscr{Y}_{l-1}$, and as $\pi_{l-1}(\cdots) \in \mathscr{Y}_{l-1}$, the statement of the lemma follows from the definition of the conditional expectation.

From Theorem 5.6 we know that $\Delta H=\Xi \Delta \tilde{Y}$ for some predictable process $\Xi$. It remains to determine $\Xi$; we approach this problem using a standard technique. Since $\mathbb{P}\left(j_{l}(X) Y(l) \mid \mathscr{Y}_{l}\right)=\pi_{l}(X) Y(l)$, the uniqueness of the Doob decomposition ensures that $j_{l}(X) Y(l)$ and $\pi_{l}(X) Y(l)$ have equal predictable parts. We write $D$ and $E$ for the predictable processes in the Doob decomposition of $j_{l}(X) Y(l)$ and $\pi_{l}(X) Y(l)$, respectively. Solving the equation $\Delta D=\Delta E$ will then allow us to determine $\Xi$.

Lemma 5.8. For any $X \in \mathscr{B}_{0}$, define

$$
\mathcal{J}(X)=X M^{+}+M^{+*} X+\lambda^{2} M^{\circ *} X M^{+}+\lambda^{2} M^{+*} X M^{\circ},
$$

so that $\Delta C(l)=\pi_{l-1}(\mathcal{J}(I)) \Delta t(l)$. Then we can write

$$
\Xi(l)=\frac{\pi_{l-1}(\mathcal{J}(X))-\pi_{l-1}\left(X+\lambda^{2} \mathcal{L}(X)\right) \pi_{l-1}(\mathcal{J}(I))}{I-\lambda^{2} \pi_{l-1}(\mathcal{J}(I))^{2}} .
$$

Proof. We begin by evaluating $\Delta D$. For any $K \in \mathscr{Y}_{l-1}$ we want to calculate $\mathbb{P}\left(\Delta\left(j_{l}(X) Y(l)\right) K\right)=\mathbb{P}\left(\Delta\left(U(l)^{*} X\left(A(l)+A^{*}(l)\right) U(l)\right) K\right)$. We proceed exactly as in the proof of Lemma 5.7. Using (5.6) and the quantum Itô rules, we obtain

$$
\begin{gathered}
\Delta\left(U(l)^{*} X\left(A(l)+A^{*}(l)\right) U(l)\right)=(\cdots) \Delta \Lambda(l)+(\cdots) \Delta A^{*}(l)+(\cdots) \Delta A(l) \\
+U(l-1)^{*} \mathcal{L}(X)\left(A(l-1)+A^{*}(l-1)\right) U(l-1) \Delta t(l) \\
\quad+j_{l-1}\left(X M^{+}+M^{+*} X+\lambda^{2} M^{\circ *} X M^{+}+\lambda^{2} M^{+*} X M^{\circ}\right) \Delta t(l) .
\end{gathered}
$$

It follows in the usual way that

$$
\begin{aligned}
& \Delta D(l)=\pi_{l-1}(\mathcal{L}(X)) Y(l-1) \Delta t(l) \\
& \quad+\pi_{l-1}\left(X M^{+}+M^{+*} X+\lambda^{2} M^{\circ *} X M^{+}+\lambda^{2} M^{+*} X M^{\circ}\right) \Delta t(l) .
\end{aligned}
$$

We now turn our attention to $\Delta E$. First note that the Itô rule gives

$$
\Delta\left(\pi_{l}(X) Y(l)\right)=\left(\Delta \pi_{l}(X)\right) Y(l-1)+\pi_{l-1}(X) \Delta Y(l)+\Delta \pi_{l}(X) \Delta Y(l) .
$$

By uniqueness of the Doob decomposition, $\Delta E$ is the sum of the predictable parts $\Delta E_{1,2,3}$ of the three terms on the right-hand side. Let us investigate each of these individually. The first term can be written as

$$
\left(\Delta \pi_{l}(X)\right) Y(l-1)=\pi_{l-1}(\mathcal{L}(X)) Y(l-1) \Delta t(l)+Y(l-1) \Delta H(l) .
$$

Copyright $@$ by SIAM. Unauthorized reproduction of this article is prohibited. 
It is easily verified, however, that $\sum_{l} Y(l-1) \Delta H(l)$ inherits the martingale property from $H$. Hence, by uniqueness of the Doob decomposition, we obtain $\Delta E_{1}(l)=$ $\pi_{l-1}(\mathcal{L}(X)) Y(l-1) \Delta t(l)$. Moving on to the second term, we write

$$
\pi_{l-1}(X) \Delta Y(l)=\pi_{l-1}(X) \Delta C(l)+\pi_{l-1}(X) \Delta \tilde{Y}(l) .
$$

Similarly to above we find that $\sum_{l} \pi_{l-1}(X) \Delta \tilde{Y}(l)$ inherits the martingale property from $\tilde{Y}$, so that evidently $\Delta E_{2}(l)=\pi_{l-1}(X) \Delta C(l)$ (where $\Delta C(l)$ is given explicitly in Lemma 5.7). It remains to deal with the third term. To this end, let us write

$$
\Delta \pi_{l}(X) \Delta Y(l)=\left\{\pi_{l-1}(\mathcal{L}(X)) \Delta t(l)+\Xi(l) \Delta \tilde{Y}(l)\right\}(\Delta C(l)+\Delta \tilde{Y}(l)) .
$$

As before, processes of the form $\sum_{l} X(l) \Delta \tilde{Y}(l)$ with $\mathscr{Y}$-predictable $X$ inherit the martingale property from $\tilde{Y}$. Thus we only need to retain the predictable terms:

$$
\Delta \pi_{l}(X) \Delta Y(l)=(\cdots) \Delta \tilde{Y}(l)+\pi_{l-1}(\mathcal{L}(X)) \Delta t(l) \Delta C(l)+\Xi(l)(\Delta \tilde{Y}(l))^{2} .
$$

Similarly, we expand $(\Delta \tilde{Y}(l))^{2}$ as

$$
(\Delta \tilde{Y}(l))^{2}=(\cdots) \Delta \tilde{Y}(l)+(\Delta Y(l))^{2}-(\Delta C(l))^{2},
$$

where we have used $\Delta Y(l)=\Delta C(l)+\Delta \tilde{Y}(l)$. But using the Itô rules we calculate $(\Delta Y(l))^{2}=U(l)^{*}\left(\Delta A(l)+\Delta A^{*}(l)\right)^{2} U(l)=\Delta t(l)$. Hence we can read off

$$
\Delta E_{3}(l)=\pi_{l-1}(\mathcal{L}(X)) \Delta t(l) \Delta C(l)+\Xi(l)\left(\Delta t(l)-(\Delta C(l))^{2}\right) .
$$

But recall that $j_{l}(X) Y(l)$ and $\pi_{l}(X) Y(l)$ have equal predictable parts. Hence setting $\Delta D(l)=\Delta E_{1}(l)+\Delta E_{2}(l)+\Delta E_{3}(l)$ and solving for $\Xi(l)$, the lemma follows.

Putting everything together we get the following discrete quantum filtering equation for homodyne detection:

$$
\begin{aligned}
& \Delta \pi_{l}(X)=\pi_{l-1}(\mathcal{L}(X)) \Delta t(l) \\
& \quad+\frac{\pi_{l-1}(\mathcal{J}(X))-\pi_{l-1}\left(X+\lambda^{2} \mathcal{L}(X)\right) \pi_{l-1}(\mathcal{J}(I))}{I-\lambda^{2} \pi_{l-1}(\mathcal{J}(I))^{2}}\left(\Delta Y(l)-\pi_{l-1}(\mathcal{J}(I)) \Delta t(l)\right) .
\end{aligned}
$$

5.3. Photodetection. We now turn our attention to a setup where we are counting photons in the field, i.e., we are observing

$$
Y^{\Lambda}(l)=U(l)^{*} \Lambda(l) U(l), \quad 0 \leq l \leq k .
$$

The procedure here is much the same as in the homodyne detection case.

Lemma 5.9. The predictable process $C$ in the decomposition of $Y^{\Lambda}$ is given by

$$
\Delta C(l)=\pi_{l-1}\left(M^{+*} M^{+}\right) \Delta t(l), \quad 1 \leq l \leq k .
$$

Proof. By (5.1), we have $\Delta C(l)=\mathbb{P}\left(\Delta Y^{\Lambda}(l) \mid \mathscr{Y}_{l-1}\right)$. To calculate the latter, let $K$ be an element in $\mathscr{Y}_{l-1}$; we would like find an expression for $\mathbb{P}\left(K \Delta Y^{\Lambda}(l)\right)$. To this end, we calculate using the discrete quantum Itô rules that

$$
\begin{aligned}
\Delta(\Lambda(l) U(l)) & =(\cdots) \Delta \Lambda(l)+(\cdots) \Delta A(l) \\
+ & (I+\Lambda(l-1)) M^{+} U(l-1) \Delta A^{*}(l)+\Lambda(l-1) M^{\circ} U(l-1) \Delta t(l),
\end{aligned}
$$

Copyright $@$ by SIAM. Unauthorized reproduction of this article is prohibited. 
where we have retained the relevant terms. Consequently, we calculate

$$
\begin{aligned}
\Delta\left(U(l)^{*} \Lambda(l) U(l)\right) & =(\cdots) \Delta \Lambda(l)+(\cdots) \Delta A^{*}(l)+(\cdots) \Delta A(l) \\
& +U(l-1)^{*} \mathcal{L}(I) \Lambda(l-1) U(l-1) \Delta t(l)+j_{l-1}\left(M^{+*} M^{+}\right) \Delta t(l),
\end{aligned}
$$

where $\mathcal{L}(X)$ is the discrete Lindblad generator. But $\mathcal{L}(I)$ vanishes, and the lemma follows by the usual argument.

Next, we determine the predictable process $\Xi(l)$ such that $\Delta H(l)=\Xi(l) \Delta \tilde{Y}(l)$.

LEMMA 5.10. The process $\Xi(l)$ is given by

$$
\Xi(l)=\left(I-\lambda^{2} \pi_{l-1}\left(M^{+*} M^{+}\right)\right)^{-1}\left[\frac{\pi_{l-1}\left(M^{+*} X M^{+}\right)}{\pi_{l-1}\left(M^{+*} M^{+}\right)}-\pi_{l-1}\left(X+\lambda^{2} \mathcal{L}(X)\right)\right] .
$$

Proof. We begin by finding the predictable process $\Delta D$ in the Doob decomposition of $j_{l}(X) Y(l)$. For any $K \in \mathscr{Y}_{l-1}$ we want to calculate $\mathbb{P}\left(\Delta\left(j_{l}(X) Y(l)\right) K\right)=$ $\mathbb{P}\left(\Delta\left(U(l)^{*} X \Lambda(l) U(l)\right) K\right)$. Using (5.7) and the quantum Itô rules, we obtain

$$
\begin{aligned}
& \Delta\left(U(l)^{*} X \Lambda(l) U(l)\right)=(\cdots) \Delta \Lambda(l)+(\cdots) \Delta A^{*}(l)+(\cdots) \Delta A(l) \\
& +U(l-1)^{*} \mathcal{L}(X) \Lambda(l-1) U(l-1) \Delta t(l)+j_{l-1}\left(M^{+*} X M^{+}\right) \Delta t(l) .
\end{aligned}
$$

It follows in the usual way that

$$
\Delta D(l)=\pi_{l-1}(\mathcal{L}(X)) Y(l-1) \Delta t(l)+\pi_{l-1}\left(M^{+*} X M^{+}\right) \Delta t(l) .
$$

We now turn our attention to the predictable process $\Delta E$ in the Doob decomposition of $\pi_{l}(X) Y_{l}$. First note that the Itô rule gives

$$
\Delta\left(\pi_{l}(X) Y(l)\right)=\left(\Delta \pi_{l}(X)\right) Y(l-1)+\pi_{l-1}(X) \Delta Y(l)+\Delta \pi_{l}(X) \Delta Y(l) .
$$

By uniqueness of the Doob decomposition, $\Delta E$ is the sum of the predictable parts $\Delta E_{1,2,3}$ of the three terms on the right-hand side. As in the proof of Lemma 5.8, we find that $\Delta E_{1}(l)=\pi_{l-1}(\mathcal{L}(X)) Y(l-1) \Delta t(l)$ and $\Delta E_{2}(l)=\pi_{l-1}(X) \Delta C(l)$ (where $\Delta C(l)$ is given explicitly in Lemma 5.9). To deal with the third term, we write

$$
\Delta \pi_{l}(X) \Delta Y(l)=\left\{\pi_{l-1}(\mathcal{L}(X)) \Delta t(l)+\Xi(l) \Delta \tilde{Y}(l)\right\}(\Delta C(l)+\Delta \tilde{Y}(l)) .
$$

As before, processes of the form $\sum_{l} X(l) \Delta \tilde{Y}(l)$ with $\mathscr{Y}$-predictable $X$ inherit the martingale property from $\tilde{Y}$. Thus we only need to retain the predictable terms:

$$
\Delta \pi_{l}(X) \Delta Y(l)=(\cdots) \Delta \tilde{Y}(l)+\pi_{l-1}(\mathcal{L}(X)) \Delta t(l) \Delta C(l)+\Xi(l)(\Delta \tilde{Y}(l))^{2} .
$$

Similarly, we expand $(\Delta \tilde{Y}(l))^{2}$ as

$$
(\Delta \tilde{Y}(l))^{2}=(\cdots) \Delta \tilde{Y}(l)+(\Delta Y(l))^{2}-(\Delta C(l))^{2},
$$

where we have used $\Delta Y(l)=\Delta C(l)+\Delta \tilde{Y}(l)$. But using the Itô rules we calculate $(\Delta Y(l))^{2}=U(l)^{*} \Delta \Lambda(l)^{2} U(l)=\Delta Y(l)$. Hence

$$
(\Delta \tilde{Y}(l))^{2}=(\cdots) \Delta \tilde{Y}(l)+\Delta C(l)-(\Delta C(l))^{2},
$$

and we can see that

$$
\Delta E_{3}(l)=\pi_{l-1}(\mathcal{L}(X)) \Delta t(l) \Delta C(l)+\Xi(l)(I-\Delta C(l)) \Delta C(l) .
$$

Copyright $@$ by SIAM. Unauthorized reproduction of this article is prohibited. 
But recall that $j_{l}(X) Y(l)$ and $\pi_{l}(X) Y(l)$ have equal predictable parts. Hence setting $\Delta D(l)=\Delta E_{1}(l)+\Delta E_{2}(l)+\Delta E_{3}(l)$ and solving for $\Xi(l)$, the lemma follows.

Putting everything together we obtain the following discrete quantum filtering equation for photon counting:

$$
\begin{aligned}
& \Delta \pi_{l}(X)=\pi_{l-1}(\mathcal{L}(X)) \Delta t(l)+\left(I-\lambda^{2} \pi_{l-1}\left(M^{+*} M^{+}\right)\right)^{-1} \\
& \quad \times\left[\frac{\pi_{l-1}\left(M^{+*} X M^{+}\right)}{\pi_{l-1}\left(M^{+*} M^{+}\right)}-\pi_{l-1}\left(X+\lambda^{2} \mathcal{L}(X)\right)\right]\left(\Delta Y(l)-\pi_{l-1}\left(M^{+*} M^{+}\right) \Delta t(l)\right) .
\end{aligned}
$$

5.4. How to Use the Filtering Equations. The filtering equations of sections 5.2 and 5.3 may seem a little abstract at this point; $\pi_{l}(X)$ is some observable in the algebra $\mathscr{M} \otimes \mathscr{W}_{k}$, and it appears that we would need to know $\pi_{l-1}(Z)$ for every $Z \in \mathscr{B}_{0}$, in addition to the observation increment $\Delta Y(l)$, in order to be able to calculate $\pi_{l}(X)$ for arbitrary $X$. The equations are much less abstract than they might appear, however. First of all, recall that both $\pi_{l}(X)$ and $\Delta Y(l)$ are elements of the (commutative) observation algebra $\mathscr{Y}_{k}$; in fact, the filtering equations live entirely within this algebra. Hence these are just classical equations in disguise (as they should be!); we could write explicitly, e.g., in the homodyne detection case,

$$
\begin{aligned}
\Delta \iota\left(\pi_{l}(X)\right)=\iota\left(\pi_{l-1}(\mathcal{L}(X))\right) \lambda^{2}+\left(\iota(\Delta Y(l))-\iota\left(\pi_{l-1}(\mathcal{J}(I))\right) \lambda^{2}\right) \\
\times \frac{\iota\left(\pi_{l-1}(\mathcal{J}(X))\right)-\iota\left(\pi_{l-1}\left(X+\lambda^{2} \mathcal{L}(X)\right)\right) \iota\left(\pi_{l-1}(\mathcal{J}(I))\right)}{1-\lambda^{2} \iota\left(\pi_{l-1}(\mathcal{J}(I))\right)^{2}}
\end{aligned}
$$

using the ${ }^{*}$-isomorphism $\iota$ obtained by applying the spectral theorem to $\left(\mathscr{Y}_{k}, \mathbb{P}\right)$. For any $0 \leq l \leq k$ and $X \in \mathscr{M}, \iota\left(\pi_{l}(X)\right)$ is a random variable that is a function of the random process $\iota(\Delta Y(i))$ up to and including time $l$. But an elementary property of the conditional expectation is that $\pi_{l}(X)$ is linear in $X$; hence $\iota\left(\pi_{l}(X)\right)$ is also linear in $X$. This means we can always write $\iota\left(\pi_{l}(X)\right)=\operatorname{Tr}\left[\rho_{l} X\right]$, where $\rho_{l}$ is a (random) $2 \times 2$ matrix (as $\mathscr{M}$ is two-dimensional). We obtain the following recursion for $\rho_{l}$ :

$$
\begin{aligned}
& \Delta \rho_{l}=\overline{\mathcal{L}}\left(\rho_{l-1}\right) \lambda^{2}+\frac{\overline{\mathcal{J}}\left(\rho_{l-1}\right)-\operatorname{Tr}\left[\overline{\mathcal{J}}\left(\rho_{l-1}\right)\right]\left(\rho_{l-1}+\lambda^{2} \overline{\mathcal{L}}\left(\rho_{l-1}\right)\right)}{1-\lambda^{2} \operatorname{Tr}\left[\overline{\mathcal{J}}\left(\rho_{l-1}\right)\right]^{2}} \\
& \times\left(\iota(\Delta Y(l))-\operatorname{Tr}\left[\overline{\mathcal{J}}\left(\rho_{l-1}\right)\right] \lambda^{2}\right),
\end{aligned}
$$

where we have written

$$
\begin{aligned}
& \overline{\mathcal{L}}(\rho)=M^{+} \rho M^{+*}+\lambda^{2} M^{\circ} \rho M^{\circ *}+M^{\circ} \rho+\rho M^{\circ *}, \\
& \overline{\mathcal{J}}(\rho)=M^{+} \rho+\rho M^{+*}+\lambda^{2} M^{+} \rho M^{\circ *}+\lambda^{2} M^{\circ} \rho M^{+*} .
\end{aligned}
$$

$\rho_{0}$ is simply the density matrix corresponding to the initial state $\rho(\cdot)$, i.e., $\rho(X)=$ $\operatorname{Tr}\left[\rho_{0} X\right]$ for every $X \in \mathscr{M}$. The matrix $\rho_{l}$ is called the conditional density matrix and contains all the information needed to calculate $\pi_{l}(X)$ for every $X \in \mathscr{M}$. Furthermore, (5.9) is a simple nonlinear recursion for $2 \times 2$ matrices. At any time step $l$ we only need to remember the $2 \times 2$ matrix $\rho_{l}$; when the $(l+1)$ th observation $\iota(\Delta Y(l+1))$ becomes available, which takes one of the values $\pm \lambda$, we simply plug this value into (5.9) and obtain the updated matrix $\rho_{l+1}$. Such a recursion is very efficient and, if necessary, would be easily implemented on a digital signal processor.

As filtering equations are entirely classical, there is no real need to make the explicit distinction between their representation in terms of classical random variables on a probability space vs. elements of the observation algebra. We urge the reader 
to always think of sets of commuting observables as random variables; this is implied by the spectral theorem, and is at the heart of quantum mechanics! Equation (5.8) is notationally tedious and completely unnecessary, as it does not add anything to the filtering equation as we have already written it in section 5.2. In some cases, e.g., in the proof of the martingale representation theorem, it is convenient to use explicitly the structure of the underlying probability space, but in much of this article we will not make an explicit distinction between random variables and observables.

A similar story holds for the photodetection case; we leave it up to the reader to calculate the associated recursion for the conditional density matrix.

5.5. The Markov Property and Monte Carlo Simulations. The filtering equations that we have developed take as input the observation process obtained from the system. Though this is precisely how it should be, one would think that further investigation of the filters could not proceed without the availability of typical sample paths of the observations from some other source, be it an actual physical system or a direct computer simulation of the underlying repeated interaction model. It is thus somewhat surprising that we can actually simulate such sample paths using the filtering equation only, without any auxiliary input. This is due to the Markov property of the filter, which we will demonstrate shortly. We can use this property of the filtering equations to perform Monte Carlo simulations of both the sample paths of the observation process and sample paths of the filter itself (called "quantum trajectories" in the physics literature). In addition, the Markov property is key for the development of feedback controls, as we will see in sections 7-9.

We will consider below the homodyne detection case, but the photodetection case proceeds identically. Set $\Delta y_{l}=\iota(\Delta Y(l))$. Recall that the homodyne detection signal $\Delta y_{l}$ takes one of two values $\pm \lambda$ for every $l$. Suppose we have observed $y_{i}$ up to and including time $l-1$; we would like to be able to calculate the probability distribution of $\Delta y_{l}$ using this information. This calculation is carried out in the following lemma.

LEMMA 5.11. We have

$$
\mathbf{P}\left[\Delta y_{l}= \pm \lambda \mid \mathcal{Y}_{l-1}\right]=p\left(\Delta y_{l}= \pm \lambda ; \rho_{l-1}\right),
$$

where

$$
p(\Delta y= \pm \lambda ; \rho)=\frac{1}{2} \pm \frac{\lambda}{2} \operatorname{Tr}[\overline{\mathcal{J}}(\rho)]
$$

depends only on the filter in the previous time step.

Proof. Let $p_{l}^{+}=\mathbf{P}\left[\Delta y_{l}= \pm \lambda \mid \mathcal{Y}_{l-1}\right]$ be the probability that the observation in the next time step $\Delta y_{l}$ takes the value $+\lambda$. Using the Doob decomposition we have

$$
\Delta Y(l)=\Delta C(l)+\Delta \tilde{Y}(l)=\pi_{l-1}(\mathcal{J}(I)) \Delta t(l)+\Delta \tilde{Y}(l),
$$

where $\Delta \tilde{Y}(l)$ is a martingale increment, so that

$$
\lambda p_{l}^{+}-\lambda\left(1-p_{l}^{+}\right)=\iota\left(\mathbb{P}\left(\Delta Y(l) \mid \mathscr{Y}_{l-1}\right)\right)=\iota\left(\pi_{l-1}(\mathcal{J}(I))\right) \lambda^{2} .
$$

Thus

$$
p_{l}^{+}=\frac{1}{2}+\frac{\lambda}{2} \operatorname{Tr}\left[\rho_{l-1} \mathcal{J}(I)\right]=\frac{1}{2}+\frac{\lambda}{2} \operatorname{Tr}\left[\bar{J}\left(\rho_{l-1}\right)\right]
$$

depends only on the filter in the previous time step. 
With this distribution in hand, we can also calculate the statistics of the filter in the next time step, and we can prove the Markov property by recursing this procedure.

Lemma 5.12. The filter $\rho_{l}$ satisfies the following Markov property:

$$
\mathbf{E}_{\mathbf{P}}\left(g\left(\rho_{j}\right) \mid \sigma\left\{\rho_{0}, \ldots, \rho_{l}\right\}\right)=\mathbf{E}_{\mathbf{P}}\left(g\left(\rho_{j}\right) \mid \sigma\left\{\rho_{l}\right\}\right) \quad \forall l \leq j \leq k .
$$

Proof. Using the recursion (5.9), $\rho_{j}$ can be written as a deterministic function $f\left(\rho_{j-1}, \Delta y_{j}\right)$ of $\rho_{j-1}$ and $\Delta y_{j}$. By the martingale property of the innovation process,

$$
\mathbf{P}\left(\Delta y_{j}= \pm \lambda \mid \sigma\left\{y_{1}, \ldots, y_{j-1}\right\}\right)=\frac{1}{2} \pm \frac{\lambda}{2} \operatorname{Tr}\left[\overline{\mathcal{J}}\left(\rho_{j-1}\right)\right],
$$

which is only a function of $\rho_{j-1}$. As $\sigma\left\{\rho_{0}, \ldots, \rho_{j-1}\right\} \subset \sigma\left\{y_{1}, \ldots, y_{j-1}\right\}$, we obtain

$$
\mathbf{P}\left(\Delta y_{j}= \pm \lambda \mid \sigma\left\{\rho_{0}, \ldots, \rho_{j-1}\right\}\right)=\frac{1}{2} \pm \frac{\lambda}{2} \operatorname{Tr}\left[\overline{\mathcal{J}}\left(\rho_{j-1}\right)\right] .
$$

Hence for any function $g$

$$
\begin{aligned}
& \mathbf{E}_{\mathbf{P}}\left(g\left(\rho_{j}\right) \mid \sigma\left\{\rho_{0}, \ldots, \rho_{j-1}\right\}\right)=\mathbf{E}_{\mathbf{P}}\left(g^{\prime}\left(\rho_{j-1}, \Delta y_{j}\right) \mid \sigma\left\{\rho_{0}, \ldots, \rho_{j-1}\right\}\right) \\
&=\sum_{a \in\{-1,1\}} g^{\prime}\left(\rho_{j-1}, a \lambda\right)\left[\frac{1}{2}+a \frac{\lambda}{2} \operatorname{Tr}\left[\overline{\mathcal{J}}\left(\rho_{j-1}\right)\right]\right],
\end{aligned}
$$

where we have written $g^{\prime}(\rho, w)=g(f(\rho, w))$ for the function $g$ composed with the one-step filter recursion. As the right-hand side is a function of $\rho_{j-1}$ only, we have

$$
\mathbf{E}_{\mathbf{P}}\left(g\left(\rho_{j}\right) \mid \sigma\left\{\rho_{0}, \ldots, \rho_{j-1}\right\}\right)=\mathbf{E}_{\mathbf{P}}\left(\mathbf{E}_{\mathbf{P}}\left(g\left(\rho_{j}\right) \mid \sigma\left\{\rho_{0}, \ldots, \rho_{j-1}\right\}\right) \mid \sigma\left\{\rho_{j-1}\right\}\right),
$$

from which we conclude that

$$
\mathbf{E}_{\mathbf{P}}\left(g\left(\rho_{j}\right) \mid \sigma\left\{\rho_{0}, \ldots, \rho_{j-1}\right\}\right)=\mathbf{E}_{\mathbf{P}}\left(g\left(\rho_{j}\right) \mid \sigma\left\{\rho_{j-1}\right\}\right) .
$$

But setting $\mathbf{E}_{\mathbf{P}}\left(g\left(\rho_{j}\right) \mid \sigma\left\{\rho_{0}, \ldots, \rho_{j-1}\right\}\right)=h\left(\rho_{j-1}\right)$, we can repeat the argument giving

$$
\mathbf{E}_{\mathbf{P}}\left(h\left(\rho_{j-1}\right) \mid \sigma\left\{\rho_{0}, \ldots, \rho_{j-2}\right\}\right)=\mathbf{E}_{\mathbf{P}}\left(h\left(\rho_{j-1}\right) \mid \sigma\left\{\rho_{j-2}\right\}\right) .
$$

From the definition of $h(\cdot)$ we immediately obtain

$$
\mathbf{E}_{\mathbf{P}}\left(g\left(\rho_{j}\right) \mid \sigma\left\{\rho_{0}, \ldots, \rho_{j-2}\right\}\right)=\mathbf{E}_{\mathbf{P}}\left(g\left(\rho_{j}\right) \mid \sigma\left\{\rho_{j-2}\right\}\right) .
$$

Recursing the argument gives the Markov property.

It is now straightforward to turn this procedure into a Monte Carlo algorithm. The following pseudocode generates random sample paths of the observations $\Delta y_{l}$ and filter $\rho_{l}$, sampled faithfully from the probability measure induced by the repeated interaction model on the space of observation paths.

1. Initialize $\rho_{0}$.

2. $l \leftarrow 0$.

3. REPEAT

(A) Calculate $p_{l+1}^{+}\left(\rho_{l}\right)$.

(B) SAMPle $\xi \sim$ UNIFORm $[0,1]$.

(C) IF $\xi<p_{l+1}^{+}: \Delta y_{l+1} \leftarrow+\lambda$; ELSE: $\Delta y_{l+1} \leftarrow-\lambda$.

(D) $\rho_{l+1} \leftarrow \rho_{l}+\Delta \rho_{l+1}\left(\rho_{l}, \Delta y_{l+1}\right)$.

(E) $l \leftarrow l+1$

4. UNTIL $l=k$.

Copyright (c) by SIAM. Unauthorized reproduction of this article is prohibited. 

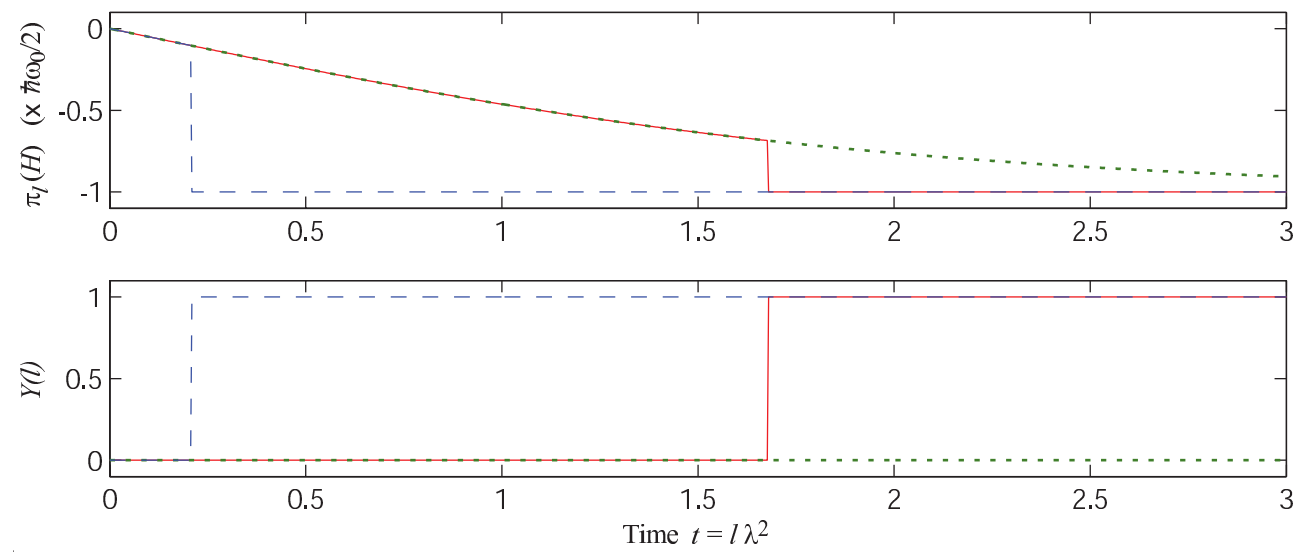

Fig. 5.I (Spontaneous emission.) Three typical sample paths of the conditional expectation $\pi_{l}(H)$ of the atomic energy (top plot) with respect to the integrated photodetection signal $Y(l)$ (bottom plot). The atom spontaneously emits exactly one photon at a random time, after which it attains its lowest energy and remains there. The initial state chosen was $\rho(x)=$ $\operatorname{Tr}[X] / 2$; in this state $H= \pm \hbar \omega_{0} / 2$ have equal probability. The time scale used for the calculation is $\lambda^{-2}=300$.

5.6. Examples. Using the Monte Carlo method developed in the previous section, we can now simulate the observation and filter sample paths for our usual examples. In the simulations we have used the initial state $\rho(X)=\operatorname{Tr}[X] / 2$, under which the probabilities of the energy attaining its maximal or minimal values are equal.

Spontaneous Emission. In Figure 5.1, photodetection of a spontaneously emitting atom is simulated. The observation process takes a very simple form: if the atom initially attained its maximal energy (which it does with probability $\frac{1}{2}$ under the state $\rho$ ), it emits a single photon at a random time. If the atom was already at its lowest energy (also with probability $\frac{1}{2}$ ), the atom never emits a photon. The conditional expectation of the energy attains its minimal value immediately after the observation of a photon, as at this point we know that the atom has attained its lowest energy. Before the observation of a photon, the conditional expectation decays: the longer we fail to observe a photon, the higher the (conditional) probability that the atom started out with minimal energy to begin with.

Note that the higher the initial expectation of the energy of the atom, the slower the decay of $\pi_{l}(H)$; after all, if the probability of the atom starting out at its minimal energy is very small, then we should fail to observe a photon for a very long time before concluding that the atom, against all odds, did start off with minimal energy. In the extreme case of unit initial probability that the atom has maximal energy $\left(\rho(X)=\operatorname{Tr}\left[\sigma_{+} \sigma_{-} X\right]\right)$, the conditional expectation of the energy is a step function. The latter can be verified directly using the filtering equation for photodetection, which shows that $\Delta \pi_{l}(H)=0$ as long as $\Delta Y(l)=0$ and $\pi_{l-1}(H)=\hbar \omega_{0} / 2$.

Figure 5.2 shows a simulation of the same system, but now observed using a homodyne detection setup. Evidently the way in which information on the atomic energy is encoded in the homodyne observations is very different than in the photodetection case; rather than the sudden gain of information when a photon is observed, homodyne detection allows us to gradually infer the atomic energy from the noisy measurements. The observation process itself is not very revealing to the naked eye, 

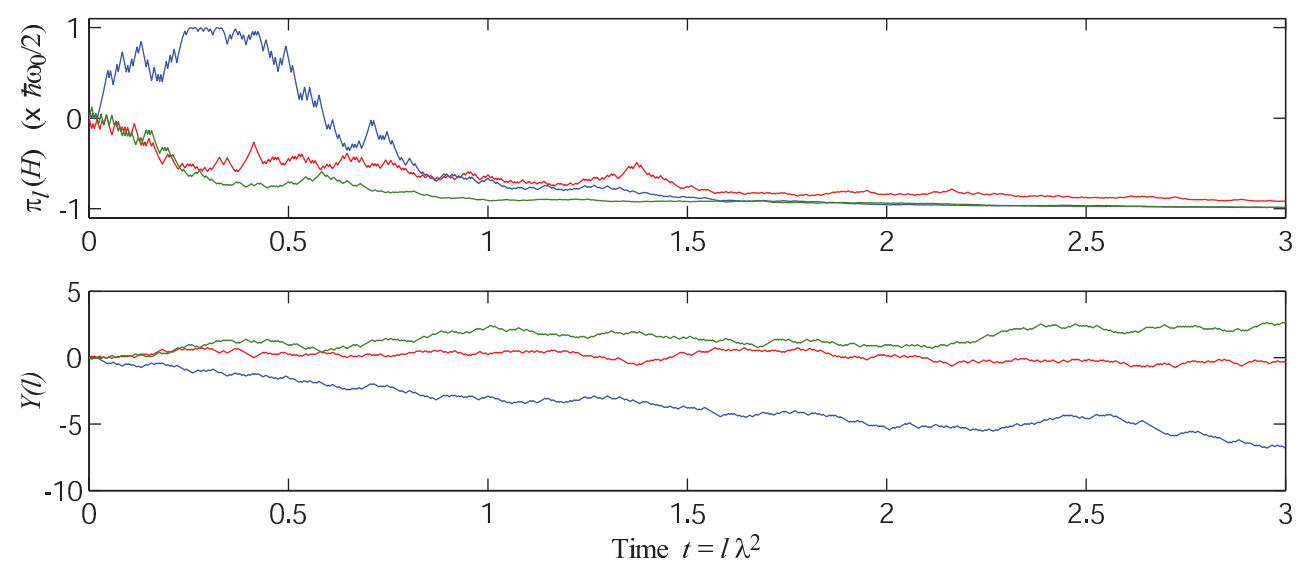

Fig. 5.2 (Spontaneous emission.) Three typical sample paths of the conditional expectation $\pi_{l}(H)$ of the atomic energy (top plot) with respect to the integrated homodyne photocurrent $Y(l)$ (bottom plot). It is difficult to infer much about the atomic energy from the observation process using the naked eye, but nonetheless the conditional expectation is revealing (e.g., in the case of the blue sample path, the atom initially possessed maximal energy with unit probability). The initial state chosen was $\rho(x)=\operatorname{Tr}[X] / 2$, and the time scale used for the calculation is $\lambda^{-2}=300$.

but the filter manages to make sense of it. In the case of the blue curve, for example, we infer that the atom almost certainly attained its maximal energy value before time $t \sim 0.5$, whereas after time $t \sim 1.5$ all three paths indicate that most likely the atom has attained its lowest energy.

Dispersive Interaction. The dispersive interaction case is quite different than the spontaneous emission case. Recall that in this case the energy observable $j_{l}(H)=H$ is constant in time. This does not mean, however, that the conditional expectation $\pi_{l}(H)$ is constant. Whether the energy attains its maximal or minimal value in a particular realization determines the mean direction of the phase shift of the outgoing light, which can be measured using a homodyne detection setup. As we gain information on the atomic energy, the conditional expectation is gradually attracted to the value actually taken by the energy $H$ in that realization.

This behavior is demonstrated in the simulation of Figure 5.3. Each of the filter sample paths $\pi_{l}(H)$ is attracted to either $+\hbar \omega_{0} / 2$ or $-\hbar \omega_{0} / 2$. As the probabilities of maximal and minimal atomic energy are equal under the initial state $\rho(X)=\operatorname{Tr}[X] / 2$, the sample paths of $\pi_{l}(H)$ are attracted to $\pm \hbar \omega_{0} / 2$ with equal probability. It would be presumptuous to conclude this from the simulation of only three sample paths, but if we are willing to believe that $\pi_{l}(H) \rightarrow \pm \hbar \omega_{0} / 2$ with unit probability, then the result is evident: after all, $\mathbb{P}\left(\pi_{l}(H)\right)=\mathbb{P}\left(j_{l}(H)\right)=\rho(H)=0$ for all $l$, so we can only have $\pi_{l}(H) \rightarrow \pm \hbar \omega_{0} / 2$ if the two possibilities occur with equal probability (the probabilities change accordingly if we choose a different initial state $\rho$ ). The fact that $\pi_{l}(H) \rightarrow \pm \hbar \omega_{0} / 2$ with unit probability can also be rigorously proved, but we will postpone this discussion until section 9 .

Note that the behavior of the filter can already be seen by inspecting the observation process using the naked eye: though the observation processes are still random, the integrated observations have an upward or downward trend depending on the value of the atomic energy. This indicates that the actual observation process $\Delta Y(l)$ 

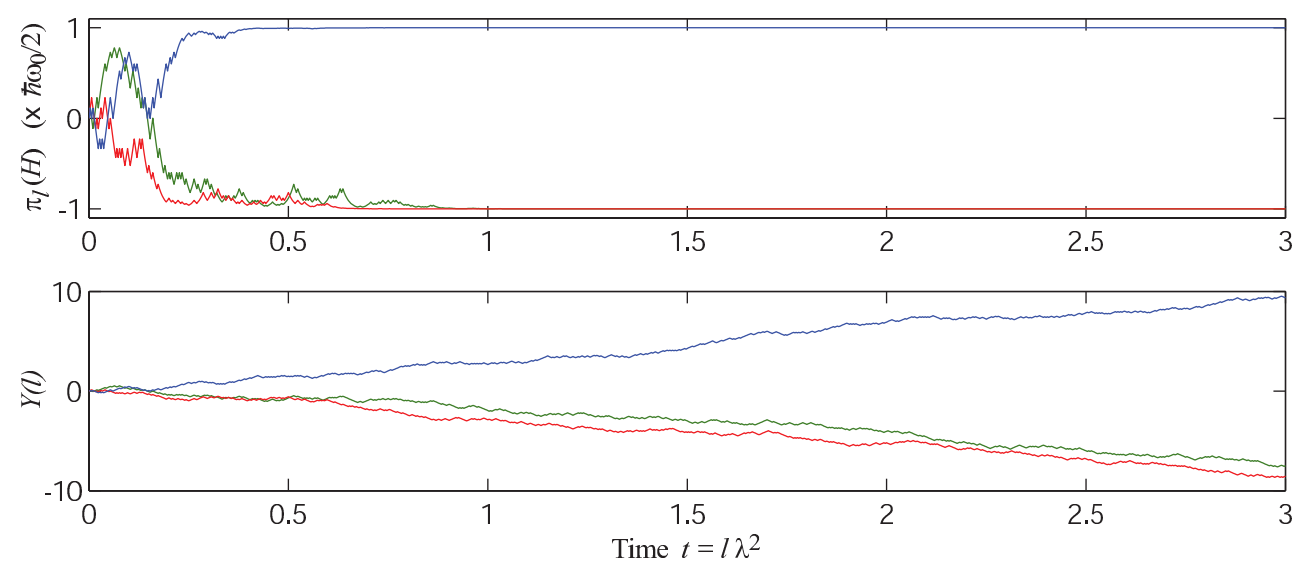

Fig. 5.3 (Dispersive interaction.) Three typical sample paths of the conditional expectation $\pi_{l}(H)$ of the atomic energy (top plot) with respect to the integrated homodyne photocurrent $Y(l)$ (bottom plot). Though the energy observable is constant in time, the observations contain information on the atomic energy so that the conditional expectation converges to the actual value of the energy. The initial state chosen was $\rho(x)=\operatorname{Tr}[X] / 2$, and the time scale used is $\lambda^{-2}=300$.

is positive (negative) on average if the atomic energy is positive (negative), i.e., positive atomic energy leads to an average positive phase shift on the output light, whereas negative atomic energy gives rise to a negative average phase shift.

We have not shown a simulation of the dispersively interacting atom under photodetection. Though many photons can be observed, a photodetector gives no information on the phase of the output light. Hence no information is gained about the atomic energy, and the conditional expectation of the atomic energy is constant. Evidently the type of detector used makes a big difference in this case.

6. The Reference Probability Approach. In the previous section we obtained explicit nonlinear recursive equations for the conditional expectations $\pi_{l}(X)$. In this section we start from scratch and solve the filtering problem in an entirely different way. The key idea here is that the Bayes Lemma 3.4 allows us to express the filtering problem in terms of an arbitrary (reference) state; by choosing the "Radon-Nikodým" operator $V$ conveniently, we can reduce the calculation to a manageable form. This gives rise to a linear recursion for the numerator in the Bayes formula, which we denote by $\sigma_{l}(X) . \pi_{l}(X)$ is then calculated as $\sigma_{l}(X) / \sigma_{l}(I)$.

The reference probability method is widely used in classical filtering theory following the work of Duncan [32], Mortensen [70], Zakai [88], and Kallianpur and Striebel $[56,57]$. See, e.g., [34] for a systematic exposition of reference probability methods in filtering and control. The corresponding approach in quantum filtering theory, adapted below to the discrete setting, was developed in [17].

6.I. The Strategy. Let us begin by outlining what we are going to do. We are looking for a way to calculate $\pi_{l}(X)=\mathbb{P}\left(j_{l}(X) \mid \mathscr{Y}_{l}\right)$. In classical filtering theory it has proved advantageous to express the problem, using the Bayes formula, in terms of a measure under which the signal (here the atomic observable $j_{l}(X)$ ) and the observations (here $\mathscr{Y}_{l}$ ) are independent. We will aim to do the same in the quantum case. Unlike in classical probability, however, we do not have a suitable quantum 
version of Girsanov's theorem to help us find such a change of measure. We need to use a little intuition to obtain the required change-of-state operator $V$.

The following simple lemma will make this task a little easier.

Lemma 6.1. Let $(\mathscr{A}, \mathbb{P})$ be a quantum probability space, $\mathscr{C} \subset \mathscr{A}$ a commutative subalgebra, and let $U \in \mathscr{A}$ be unitary. Define the rotated state $\mathbb{Q}(X)=\mathbb{P}\left(U^{*} X U\right)$ on $\mathscr{A}$. Then $\mathbb{P}\left(U^{*} X U \mid U^{*} \mathscr{C} U\right)=U^{*} \mathbb{Q}(X \mid \mathscr{C}) U$ for any $X \in \mathscr{C}^{\prime}$.

Proof. This is a simple consequence of the definition of conditional expectations. Let $K \in U^{*} \mathscr{C} U$. Then $\mathbb{P}\left(U^{*} \mathbb{Q}(X \mid \mathscr{C}) U K\right)=\mathbb{Q}\left(\mathbb{Q}(X \mid \mathscr{C}) U K U^{*}\right)=\mathbb{Q}\left(X U K U^{*}\right)=$ $\mathbb{P}\left(U^{*} X U K\right)$. But as this holds for any $K \in U^{*} \mathscr{C} U$ and as $U^{*} \mathbb{Q}(X \mid \mathscr{C}) U \in U^{*} \mathscr{C} U$, the lemma follows from the definition of the conditional expectation.

How does this help us in our usual setting $\left(\mathscr{M} \otimes \mathscr{W}_{k}, \mathbb{P}\right)$ ? Recall from section 2.5 that $\mathscr{C}_{l}=\operatorname{alg}\{\Delta Z(i): i=1, \ldots, l\}\left(Z=I \otimes \Lambda\right.$ or $Z=I \otimes\left(A+A^{*}\right)$ for photodetection or homodyne detection, respectively) and $\mathscr{Y}_{l}=U(l)^{*} \mathscr{C}_{l} U(l)$. For the time being, let us fix a time step $l$ and define the state $\mathbb{Q}^{l}(X)=\mathbb{P}\left(U(l)^{*} X U(l)\right)$. Then by Lemma 6.1 , we can write $\pi_{l}(X)=U(l)^{*} \mathbb{Q}^{l}\left(X \mid \mathscr{C}_{l}\right) U(l)$. Now note that $X \in \mathscr{B}_{0}$ is of the form $X \otimes I$ in $\mathscr{M} \otimes \mathscr{W}_{k}$, whereas every element of $\mathscr{C}_{l}$ is of the form $I \otimes C$. But we already know a state under which the initial system and the field are independent: this is simply the state $\mathbb{P}$ ! Hence if we could write $\mathbb{Q}^{l}(X)=\mathbb{P}\left(V(l)^{*} X V(l)\right)$ for some $V(l) \in \mathscr{C}_{l}^{\prime}$, then we would obtain using the Bayes Lemma 3.4

$$
\pi_{l}(X)=U(l)^{*} \mathbb{Q}^{l}\left(X \mid \mathscr{C}_{l}\right) U(l)=\frac{U(l)^{*} \mathbb{P}\left(V(l)^{*} X V(l) \mid \mathscr{C}_{l}\right) U(l)}{U(l)^{*} \mathbb{P}\left(V(l)^{*} V(l) \mid \mathscr{C}_{l}\right) U(l)} \quad \forall X \in \mathscr{B}_{0} .
$$

Note that we already have by construction $\mathbb{Q}^{l}(X)=\mathbb{P}\left(U(l)^{*} X U(l)\right)$, but $U(l) \notin \mathscr{C}_{l}^{\prime}$. Hence $V(l)=U(l)$ in the Bayes formula does not work. As we will demonstrate below, however, there is a simple trick which we can use to "push" $U(l)$ into the commutant $\mathscr{C}_{l}^{\prime}$ without changing the state $\mathbb{Q}^{l}$. This gives the desired change of state $V(l)$.

We emphasize that Lemma 6.1 is not an essential part of the procedure; we could try to find a new reference state $\mathbb{S}\left(V^{*} X V\right)=\mathbb{P}(X)$ and apply the Bayes lemma directly to $\mathbb{P}\left(j_{l}(X) \mid \mathscr{Y}_{l}\right)$ (in fact, the state $\mathbb{S}$ can be deduced from (6.1) by applying Lemma 6.1 in the reverse direction). The state $\mathbb{P}$ is a very convenient reference state, however, as it allows us to use the natural tensor splitting $\mathscr{M} \otimes \mathscr{W}_{k}$ and properties of the vacuum state $\phi^{\otimes k}$ to determine the necessary $V(l)$ with minimal effort. It is possible that this procedure could be streamlined in a more general theory for changes of state (in the spirit of the techniques widely used in classical probability theory), but such a theory is not currently available in quantum probability.

6.2. Homodyne Detection. We first consider the homodyne detection case, i.e., $Z(l)=A(l)+A^{*}(l)$. To use (6.1), we are looking for $V(l) \in \mathscr{C}_{l}^{\prime}$ such that $\mathbb{Q}^{l}(X)=$ $\mathbb{P}\left(V(l)^{*} X V(l)\right)$. The following trick [45] allows us to find such a $V(l)$ simply by modifying the quantum stochastic difference equation for $U(l)$; see (4.1).

LEMMA 6.2. Let $V$ be the solution of the following difference equation:

$$
\Delta V(l)=\left\{M^{+}\left(\Delta A(l)+\Delta A^{*}(l)\right)+M^{\circ} \Delta t(l)\right\} V(l-1), \quad V(0)=I .
$$

Then $V(l) \in \mathscr{C}_{l}^{\prime}$ and $\mathbb{P}\left(V(l)^{*} X V(l)\right)=\mathbb{P}\left(U(l)^{*} X U(l)\right)$ for any $X \in \mathscr{M} \otimes \mathscr{W}_{k}$.

Proof. The proof relies on the fact that $\mathbb{P}=\rho \otimes \phi^{\otimes k}$. For simplicity, let us assume that $\rho$ is a vector state, i.e., $\rho(X)=\langle v, X v\rangle$ for some $v \in \mathbb{C}^{2}$ (we will relax this requirement later on). Then $\mathbb{P}(X)=\left\langle v \otimes \Phi^{\otimes k}, X v \otimes \Phi^{\otimes k}\right\rangle$, where $\Phi$ is the vacuum vector. The essential property we need is that $\Delta A(l) \Phi^{\otimes k}=\Delta \Lambda(l) \Phi^{\otimes k}=0$; this follows immediately from their definitions (as $\Delta A(l) \Phi^{\otimes k}=\Phi^{\otimes l-1} \otimes \sigma_{-} \Phi \otimes \Phi^{\otimes k-l}=0$, 
and similarly for $\Delta \Lambda)$. Hence using the fact that $U(l-1) \in \mathscr{B}_{l-1}$ commutes with $\Delta A(l)$ and $\Delta \Lambda(l),(4.1)$ gives

$$
\Delta U(l) v \otimes \Phi^{\otimes k}=\left\{M^{+} \Delta A^{*}(l)+M^{\circ} \Delta t(l)\right\} U(l-1) v \otimes \Phi^{\otimes k} .
$$

Similarly, any difference equation of the form

$$
\Delta V(l)=\left\{N^{ \pm} \Delta \Lambda(l)+M^{+} \Delta A^{*}(l)+N^{-} \Delta A(l)+M^{\circ} \Delta t(l)\right\} V(l-1)
$$

satisfies

$$
\Delta V(l) v \otimes \Phi^{\otimes k}=\left\{M^{+} \Delta A^{*}(l)+M^{\circ} \Delta t(l)\right\} V(l-1) v \otimes \Phi^{\otimes k} .
$$

Hence if $V(l-1) v \otimes \Phi^{\otimes k}=U(l-1) v \otimes \Phi^{\otimes k}$, then $V(l) v \otimes \Phi^{\otimes k}=U(l) v \otimes \Phi^{\otimes k}$. By induction, $V(l) v \otimes \Phi^{\otimes k}=U(l) v \otimes \Phi^{\otimes k}$ for any $l$ if $V(0)=U(0)=I$. Thus

$$
\begin{aligned}
\mathbb{P}\left(U(l)^{*} X U(l)\right)=\langle U(l) v \otimes & \left.\Phi^{\otimes k}, X U(l) v \otimes \Phi^{\otimes k}\right\rangle \\
& =\left\langle V(l) v \otimes \Phi^{\otimes k}, X V(l) v \otimes \Phi^{\otimes k}\right\rangle=\mathbb{P}\left(V(l)^{*} X V(l)\right),
\end{aligned}
$$

regardless of what we choose for $N^{ \pm}$and $N^{-}$.

We are now free to choose $N^{ \pm}$and $N^{-}$so that $V(l)$ satisfies the remaining requirement $V(l) \in \mathscr{C}_{l}^{\prime}$. But if we choose $N^{ \pm}=0$ and $N^{-}=M^{+}$as in the statement of the lemma, it follows that $V(l) \in \mathscr{M} \otimes \mathscr{C}_{l} \subset \mathscr{C}_{l}^{\prime}$ for every $l$. Indeed, suppose that $V(l-1) \in \mathscr{M} \otimes \mathscr{C}_{l-1}$. Then $V(l)$ is defined by the recursion as a function of $V(l-1)$, $\Delta Z(l)=\Delta A(l)+\Delta A^{*}(l) \in \mathscr{C}_{l}$, and $M^{+}, M^{\circ} \in \mathscr{M}$, which is obviously contained in $\mathscr{M} \otimes \mathscr{C}_{l}$. The result then follows by induction, and the lemma is proved.

It remains for us to consider the case that $\rho$ is not a vector state. By linearity we can always write $\rho=\operatorname{Tr}[\tilde{\rho} X]$ for some $2 \times 2$ density matrix $\tilde{\rho}$. But any density matrix can be diagonalized (as it is a positive matrix), so that we can write without loss of generality $\tilde{\rho}=\lambda_{1} v_{1} v_{1}^{*}+\lambda_{2} v_{2} v_{2}^{*}$, where $v_{1}$ is the (normalized) eigenvector of $\tilde{\rho}$ with eigenvalue $\lambda_{2}$ and $v_{2}$ is the eigenvector with eigenvalue $\lambda_{2}$. As the lemma holds for each of the vector states $\rho_{1,2}(X)=\left\langle v_{1,2}, X v_{1,2}\right\rangle$, it must hold for arbitrary $\rho$.

The solution of the filtering problem is now remarkably straightforward.

Definition 6.3. For any atomic observable $X \in \mathscr{B}_{0}$, the unnormalized conditional expectation $\sigma_{l}(X) \in \mathscr{Y}_{l}$ is defined as

$$
\sigma_{l}(X)=U(l)^{*} \mathbb{P}\left(V(l)^{*} X V(l) \mid \mathscr{C}_{l}\right) U(l) .
$$

THEOREM 6.4. The unnormalized conditional expectation $\sigma_{l}(X)$ satisfies the following linear filtering equation for homodyne detection:

$$
\Delta \sigma_{l}(X)=\sigma_{l-1}(\mathcal{L}(X)) \Delta t(l)+\sigma_{l-1}(\mathcal{J}(X)) \Delta Y(l), \quad \sigma_{0}(X)=\rho(X),
$$

where $\mathcal{L}(X)$ is the discrete Lindblad generator and $\mathcal{J}(X)$ was defined in Lemma 5.8. Furthermore, the noncommutative Kallianpur-Striebel formula holds:

$$
\pi_{l}(X)=\frac{\sigma_{l}(X)}{\sigma_{l}(I)} \quad \forall X \in \mathscr{B}_{0} .
$$

Proof. The Kallianpur-Striebel formula is simply (6.1). To obtain the linear recursion, we calculate using the discrete Itô rules that

$$
\begin{aligned}
\Delta\left(V(l)^{*} X V(l)\right)=V(l-1)^{*} \mathcal{L}(X) V & (l-1) \Delta t(l) \\
& +V(l-1)^{*} \mathcal{J}(X) V(l-1)\left(\Delta A(l)+\Delta A^{*}(l)\right) .
\end{aligned}
$$

Copyright $@$ ㅇ by SIAM. Unauthorized reproduction of this article is prohibited. 
Calculating the conditional expectation with respect to $\mathscr{C}_{l}$, we obtain

$$
\begin{aligned}
& \Delta \mathbb{P}\left(V(l)^{*} X V(l) \mid \mathscr{C}_{l}\right)=\mathbb{P}\left(V(l-1)^{*} \mathcal{L}(X) V(l-1) \mid \mathscr{C}_{l}\right) \Delta t(l) \\
&+ \mathbb{P}\left(V(l-1)^{*} \mathcal{J}(X) V(l-1) \mid \mathscr{C}_{l}\right)\left(\Delta A(l)+\Delta A^{*}(l)\right)
\end{aligned}
$$

using $\Delta A(l)+\Delta A^{*}(l) \in \mathscr{C}_{l}$. But note that $\mathscr{C}_{l}=\mathscr{C}_{l-1} \otimes \operatorname{alg}\left\{\Delta A(l)+\Delta A^{*}(l)\right\}$ and that $\Delta A(l)+\Delta A^{*}(l)$ is independent of $\mathscr{M} \otimes \mathscr{C}_{l-1}$ under $\mathbb{P}$. Hence by the independence property of the conditional expectation (cf. the last property listed in Table 3.1) and the fact that $V(l-1)^{*} X V(l-1) \in \mathscr{M} \otimes \mathscr{C}_{l-1}$ for any $X \in \mathscr{B}_{0}$, we obtain

$$
\begin{aligned}
\Delta \mathbb{P}\left(V(l)^{*} X V(l) \mid \mathscr{C}_{l}\right)=\mathbb{P}( & \left.V(l-1)^{*} \mathcal{L}(X) V(l-1) \mid \mathscr{C}_{l-1}\right) \Delta t(l) \\
& +\mathbb{P}\left(V(l-1)^{*} \mathcal{J}(X) V(l-1) \mid \mathscr{C}_{l-1}\right)\left(\Delta A(l)+\Delta A^{*}(l)\right) .
\end{aligned}
$$

Now multiply from the left by $U(l)^{*}$ and from the right by $U(l)$. Note that

$$
U(l)^{*} \Delta \mathbb{P}\left(V(l)^{*} X V(l) \mid \mathscr{C}_{l}\right) U(l)=\Delta\left(U(l)^{*} \mathbb{P}\left(V(l)^{*} X V(l) \mid \mathscr{C}_{l}\right) U(l)\right),
$$

because $U(l)^{*} C U(l)=U(l-1)^{*} C U(l-1)$ for any $C \in \mathscr{C}_{l-1}$ (see section 2.5). Furthermore, $U(l)^{*}\left(\Delta A(l)+\Delta A^{*}(l)\right) U(l)=\Delta Y(l)$, and the theorem follows.

Notice how much simpler the linear recursion is compared to the nonlinear recursion obtained through the martingale method; for example, this could make digital implementation of the linear recursion more straightforward. Nonetheless the two methods should give the same answer, as they are both ultimately expressions for the same quantity $\pi_{l}(X)$. Let us verify this explicitly. Note that

$\Delta \pi_{l}(X)=\Delta\left[\frac{\sigma_{l}(X)}{\sigma_{l}(I)}\right]=\frac{\sigma_{l}(X)}{\sigma_{l}(I)}-\frac{\sigma_{l-1}(X)}{\sigma_{l-1}(I)}=\frac{\Delta \sigma_{l}(X)}{\sigma_{l-1}(I)}-\sigma_{l}(X)\left(\sigma_{l-1}(I)^{-1}-\sigma_{l}(I)^{-1}\right)$.

The first term on the right is easily evaluated as

$$
\frac{\Delta \sigma_{l}(X)}{\sigma_{l-1}(I)}=\pi_{l-1}(\mathcal{L}(X)) \Delta t(l)+\pi_{l-1}(\mathcal{J}(X)) \Delta Y(l),
$$

whereas we obtain for the second term (using $\mathcal{L}(I)=0$ )

$$
\sigma_{l}(X)\left(\sigma_{l-1}(I)^{-1}-\sigma_{l}(I)^{-1}\right)=\sigma_{l}(X) \frac{\Delta \sigma_{l}(I)}{\sigma_{l}(I) \sigma_{l-1}(I)}=\pi_{l}(X) \pi_{l-1}(\mathcal{J}(I)) \Delta Y(l) .
$$

Hence we obtain

$$
\Delta \pi_{l}(X)=\pi_{l-1}(\mathcal{L}(X)) \Delta t(l)+\left\{\pi_{l-1}(\mathcal{J}(X))-\pi_{l}(X) \pi_{l-1}(\mathcal{J}(I))\right\} \Delta Y(l) .
$$

Writing $\pi_{l}(X)=\pi_{l-1}(X)+\Delta \pi_{l}(X)$ and solving for $\Delta \pi_{l}(X)$ gives precisely the nonlinear recursion obtained in section 5.2, taking into account the identity

$$
\left(I+\pi_{l-1}(\mathcal{J}(I)) \Delta Y(l)\right)^{-1}=\frac{I-\pi_{l-1}(\mathcal{J}(I)) \Delta Y(l)}{I-\lambda^{2} \pi_{l-1}(\mathcal{J}(I))^{2}},
$$

where we have used $(\Delta Y(l))^{2}=\Delta t(l)$. This sheds some light on the seemingly complicated structure of the discrete nonlinear filtering equation.

Copyright $\odot$ by SIAM. Unauthorized reproduction of this article is prohibited. 
6.3. Photodetection. If we naively try to follow the procedure above in the photodetection case $Z(l)=\Lambda(l)$, we run into problems. Let us see what goes wrong. Following the steps in the proof of Lemma 6.2, we reach the point where we have to choose $N^{ \pm}$and $N^{-}$so that $V(l) \in \mathscr{C}_{l}^{\prime}$. But this is impossible ${ }^{2}$ because we cannot get rid of the $\Delta A^{*}$ term (as $\Delta A^{*}(l) \Phi^{\otimes k} \neq 0$ ), and $\Delta A^{*}(l)$ does not commute with $\Delta \Lambda(l)$.

We are not restricted, however, to using Lemma 6.1 with $U=U(l)$. To deal with the photodetection case, suppose that $R(l)$ is some unitary operator of the form $I \otimes R$ in $\mathscr{M} \otimes \mathscr{W}_{k}$ (i.e., it acts only on the field, not on the atom). Define the state $\mathbb{Q}^{l}(X)=\mathbb{P}\left(U(l)^{*} R(l)^{*} X R(l) U(l)\right)$ and the algebra $\mathscr{R}_{l}=R(l) \mathscr{C}_{l} R(l)^{*}$. Suppose that there is some $V(l) \in \mathscr{R}_{l}^{\prime}$ such that $\mathbb{Q}^{l}(X)=\mathbb{P}\left(V(l)^{*} X V(l)\right)$. Then by Lemma 6.1 and the Bayes formula, we have for any $X \in \mathscr{B}_{0}$ (using $R(l)^{*} X R(l)=X R(l)^{*} R(l)=X$ )

$$
\pi_{l}(X)=U(l)^{*} R(l)^{*} \mathbb{Q}^{l}\left(X \mid \mathscr{R}_{l}\right) R(l) U(l)=\frac{U(l)^{*} R(l)^{*} \mathbb{P}\left(V(l)^{*} X V(l) \mid \mathscr{R}_{l}\right) R(l) U(l)}{U(l)^{*} R(l)^{*} \mathbb{P}\left(V(l)^{*} V(l) \mid \mathscr{R}_{l}\right) R(l) U(l)} .
$$

This is precisely as before, except that we have inserted an additional rotation $R(l)$. The idea is that if we choose $R(l)$ appropriately, then $R(l)(\Delta \Lambda(l)) R(l)^{*}$ contains a $\Delta A^{*}$ term so that we can proceed as in the previous section to find a suitable $V(l) \in \mathscr{R}_{l}^{\prime}$. A possible choice of $R(l)$ is given in the following lemma.

Lemma 6.5. Define $R(l)=\exp \left(A(l)-A^{*}(l)\right), l=1, \ldots, k$. Then $R(l)$ is unitary,

$$
\Delta R(l)=\left\{\frac{\sin (\lambda)}{\lambda}\left(\Delta A(l)-\Delta A^{*}(l)\right)+\frac{\cos (\lambda)-1}{\lambda^{2}} \Delta t(l)\right\} R(l-1),
$$

and we obtain the expression

$R(l) \Delta \Lambda(l) R(l)^{*}=\left(\cos ^{2} \lambda-\sin ^{2} \lambda\right) \Delta \Lambda(l)+\frac{\sin \lambda \cos \lambda}{\lambda}\left(\Delta A(l)+\Delta A^{*}(l)\right)+\frac{\sin ^{2} \lambda}{\lambda^{2}} \Delta t(l)$.

Proof. Unitarity of $R(l)$ is immediate. To obtain the difference equation, note that $R(l)=\exp \left(\Delta A(l)-\Delta A^{*}(l)\right) R(l-1)$, so that we essentially need to calculate $\exp \left(\Delta A(l)-\Delta A^{*}(l)\right)=\left(\exp \left(\lambda \sigma_{-}-\lambda \sigma_{+}\right)\right)_{l}$. But it is not difficult to evaluate explicitly the matrix exponential of the $2 \times 2$ matrix $\lambda\left(\sigma_{-}-\sigma_{+}\right)$:

$\exp \left(\lambda\left(\sigma_{-}-\sigma_{+}\right)\right)=\exp \left[\left(\begin{array}{cc}0 & -\lambda \\ \lambda & 0\end{array}\right)\right]=\left(\begin{array}{cc}\cos \lambda & -\sin \lambda \\ \sin \lambda & \cos \lambda\end{array}\right)=\cos \lambda I+\sin \lambda\left(\sigma_{-}-\sigma_{+}\right)$.

The expression for $\Delta R(l)$ follows directly. To obtain the remaining expression, note that $R(l-1)$ commutes with $\Delta \Lambda(l)$; hence

$$
\begin{aligned}
& R(l)(\Delta \Lambda(l)) R(l)^{*}=e^{\Delta A(l)-\Delta A^{*}(l)} \Delta \Lambda(l) e^{\Delta A^{*}(l)-\Delta A(l)} \\
= & \left(\left(\begin{array}{cc}
\cos \lambda & -\sin \lambda \\
\sin \lambda & \cos \lambda
\end{array}\right)\left(\begin{array}{cc}
1 & 0 \\
0 & 0
\end{array}\right)\left(\begin{array}{cc}
\cos \lambda & \sin \lambda \\
-\sin \lambda & \cos \lambda
\end{array}\right)\right)_{l}=\left(\left(\begin{array}{cc}
\cos ^{2} \lambda & \sin \lambda \cos \lambda \\
\sin \lambda \cos \lambda & \sin ^{2} \lambda
\end{array}\right)\right)_{l},
\end{aligned}
$$

from which the result follows immediately.

\footnotetext{
${ }^{2}$ This is not surprising for the following reason. Applying the spectral theorem to the commutative algebra $\mathscr{C}_{l}$, we obtain a classical measure space $(\Omega, \mathcal{F})$ on which $\mathbb{Q}^{l}$ and $\mathbb{P}$ induce different probability measures $\mathbf{Q}$ and $\mathbf{P}$, respectively. Suppose there exists a $V \in \mathscr{C}_{l}^{\prime}$ such that $\mathbb{Q}^{l}(X)=\mathbb{P}\left(V^{*} X V\right)$. Then it is not difficult to verify that $\mathbf{Q} \ll \mathbf{P}$ with $d \mathbf{Q} / d \mathbf{P}=\iota\left(\mathbb{P}\left(V^{*} V \mid \mathscr{C}_{l}\right)\right)$. But note that $\iota(\Delta \Lambda(l))$ is distributed under $\mathbf{Q}$ in the same way as the observation increment $\Delta Y(l)$ under $\mathbb{P}$, whereas $\iota(\Delta \Lambda(l))=0 \mathbf{P}$-a.s. This contradicts $\mathbf{Q} \ll \mathbf{P}$ and hence the existence of $V$.
} 
For brevity, let us call $R(l)(\Delta \Lambda(l)) R(l)^{*}=\Delta \bar{Z}(l)$. We are now in a position to repeat Lemma 6.2 for the photodetection case.

LEмma 6.6. Let $V$ be the solution to the following difference equation:

$$
\begin{aligned}
\Delta V(l)=\{ & \left(\frac{\lambda}{\sin \lambda} M^{+}-\frac{\lambda^{2}}{\cos \lambda} M^{\circ}-\frac{1}{\cos \lambda}\right)\left(\Delta \bar{Z}(l)-\frac{\sin ^{2} \lambda}{\lambda^{2}} \Delta t(l)\right) \\
& \left.+\left(\frac{\sin \lambda}{\lambda} M^{+}+\cos \lambda M^{\circ}+\frac{\cos \lambda-1}{\lambda^{2}}\right) \Delta t(l)\right\} V(l-1), \quad V(0)=I .
\end{aligned}
$$

Then $V(l) \in \mathscr{R}_{l}^{\prime}$ and $\mathbb{P}\left(V(l)^{*} X V(l)\right)=\mathbb{P}\left(U(l)^{*} R(l)^{*} X R(l) U(l)\right) \forall X \in \mathscr{M} \otimes \mathscr{W}_{k}$.

Proof. Using the quantum Itô rules, it is not difficult to calculate

$$
\begin{aligned}
& \Delta(R(l) U(l))=\left\{(\cdots) \Delta \Lambda(l)+\left(\cos \lambda M^{+}-\lambda \sin \lambda M^{\circ}-\frac{\sin \lambda}{\lambda}\right) \Delta A^{*}(l)\right. \\
& \left.\quad+(\cdots) \Delta A(l)+\left(\frac{\sin \lambda}{\lambda} M^{+}+\cos \lambda M^{\circ}+\frac{\cos \lambda-1}{\lambda^{2}}\right) \Delta t(l)\right\} R(l-1) U(l-1) .
\end{aligned}
$$

We can now follow exactly the procedure in the proof of Lemma 6.2, and using the expression for $\Delta \bar{Z}$ obtained in Lemma 6.5 the result follows.

We can now obtain the linear filter for photodetection as before.

THEOREM 6.7. The unnormalized conditional expectation

$$
\sigma_{l}(X)=U(l)^{*} R(l)^{*} \mathbb{P}\left(V(l)^{*} X V(l) \mid \mathscr{R}_{l}\right) R(l) U(l), \quad X \in \mathscr{B}_{0},
$$

satisfies the linear filtering equation for photodetection,

$\Delta \sigma_{l}(X)=\sigma_{l-1}(\mathcal{L}(X)) \Delta t(l)+\sigma_{l-1}(\mathcal{T}(X))\left(\Delta Y(l)-\frac{\sin ^{2} \lambda}{\lambda^{2}} \Delta t(l)\right), \quad \sigma_{0}(X)=\rho(X)$,

where $\mathcal{L}(X)$ is the discrete Lindblad generator and $\mathcal{T}(X)$ is given by

$$
\mathcal{T}(X)=\frac{1}{\cos ^{2} \lambda}\left(\frac{\lambda^{2}}{\sin ^{2} \lambda} M^{+*} X M^{+}-X-\lambda^{2} \mathcal{L}(X)\right) .
$$

Furthermore, the noncommutative Kallianpur-Striebel formula holds:

$$
\pi_{l}(X)=\frac{\sigma_{l}(X)}{\sigma_{l}(I)} \quad \forall X \in \mathscr{B}_{0}
$$

Proof. We begin by calculating $\Delta\left(V(l)^{*} X V(l)\right)$ using the Itô rules; this is a tedious but straightforward calculation, and we will not repeat it here. The result is

$$
\begin{aligned}
\Delta\left(V(l)^{*} X V(l)\right)=V(l-1)^{*} \mathcal{L}(X) V(l-1) \Delta t(l) & \\
& +V(l-1)^{*} \mathcal{T}(X) V(l-1)\left(\Delta \bar{Z}(l)-\frac{\sin ^{2} \lambda}{\lambda^{2}} \Delta t(l)\right),
\end{aligned}
$$

where $\mathcal{T}(X)$ is given by the expression

$$
\mathcal{T}(X)=\frac{\lambda^{2}}{\sin ^{2} \lambda} M^{+*} X M^{+}-\frac{\lambda^{4}}{\cos ^{2} \lambda} M^{\circ *} X M^{\circ}-\frac{\lambda^{2}}{\cos ^{2} \lambda}\left(X M^{\circ}+M^{\circ *} X\right)-\frac{1}{\cos ^{2} \lambda} X .
$$

Using the expression for $\mathcal{L}(X)$, the latter is easily transformed into the form given in the theorem. The remainder of the proof is the same as that of Theorem 6.4. 
The presence of the $\sin \lambda$ and $\cos \lambda$ terms in the linear filter for photodetection is an artefact of our choice of $R(l)$. In fact, this choice is not unique: many $R(l)$ would work and give rise to different linear filters! However, the Kallianpur-Striebel formula guarantees that all these linear filters coincide when normalized with the nonlinear filter of section 5.3. To complete our discussion of linear filters, let us show explicitly how normalization of the linear filter of Theorem 6.7 gives back, through a series of miraculous cancellations, the nonlinear filter for photodetection which we obtained earlier through martingale methods. We begin by writing, as in section 6.2 ,

$$
\Delta \pi_{l}(X)=\frac{\Delta \sigma_{l}(X)}{\sigma_{l-1}(I)}-\pi_{l}(X) \frac{\Delta \sigma_{l}(I)}{\sigma_{l-1}(I)} .
$$

Using the fact that $\pi_{l}(X)=\Delta \pi_{l}(X)+\pi_{l-1}(X)$, this gives explicitly

$$
\begin{aligned}
& \Delta \pi_{l}(X)\left[I+\pi_{l-1}(\mathcal{T}(I))\left(\Delta Y(l)-\sin ^{2} \lambda\right)\right] \\
& \qquad \begin{aligned}
\lambda_{l-1}(\mathcal{L}(X)) \Delta t(l)-\frac{\sin ^{2} \lambda}{\lambda^{2}} & \left.\pi_{l-1}(\mathcal{T}(X))-\pi_{l-1}(X) \pi_{l-1}(\mathcal{T}(I))\right] \Delta t(l) \\
& +\left[\pi_{l-1}(\mathcal{T}(X))-\pi_{l-1}(X) \pi_{l-1}(\mathcal{T}(I))\right] \Delta Y(l) .
\end{aligned}
\end{aligned}
$$

Using the expression for $\mathcal{T}(X)$ and $\mathcal{L}(I)=0$, we calculate

$$
\begin{aligned}
& \pi_{l-1}(\mathcal{T}(X))-\pi_{l-1}(X) \pi_{l-1}(\mathcal{T}(I))=-\frac{\lambda^{2}}{\cos ^{2} \lambda} \pi_{l-1}(\mathcal{L}(X)) \\
& \quad+\frac{\lambda^{2}}{\sin ^{2} \lambda \cos ^{2} \lambda}\left[\pi_{l-1}\left(M^{+*} X M^{+}\right)-\pi_{l-1}(X) \pi_{l-1}\left(M^{+*} M^{+}\right)\right] .
\end{aligned}
$$

Hence we obtain

$$
\begin{aligned}
& \Delta \pi_{l}(X)\left[I+\pi_{l-1}(\mathcal{T}(I))(\Delta Y(l)-\right.\left.\left.\sin ^{2} \lambda\right)\right] \\
&=\frac{1}{\cos ^{2} \lambda}\left[\pi_{l-1}(\mathcal{L}(X))-\pi_{l-1}\left(M^{+*} X M^{+}\right)+\pi_{l-1}(X) \pi_{l-1}\left(M^{+*} M^{+}\right)\right] \Delta t(l) \\
&+\left[\pi_{l-1}(\mathcal{T}(X))-\pi_{l-1}(X) \pi_{l-1}(\mathcal{T}(I))\right] \Delta Y(l) .
\end{aligned}
$$

Next, we claim that

$$
\begin{aligned}
& {\left[I+\pi_{l-1}(\mathcal{T}(I))\left(\Delta Y(l)-\sin ^{2} \lambda\right)\right]^{-1}} \\
& \quad=\left[I+\cos ^{2} \lambda \pi_{l-1}(\mathcal{T}(I))\right]^{-1} \Delta Y(l)+\left[I-\sin ^{2} \lambda \pi_{l-1}(\mathcal{T}(I))\right]^{-1}(I-\Delta Y(l)) .
\end{aligned}
$$

The easiest way to see this is to consider $\pi_{l-1}(\mathcal{T}(I))$ and $\Delta Y(l)$ to be classical random variables through the spectral theorem; as $(\Delta Y(l))^{2}=\Delta Y(l)$ we conclude that $\Delta Y(l)$ is a $\{0,1\}$-valued random variable, and the statement follows directly. Using the explicit expression for $\mathcal{T}(X)$, we find that

$$
\left[I-\sin ^{2} \lambda \pi_{l-1}(\mathcal{T}(I))\right]^{-1}=\frac{\cos ^{2} \lambda}{I-\lambda^{2} \pi_{l-1}\left(M^{+*} M^{+}\right)}
$$

and that

$$
\left[I+\cos ^{2} \lambda \pi_{l-1}(\mathcal{T}(I))\right]^{-1}=\frac{\sin ^{2} \lambda}{\lambda^{2} \pi_{l-1}\left(M^{+*} M^{+}\right)} .
$$

Using these expressions, the remainder of the calculation is a straightforward exercise and indeed we obtain the expression for $\Delta \pi_{l}(X)$ as in section 5.3.

Copyright (C) by SIAM. Unauthorized reproduction of this article is prohibited. 
7. Feedback Control. Everything we have done up to this point has been devoid of human intervention. An atom sits in free space, emits radiation at its leisure, and all we have allowed ourselves to do is to observe the output radiation and to interpret it statistically (filtering). In this section we will allow ourselves to manipulate the atom in real time; this provides an opportunity for feedback control, which we will approach using optimal control theory (section 8) and Lyapunov methods (section 9).

In this section we carefully introduce the concepts required for feedback control. As we shall see, some subtleties arise which we explain and address using the framework developed in [17], suitably adapted to our discrete setting. Early work on quantum feedback control appears in [9, 10], and a large number of applications have been discussed (albeit not in a mathematically rigorous way) in the physics literature; see, e.g., [31] and the references therein.

In order to understand feedback, we need to consider how the atomic dynamics can be influenced by control actions, and what information is available to determine the control actions. We develop these ideas in the following two subsections. In section 7.1 we describe how a controller can influence the atomic dynamics. This will allow us to apply open loop controls, that is, we can apply a deterministic function to the control inputs of the system. Though this is not our ultimate goal, it is a helpful first step; in section 7.2 we will show how to replace this deterministic input by some function of the observation history (feedback). This is likely to be advantageous: the more information we have, the better we can control!

Remark 7.1. We take a moment at this point to discuss the usage of the term "quantum control" in the literature. Often this term is used to refer to open loop control, rather than feedback control. Such control problems can be reduced to deterministic control problems, as we will show in section 7.1. The classical analogue of this concept would be deterministic control design for the Fokker-Planck equation.

Our main goal here is to discuss quantum feedback control, where the feedback is based on an observations process obtained from the system to be controlled. This corresponds to the classical idea of a control system as consisting of a system to be controlled (the plant); a sensor which gives rise to an observations process; an actuator which allows one to modify the dynamics of the system in real time; and a controller, which is a signal processing device that takes the observations as its input and produces an actuation signal as its output.

Sometimes the term "quantum feedback control" is used in a somewhat broader context. One could consider the setup described above in the absence of the sensor component. In this case the controller must be treated as being itself a physical system, rather than a signal processing device, and the pursuit of this idea leads to rather different theory and applications (see, e.g., [85, 87, 52]). This type of feedback is usually called coherent feedback or all-optical feedback (in the context of quantum optics), to distinguish it from observation-based feedback, which we consider here.

7.I. Open Loop Control (No Feedback). To add a control input to our model, recall from section 2.5 that the time evolution of an observable $X$ is given by $j_{l}(X)=$ $U(l)^{*} X U(l)$ with repeated interaction unitary

$$
U(l)=\vec{\prod}_{i=1}^{l} M(i)=M(1) M(2) \cdots M(l), \quad U(0)=I .
$$

The interaction in every time slice was given by

$$
M(l)=e^{-i\left\{j_{l-1}\left(L_{1}\right) \Delta \Lambda(l)+j_{l-1}\left(L_{2}\right) \Delta A^{*}(l)+j_{l-1}\left(L_{2}^{*}\right) \Delta A(l)+j_{l-1}\left(L_{3}\right) \Delta t(l)\right\}},
$$


where $L_{1}, L_{2}, L_{3} \in \mathscr{B}_{0}$ are atomic operators. To add a control input, we simply allow ourselves a choice of different $M(l)$ 's in every time step. To be precise, let us introduce a control set $\mathfrak{U}$ (i.e., these are the values the control can take), and for each $u \in \mathfrak{U}$ we define a set of atomic operators $L_{1}(u), L_{2}(u), L_{3}(u) \in \mathscr{B}_{0}$. An open loop control strategy is a $k$-tuple $\mathbf{u}=\left\{u_{1}, \ldots, u_{k}\right\}$, where $u_{i} \in \mathfrak{U}$ for all $i$, and the corresponding time evolution $j_{l}^{\mathbf{u}}(X)=U(l, \mathbf{u})^{*} X U(l, \mathbf{u})$ is defined by

$$
U(l, \mathbf{u})=\vec{\prod}_{i=1}^{l} M^{\mathbf{u}}\left(i, u_{i}\right)=M^{\mathbf{u}}\left(1, u_{1}\right) M^{\mathbf{u}}\left(2, u_{2}\right) \cdots M^{\mathbf{u}}\left(l, u_{l}\right), \quad U(0, \mathbf{u})=I,
$$

where the single time slice interaction unitary is given by

$$
M^{\mathbf{u}}(l, u)=e^{-i\left\{j_{l-1}^{\mathbf{u}}\left(L_{1}(u)\right) \Delta \Lambda(l)+j_{l-1}^{\mathbf{u}}\left(L_{2}(u)\right) \Delta A^{*}(l)+j_{l-1}^{\mathbf{u}}\left(L_{2}(u)^{*}\right) \Delta A(l)+j_{l-1}^{\mathbf{u}}\left(L_{3}(u)\right) \Delta t(l)\right\}} .
$$

Note that $M^{\mathbf{u}}(l, u)$, for fixed $u$, depends only on $\mathbf{u}$ through $u_{1}, \ldots, u_{l-1}$, and similarly $U(l, \mathbf{u})$ depends only on $u_{1}, \ldots, u_{l}$. We write $U(l, \mathbf{u})$ rather than $U\left(l, u_{1}, \ldots, u_{l}\right)$ purely for notational convenience; the latter would technically be more appropriate!

As before, it is convenient to run the definition backwards in time, i.e.,

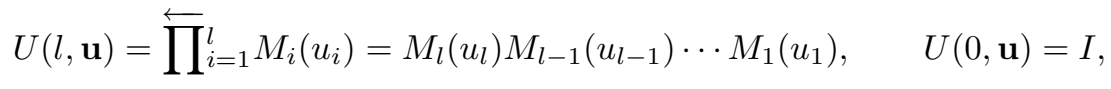

where $M_{l}(u)$ is given by

$$
M_{l}(u)=e^{-i\left\{L_{1}(u) \Delta \Lambda(l)+L_{2}(u) \Delta A^{*}(l)+L_{2}(u)^{*} \Delta A(l)+L_{3}(u) \Delta t(l)\right\}} .
$$

These operators are functions of the current control value and do not depend on the full sequence $\mathbf{u}$. The corresponding difference equation is written as

$$
\begin{aligned}
\Delta U(l, \mathbf{u})=\{ & M^{ \pm}\left(u_{l}\right) \Delta \Lambda(l)+M^{+}\left(u_{l}\right) \Delta A^{*}(l) \\
& \left.+M^{-}\left(u_{l}\right) \Delta A(l)+M^{\circ}\left(u_{l}\right) \Delta t(l)\right\} U(l-1, \mathbf{u}), \quad U(0, \mathbf{u})=I .
\end{aligned}
$$

One could imagine the different controls $u \in \mathfrak{U}$ to correspond to different values of a magnetic field which is applied to the atom and can be changed by the experimenter in each time step. Another common control input is obtained using a laser whose amplitude can be controlled by the experimenter. We will discuss a specific example in the context of the discrete model in section 8.3.

The question of controller design could already be posed at this deterministic level. Though this rules out feedback control (as the observations are a random process), open loop controls for quantum systems have already generated important applications. For example, optimal control theory has allowed the design of timeoptimal pulse sequences for nuclear magnetic resonance (NMR) spectroscopy that significantly outperform the state of the art for that technology [58].

Open loop control design is beyond the scope of this article, so we will not go into detail. Let us take a moment, however, to make a connection with this literature. Let $X \in \mathscr{B}_{0}$ be some atomic operator; then using the quantum Itô rules, we easily establish as in section 4.2 that the expectation of the controlled time evolution $j_{l}^{\mathbf{u}}(X)$ in the case of deterministic $\mathbf{u}$ satisfies

$$
\frac{\Delta \mathbb{P}\left(j_{l}^{\mathbf{u}}(X)\right)}{\Delta t}=\mathbb{P}\left(j_{l-1}^{\mathbf{u}}\left(\mathcal{L}\left(X, u_{l}\right)\right)\right)
$$

where the controlled Lindblad generator is given by

$$
\mathcal{L}(X, u)=M^{+}(u)^{*} X M^{+}(u)+\lambda^{2} M^{\circ}(u)^{*} X M^{\circ}(u)+M^{\circ}(u)^{*} X+X M^{\circ}(u) .
$$


As $\mathbb{P}\left(j_{l}^{\mathbf{u}}(X)\right)$ is linear in $X$, we can introduce a (deterministic) $2 \times 2$ matrix $\nu_{l}^{\mathbf{u}}$ such that $\mathbb{P}\left(j_{l}^{\mathbf{u}}(X)\right)=\operatorname{Tr}\left[\nu_{l}^{\mathbf{u}} X\right]$; then (7.3) can be written as a deterministic recursion for $\nu_{l}^{\mathrm{u}}$, known as the (controlled, discrete) master equation:

$\frac{\Delta \nu_{l}^{\mathbf{u}}}{\Delta t}=M^{+}\left(u_{l}\right) \nu_{l-1}^{\mathbf{u}} M^{+}\left(u_{l}\right)^{*}+\lambda^{2} M^{\circ}\left(u_{l}\right) \nu_{l-1}^{\mathbf{u}} M^{\circ}\left(u_{l}\right)^{*}+M^{\circ}\left(u_{l}\right) \nu_{l-1}^{\mathbf{u}}+\nu_{l-1}^{\mathbf{u}} M^{\circ}\left(u_{l}\right)^{*}$.

The control goal in such a scenario is generally formulated as the desire to choose $\mathbf{u}$ so that the expectation of a certain atomic observable, or a nonlinear function of such expectations, is maximized. But these expectations can be obtained in closed form by solving the master equation, so that the control problem reduces to a deterministic optimal control problem for the master equation. This is precisely the sort of problem that is solved in [58] (in a continuous time context).

7.2. Closed Loop Control (Feedback). We now turn to the issue of letting the control at time step $l$ be a function of the observation history prior to time $l$. Mathematically, it is not entirely clear how to modify the description in the previous section to allow for feedback in the repeated interaction model; there are in fact some subtleties. It is the goal of this section to clear up this point. For notational simplicity we assume from this point onwards that $\mathfrak{U} \subset \mathbb{R}$, i.e., that our control input is a real scalar (this is not essential; everything we will do can be generalized).

Let us first consider what we mean by a controller. A controller is a signal processing device - a black box - that on every time step takes an observation $\Delta y$ as its input and generates a control signal in $\mathfrak{U}$ as its output. The most general control strategy $\mu$ is thus described by a set of functions, one for each time step:

$$
\mu=\left\{f_{1}, f_{2}\left(\Delta y_{1}\right), f_{3}\left(\Delta y_{1}, \Delta y_{2}\right), \ldots, f_{k}\left(\Delta y_{1}, \ldots, \Delta y_{k-1}\right)\right\} .
$$

Causality is enforced explicitly by making the control in time step $l$ a function only of the observations up to that time step. The feedback control $\mu$ is called admissible if $f_{l}$ takes values in $\mathfrak{U}$ for every $l$. We call the set of all admissible controls $\mathfrak{K}$.

The functions $f_{l}$ encode the input-output behavior of the controller implementing the admissible strategy $\mu \in \mathfrak{K}$. We now need to hook up the controller to our model of the system. As the observation at time $i$ is described by the observable $\Delta Y_{i}$, we define the output of the controller at time $l$ as the observable

$$
\mathfrak{u}_{l}=f_{l}(\Delta Y(1), \ldots, \Delta Y(l-1)) \in \mathscr{Y}_{l-1} .
$$

Clearly $\iota\left(\mathfrak{u}_{l}\right)$ is a random variable that takes values in $\mathfrak{U}$, precisely as it should be.

It now remains to close the loop, i.e., to make the time evolution a function of the output $\mathfrak{u}_{l}$ of the controller. Formally, we can proceed exactly as in the open loop case; i.e., we define the time evolution $j_{l}^{\mu}(X)=U^{\mu}(l)^{*} X U^{\mu}(l)$ by

$$
U^{\mu}(l)=\vec{\prod}_{i=1}^{l} M^{\mu}\left(i, \mathfrak{u}_{i}\right)=M^{\mu}\left(1, \mathfrak{u}_{1}\right) M^{\mu}\left(2, \mathfrak{u}_{2}\right) \cdots M^{\mu}\left(l, \mathfrak{u}_{l}\right), \quad U^{\mu}(0)=I,
$$

where the single time slice interaction unitary is given by

$$
M^{\mu}(l, u)=j_{l-1}^{\mu}\left(M_{l}(u)\right)
$$

To make this precise, however, we need to define what we mean by composing the unitary operator-valued function $M^{\mu}(l, u)$ with the observable $\mathfrak{u}_{l}$. We will thus take a moment to talk about such compositions. 
Composition of an Operator-Valued Function and an Observable. Let us begin with a simple classical analogy. Let $\mathfrak{u}$ be a random variable in $\ell^{\infty}(\Omega, \mathcal{F}, \mathbf{P})$, where $\Omega$ is a finite set. Then $\mathfrak{u}$ takes a finite number of values, and we will suppose these lie in a set $\mathfrak{U}$. Let $M: \mathfrak{U} \rightarrow V$ be a map from $\mathfrak{U}$ to some linear space $V$. We would like to define $M(\mathfrak{u})$ as a $V$-valued random variable. We could do this as follows:

$$
M(\mathfrak{u})(\omega)=\sum_{u \in \operatorname{ran} \mathfrak{u}} M(u) \chi_{\{\mathfrak{u}=u\}}(\omega),
$$

where ran $\mathfrak{u}$ is the range of $\mathfrak{u}$ and $\chi_{\{\mathfrak{u}=u\}}$ is the indicator function on $\{\omega: \mathfrak{u}(\omega)=u\}$. This is particularly convenient if we want to think of $\ell^{\infty}(\Omega, \mathcal{F}, \mathbf{P})$ as being itself a linear space; by elementary linear algebra the set of $V$-valued random variables is isomorphic to $V \otimes \ell^{\infty}(\Omega, \mathcal{F}, \mathbf{P})$, and the definition of $M(\mathfrak{u})$ above only involves sums and tensor products $\left(M(u) \otimes \chi_{\{\mathfrak{u}=u\}}\right)$, which are naturally defined in this space.

Though this is obviously not the simplest way of defining composition in the classical case, (7.4) looks the most natural in the noncommutative context. Let us consider the algebra $\mathscr{A} \otimes \mathscr{C}$, where $\mathscr{C}$ is commutative but $\mathscr{A}$ not necessarily so. We can think of $\mathscr{A} \otimes \mathscr{C}$ as a linear space of $\mathscr{A}$-valued random variables; indeed, applying the spectral theorem to $\mathscr{C}$ we find that $\mathscr{A} \otimes \mathscr{C} \simeq \mathscr{A} \otimes \ell^{\infty}(\mathcal{F}) \simeq \ell^{\infty}(\mathcal{F} ; \mathscr{A})$. Now suppose we are given a map $M: \mathfrak{U} \rightarrow \mathscr{A}$ and an observable $\mathfrak{u} \in \mathscr{C}$ such that $\iota(\mathfrak{u})$ takes values in $\mathfrak{U}$. Then it is natural to define the composition $M(\mathfrak{u})$ as an element in $\mathscr{A} \otimes \mathscr{C}$ in the same way as (7.4). This motivates the following definition.

Definition 7.2. Let $\mathscr{A}, \mathscr{C}$ be ${ }^{*}$-algebras where $\mathscr{C}$ is commutative. Let $\mathfrak{U} \subset \mathbb{R}$, $M: \mathfrak{U} \rightarrow \mathscr{A}$, and let $\mathfrak{u} \in \mathscr{C}$ be such that $\operatorname{sp} \mathfrak{u}=\operatorname{ran} \iota(\mathfrak{u})$, the spectrum of $\mathfrak{u}$, is a subset of $\mathfrak{U}$. Then the composition $M(\mathfrak{u}) \in \mathscr{A} \otimes \mathscr{C}$ is defined by

$$
M(\mathfrak{u})=\sum_{u \in \operatorname{sp}(\mathfrak{u})} M(u) P_{\mathfrak{u}}(u)
$$

where $P_{\mathfrak{u}}(u)=\iota^{-1}\left(\chi_{\{\iota(\mathfrak{u})=u\}}\right)$ is the eigenspace projector of $\mathfrak{u}$ for eigenvalue $u$.

In what follows it will be important to understand how the composition $M(\mathfrak{u})$ behaves under unitary transformations. Consider $\mathscr{A} \otimes \mathscr{C} \subset \mathscr{B}$, and let $U$ be a unitary operator in $\mathscr{B}$. Consider the map $M_{U}: \mathfrak{U} \rightarrow U^{*} \mathscr{A} U$ defined by $M_{U}(u)=U^{*} M(u) U$ for all $u \in \mathfrak{U}$. Then $U^{*} M(\mathfrak{u}) U \in U^{*}(\mathscr{A} \otimes \mathscr{C}) U=U^{*} \mathscr{A} U \otimes U^{*} \mathscr{C} U$ is given by

$U^{*} M(\mathfrak{u}) U=\sum_{u \in \operatorname{sp}(\mathfrak{u})} U^{*} M(u) U U^{*} P_{\mathfrak{u}}(u) U=\sum_{u \in \operatorname{sp}(\mathfrak{u})} U^{*} M(u) U P_{U^{*} \mathfrak{u} U}(u)=M_{U}\left(U^{*} \mathfrak{u} U\right)$.

Hence unitary rotations preserve the compositions defined above, as long as we remember to rotate both the observable $\mathfrak{u}$ and the map $M(u)$.

Controlled Quantum Flows. Let us return to the controlled time evolution $U^{\mu}(l)$. Note that $M^{\mu}(l, \cdot): \mathfrak{U} \rightarrow j_{l-1}^{\mu}\left(\mathscr{M} \otimes \mathscr{M}_{l}\right)$, whereas $\mathfrak{u}_{l} \in \mathscr{Y}_{l-1}=j_{l-1}^{\mu}\left(\mathscr{C}_{l-1}\right)$. Hence according to Definition 7.2, the composition $M\left(\mathfrak{u}_{l}\right)$ makes sense as an operator in the algebra $j_{l-1}^{\mu}\left(\mathscr{M} \otimes \mathscr{C}_{l-1} \otimes \mathscr{M}\right) \subset \mathscr{B}_{l}$, and by $(7.5)$

$$
M\left(l, \mathfrak{u}_{l}\right)=M(l, f(\Delta Y(1), \ldots, \Delta Y(l-1)))=j_{l-1}^{\mu}\left(M_{l}(f(\Delta Z(1), \ldots, \Delta Z(l-1)))\right) .
$$

For brevity, we will write

$$
\check{\mathfrak{u}}_{l}=f_{l}(\Delta Z(1), \ldots, \Delta Z(l-1)) \in \mathscr{C}_{l-1}
$$


so that $M\left(l, \mathfrak{u}_{l}\right)=j_{l-1}^{\mu}\left(M_{l}\left(\check{\mathfrak{u}}_{l}\right)\right)$. In particular, we can now express $U^{\mu}(l)$ as

$$
U^{\mu}(l)=\overleftarrow{\prod}_{i=1}^{l} M_{i}\left(\check{\mathfrak{u}}_{i}\right)=M_{l}\left(\check{\mathfrak{u}}_{l}\right) M_{l-1}\left(\check{\mathfrak{u}}_{l-1}\right) \cdots M_{1}\left(\check{\mathfrak{u}}_{1}\right), \quad U^{\mu}(0)=I
$$

which gives the controlled quantum stochastic difference equation

$$
\begin{aligned}
\Delta U^{\mu}(l)=\left\{M^{ \pm}\left(\check{\mathfrak{u}}_{l}\right) \Delta \Lambda(l)+M^{+}\left(\check{\mathfrak{u}}_{l}\right) \Delta A^{*}(l)\right. \\
\left.\quad+M^{-}\left(\check{\mathfrak{u}}_{l}\right) \Delta A(l)+M^{\circ}\left(\check{\mathfrak{u}}_{l}\right) \Delta t(l)\right\} U^{\mu}(l-1), \quad U^{\mu}(0)=I .
\end{aligned}
$$

The main thing to note is that in order to close the loop in (7.2), the open loop controls $u_{l}$ should be replaced by $\check{\mathfrak{u}}_{l} \in \mathscr{C}_{l-1}$ rather than $\mathfrak{u}_{l} \in \mathscr{Y}_{l-1}$. This ensures that the corresponding flow $j_{l}^{\mu}(X)$ depends on the observations history in the right way, by virtue of (7.5). Other than this subtlety, the treatment of closed loop time evolution proceeds along much the same lines as in the absence of feedback.

The notion of a controlled quantum flow [17] summarizes these ideas in a general context. The following definition is a discrete version of this concept and defines a generic repeated interaction model with scalar feedback.

DEFINITION 7.3 (controlled quantum flow). The quadruple $(\mathfrak{U}, M, Z, \mu)$ s.t.

1. $\mathfrak{U} \subset \mathbb{R}$;

2. $M_{l}: \mathfrak{U} \rightarrow \mathscr{M} \otimes \mathscr{M}_{l}, M_{l}(u)$ is a unitary operator of the form (7.1) $\forall u \in \mathfrak{U}$;

3. $Z$ is an adapted process $Z(l) \in \mathscr{W}_{l}, l=1, \ldots, k$, such that $Z(l)$ is self-adjoint and $\mathscr{C}_{l}=\operatorname{alg}\{Z(i): i=1, \ldots, l\}$ is commutative for every $l$;

4. $\mu \in \mathfrak{K}$ is an admissible control strategy,

defines a controlled quantum flow $j_{l}^{\mu}(X)=U^{\mu}(l)^{*} X U^{\mu}(l)$. Here $U^{\mu}(l)$ is given by (7.6) and the corresponding observations process $Y^{\mu}(l)$ is given by

$$
\Delta Y^{\mu}(l)=U^{\mu}(l)^{*} \Delta Z(l) U^{\mu}(l), \quad l=1, \ldots, k .
$$

Remark 7.4. For illustrative purposes, we will concentrate in what follows on the homodyne detection case $Z=A+A^{*}$; the theory for photodetection $Z=\Lambda$ proceeds along the same lines. The notion of a controlled quantum flow is much more general, however, and even allows for feedback to the detection apparatus. For example, recall that a homodyne detection setup can measure any of the processes $e^{i \varphi} A+e^{-i \varphi} A^{*}$. We could now make $\varphi$ a function of the past observations (i.e., $\left.\varphi(l)=\tilde{f}_{l}(\Delta Z(1), \ldots, \Delta Z(l-1))\right)$, thus feeding back to the homodyne detector; this fits within the framework of the controlled quantum flow as it just requires us to use a "nonlinear" $Z$. Feedback to the detector has proven to be useful for sensitive measurements of the phase of an optical pulse; see [84,3]. We will not consider this further here, but everything we will discuss can be adapted to this case as well. We encourage readers to work out the following sections for the feedback scenarios of their choice!

7.3. Filtering in the Presence of Feedback. Now that we have resolved how to model a quantum system with feedback, the next question to be resolved is whether filtering still works in this context. Fortunately this is indeed the case, and in fact little changes in the proofs. The resulting filters are completely intuitive: one obtains the same filter from a controlled quantum flow as one would obtain by first calculating the filter with an open loop control, then substituting the feedback law into the filtering equation. In this section we will briefly discuss filtering in the presence of feedback using the reference probability method. From this point onwards we restrict ourselves to the homodyne detection case $Z=A+A^{*}$. 
Fix an admissible feedback control strategy $\mu \in \mathfrak{K}$ and the corresponding control observables $\mathfrak{u}_{l}, \check{\mathfrak{u}}_{l}$. We wish to find an expression for $\pi_{l}^{\mu}(X)=\mathbb{P}\left(j_{l}^{\mu}(X) \mid \mathscr{Y}_{l}^{\mu}\right)$. For this to make sense we have to make sure that the self-nondemolition and nondemolition properties still hold. If they do not, then we did something wrong (recall that these properties are essential for a meaningful interpretation of the theory), but let us take a moment to verify that everything is as it should be.

LEMma 7.5. The observation algebra $\mathscr{Y}_{l}^{\mu}$ is commutative (self-nondemolition) and $j_{l}^{\mu}(X) \in\left(\mathscr{Y}_{l}^{\mu}\right)^{\prime}$ (nondemolition) for every $l=1, \ldots, k$ and $X \in \mathscr{M} \otimes \mathscr{C}_{l}$.

Proof. The unitary $M_{l}\left(\breve{\mathfrak{u}}_{l}\right)$, by construction, commutes with every element of $\mathscr{C}_{l-1}$. Hence $U^{\mu}(l)^{*} \Delta Z(i) U^{\mu}(l)=\Delta Y^{\mu}(i)$ for every $i \leq l-1$, and we find that

$$
\left[\Delta Y^{\mu}(i), \Delta Y^{\mu}(l)\right]=U^{\mu}(l)^{*}[\Delta Z(i), \Delta Z(l)] U^{\mu}(l)=0 .
$$

This establishes self-nondemolition. Nondemolition is established similarly.

To apply the reference probability method, we need a suitable change of state. The proof of the following lemma is omitted as it is identical to that of Lemma 6.2.

LEMma 7.6. Let $V^{\mu}$ be the solution of the following difference equation:

$$
\Delta V^{\mu}(l)=\left\{M^{+}\left(\check{\mathfrak{u}}_{l}\right)\left(\Delta A(l)+\Delta A^{*}(l)\right)+M^{\circ}\left(\check{\mathfrak{u}}_{l}\right) \Delta t(l)\right\} V^{\mu}(l-1), \quad V^{\mu}(0)=I .
$$

Then $V^{\mu}(l) \in \mathscr{C}_{l}^{\prime}$ and $\mathbb{P}\left(V^{\mu}(l)^{*} X V^{\mu}(l)\right)=\mathbb{P}\left(U^{\mu}(l)^{*} X U^{\mu}(l)\right)$ for any $X \in \mathscr{M} \otimes \mathscr{W}_{k}$.

From Lemma 3.4, we immediately obtain the Kallianpur-Striebel formula,

$$
\pi_{l}^{\mu}(X)=\frac{\sigma_{l}^{\mu}(X)}{\sigma_{l}^{\mu}(I)} \quad \forall X \in \mathscr{M} \otimes \mathscr{C}_{l}, \quad \sigma_{l}^{\mu}(X)=U^{\mu}(l)^{*} \mathbb{P}\left(V^{\mu}(l)^{*} X V^{\mu}(l) \mid \mathscr{C}_{l}\right) U^{\mu}(l),
$$

and following the proof of Theorem 6.4 gives the following unnormalized controlled filtering equation for homodyne detection:

$$
\Delta \sigma_{l}^{\mu}(X)=\sigma_{l-1}^{\mu}\left(\mathcal{L}\left(X, \check{\mathfrak{u}}_{l}\right)\right) \Delta t(l)+\sigma_{l-1}^{\mu}\left(\mathcal{J}\left(X, \check{\mathfrak{u}}_{l}\right)\right) \Delta Y^{\mu}(l), \quad \sigma_{0}^{\mu}(X)=\rho(X) .
$$

Here we have used the controlled Lindblad generator

$$
\mathcal{L}(X, u)=M^{+}(u)^{*} X M^{+}(u)+\lambda^{2} M^{\circ}(u)^{*} X M^{\circ}(u)+M^{\circ}(u)^{*} X+X M^{\circ}(u),
$$

and we have written

$$
\mathcal{J}(X, u)=X M^{+}(u)+M^{+}(u)^{*} X+\lambda^{2} M^{\circ}(u)^{*} X M^{+}(u)+\lambda^{2} M^{+}(u)^{*} X M^{\circ}(u) .
$$

As in section 6.2, we can also normalize this equation; this gives rise to the following nonlinear controlled filtering equation for homodyne detection:

$$
\begin{aligned}
\Delta \pi_{l}^{\mu}(X)= & \pi_{l-1}^{\mu}\left(\mathcal{L}\left(X, \check{\mathfrak{u}}_{l}\right)\right) \Delta t(l) \\
& +\frac{\pi_{l-1}^{\mu}\left(\mathcal{J}\left(X, \check{\mathfrak{u}}_{l}\right)\right)-\pi_{l-1}^{\mu}\left(X+\lambda^{2} \mathcal{L}\left(X, \check{\mathfrak{u}}_{l}\right)\right) \pi_{l-1}^{\mu}\left(\mathcal{J}\left(I, \check{\mathfrak{u}}_{l}\right)\right)}{I-\lambda^{2} \pi_{l-1}^{\mu}\left(\mathcal{J}\left(I, \check{\mathfrak{u}}_{l}\right)\right)^{2}} \\
& \times\left(\Delta Y^{\mu}(l)-\pi_{l-1}^{\mu}\left(\mathcal{J}\left(I, \check{\mathfrak{u}}_{l}\right)\right) \Delta t(l)\right) .
\end{aligned}
$$

Finally, we claim that even in the controlled case $\Delta Y^{\mu}(l)-\pi_{l-1}^{\mu}\left(\mathcal{J}\left(I, \check{\mathfrak{u}}_{l}\right)\right) \Delta t(l)$ is a martingale. To show this, it suffices to demonstrate that $\pi_{l-1}^{\mu}\left(\mathcal{J}\left(I, \breve{\mathfrak{u}}_{l}\right)\right) \Delta t(l)$ is the predictable part in the Doob decomposition of $\Delta Y^{\mu}(l)$, but this follows exactly as in the proof of the uncontrolled counterpart of this result, Lemma 5.7.

Copyright (c) by SIAM. Unauthorized reproduction of this article is prohibited. 
7.4. The Controlled Quantum Filter. Since $\pi_{l}^{\mu}(X)$ is linear in $X$, we can find a random $2 \times 2$ matrix $\rho_{l}^{\mu}$ such that $\iota\left(\pi_{l}^{\mu}(X)\right)=\operatorname{Tr}\left[\rho_{l}^{\mu} X\right]$ for every $X \in \mathscr{M}$. In fact, the conditional density matrix $\rho_{l}^{\mu}$ satisfies the recursion

$$
\rho_{l}^{\mu}=\Gamma\left(\rho_{l-1}^{\mu}, u_{l}^{\mu}, \Delta y_{l}^{\mu}\right),
$$

where $y_{l}^{\mu}=\iota\left(Y^{\mu}(l)\right), u_{l}^{\mu}=\iota\left(\mathfrak{u}_{l}\right)=f_{l}\left(\Delta y_{1}^{\mu}, \ldots, \Delta y_{l-1}^{\mu}\right)$,

$$
\begin{aligned}
& \Gamma(\rho, u, \Delta y)=\rho+\overline{\mathcal{L}}(\rho, u) \lambda^{2}+\frac{\overline{\mathcal{J}}(\rho, u)-\operatorname{Tr}[\overline{\mathcal{J}}(\rho, u)]\left(\rho+\lambda^{2} \overline{\mathcal{L}}(\rho, u)\right)}{1-\lambda^{2} \operatorname{Tr}[\overline{\mathcal{J}}(\rho, u)]^{2}} \\
& \times\left(\Delta y-\operatorname{Tr}[\overline{\mathcal{J}}(\rho, u)] \lambda^{2}\right),
\end{aligned}
$$

and

$$
\begin{aligned}
& \overline{\mathcal{L}}(\rho, u)=M^{+}(u) \rho M^{+}(u)^{*}+\lambda^{2} M^{\circ}(u) \rho M^{\circ}(u)^{*}+M^{\circ}(u) \rho+\rho M^{\circ}(u)^{*}, \\
& \overline{\mathcal{J}}(\rho, u)=M^{+}(u) \rho+\rho M^{+}(u)^{*}+\lambda^{2} M^{+}(u) \rho M^{\circ}(u)^{*}+\lambda^{2} M^{\circ}(u) \rho M^{+}(u)^{*} .
\end{aligned}
$$

To obtain the recursion (7.9) we can essentially follow the procedure used in section 5.4 for the uncontrolled case. The only subtlety here is that we need to deal with the presence of feedback in terms such as $\pi_{l-1}^{\mu}\left(X M^{+}\left(\check{\mathfrak{u}}_{l}\right)\right)$ that occur in the recursion for $\pi_{l}^{\mu}(X)$. The following lemma shows how to do this; it is comparable to the classical statement $\mathbf{E}[f(X, Y) \mid Y=y]=\mathbf{E}[f(X, y) \mid Y=y]$.

Lemma 7.7. Consider a map $X: \mathfrak{U} \rightarrow \mathscr{M}$ and an observable $\check{\mathfrak{u}} \in \mathscr{C}_{l}$ such that $u^{\mu}=\iota\left(U^{\mu}(l)^{*} \check{\mathfrak{u}} U^{\mu}(l)\right)$ takes values in $\mathfrak{U}$. Then $\left.\iota\left(\pi_{l}^{\mu}[X(\check{\mathfrak{u}})]\right)=\operatorname{Tr}\left[\rho_{l} X\left(u^{\mu}\right)\right)\right]$.

Proof. Using Definition 7.2, we have

$$
\begin{aligned}
\pi_{l}[X(\check{\mathfrak{u}})] & =\pi_{l}\left[\sum_{u \in \operatorname{sp}(\check{\mathfrak{u}})} X(u) P_{\mathfrak{u}}(u)\right] \\
& =\sum_{u \in \operatorname{sp}(\mathfrak{\mathfrak { u }})} \mathbb{P}\left[U^{\mu}(l)^{*} X(u) P_{\mathfrak{u}}(u) U^{\mu}(l) \mid \mathscr{Y} l\right] \\
& =\sum_{u \in \operatorname{sp}(\mathfrak{\mathfrak { u }})} \mathbb{P}\left[U^{\mu}(l)^{*} X(u) U^{\mu}(l) \mid \mathscr{Y} l\right] U^{\mu}(l)^{*} P_{\mathfrak{u}}(u) U^{\mu}(l) \\
& =\sum_{u \in \operatorname{sp}(\mathfrak{\mathfrak { u }})} \pi_{l}[X(u)] P_{U^{\mu}(l)^{*} \mathfrak{u} U^{\mu}(l)^{*}}(u) .
\end{aligned}
$$

Applying $\iota$ to both sides, the result follows immediately.

It is sometimes more convenient to use the unnormalized form of the filter. As $\sigma_{l}^{\mu}(X)$ is linear in $X$, we can proceed exactly as before to find a random $2 \times 2$ matrix $\varrho_{l}^{\mu}$ such that $\iota\left(\sigma_{l}^{\mu}(X)\right)=\operatorname{Tr}\left[\varrho_{l}^{\mu} X\right]$ for every $X \in \mathscr{M}$. The result of Lemma 7.7 is easily shown to hold also for $\sigma_{l}^{\mu}(\cdot)$, and we obtain

$$
\varrho_{l}^{\mu}=\Sigma\left(\varrho_{l-1}^{\mu}, u_{l}^{\mu}, \Delta y_{l}^{\mu}\right),
$$

where

$$
\Sigma(\varrho, u, \Delta y)=\rho+\overline{\mathcal{L}}(\varrho, u) \lambda^{2}+\overline{\mathcal{J}}(\nu, u) \Delta y
$$

The conditional density matrix $\rho_{l}^{\mu}$ can then be calculated as $\rho_{l}^{\mu}=\varrho_{l}^{\mu} / \operatorname{Tr}\left[\varrho_{l}^{\mu}\right]$.

Copyright (c) by SIAM. Unauthorized reproduction of this article is prohibited. 
Remark 7.8. The filters (7.9) and (7.10) define classical controlled Markov processes [60, Chapter 4], where future increments depend explicitly on previous control actions and on a commutative driving process $\Delta y_{l}^{\mu}$. By analogy with the classical case, one could even consider the controlled quantum flow to be a kind of "quantum controlled Markov process," though a precise statement of this concept is not yet defined in the literature (but see also Remark 4.5).

7.5. Separated Strategies. Recall that an admissible strategy $\mu \in \mathfrak{K}$ is defined by a set of feedback functions $\mu=\left\{f_{1}, \ldots, f_{k}\right\}$ that describe how the control values depend on the measurement record. Any causal control strategy can be written in this form. We remarked in section 3.4, however, that in many cases the controls need not have an arbitrarily complicated dependence on the measurement record - it is sufficient to make the feedback at every time step a function of the filter only. In other words, many control goals can be attained using only feedback of the form

$$
u_{l}^{\mu}=g_{l-1}\left(\rho_{l-1}^{\mu}\right), \quad l=1, \ldots, k,
$$

where $g_{l}$ takes values in $\mathfrak{U}$ for any $l$. Note that such a strategy is in fact admissible as $\rho_{l-1}^{\mu}$ is a function of $\Delta y_{1}^{\mu}, \ldots, \Delta y_{l-1}^{\mu}$ only, so that we could always write $g_{l-1}\left(\rho_{l-1}^{\mu}\right)=$ $f_{l}\left(\Delta y_{1}^{\mu}, \ldots, \Delta y_{l-1}^{\mu}\right)$ for some function $f_{l}$.

Both in the case of optimal control (section 8) and in control design using Lyapunov functions (section 9), we will see that the theory leads very naturally to strategies of the form (7.12). This is highly desirable; not only does such structure significantly simplify the control design procedure, but the resulting controls are also much easier to implement in practice. Note that to implement an arbitrary admissible strategy $\mu \in \mathfrak{K}$, the controller must have enough internal memory to store the entire observation history. One has no other choice, as the feedback signal may be an arbitrary function of the control history. On the other hand, to implement a strategy of the form (7.12) the controller only needs to store the current density matrix $\rho_{l}^{\mu}$ at any time. The density matrix is then updated recursively using the filter (7.9).

Control strategies of the form (7.12) are called separated control strategies, as the controller separates into two parts: a filtering step, which can be solved recursively as described above, and a control step, which reduces to the simple evaluation of some deterministic function of the filter state. The structure of the separated controller is illustrated in Figure 7.1. The ubiquity of this separation structure highlights the fundamental importance of filtering in (quantum) stochastic control.

Let us finally fix the notation. An admissible separated strategy $\mu$ is given by

$$
\mu=\left\{g_{0}(\rho), g_{1}(\rho), \ldots, g_{k-1}(\rho)\right\},
$$

where $g_{i}: \mathcal{S} \rightarrow \mathfrak{U}$ are functions on the set $\mathcal{S}$ of $2 \times 2$ density matrices (self-adjoint, nonnegative, unit trace $2 \times 2$ matrices). We denote the set of all admissible separated strategies by $\mathfrak{K}_{S}$, and the corresponding feedback signal is given by (7.12).

Unlike in the absence of control (see section 5.5), the controlled filter $\rho_{l}^{\mu}$ need not be a Markov process as in general $\mu \in \mathfrak{K}$ may have an arbitrary dependence on the observation history ( $\rho_{l}^{\mu}$ is a controlled Markov process in the sense of [60, Chapter 4]). On the other hand, for separated controls $\mu \in \mathfrak{K}_{S}$ the process $\rho_{t}^{\mu}$ is in fact Markov. The following lemma can be proved as in section 5.5.

Lemma 7.9. For separated $\mu \in \mathfrak{K}_{S}$, the filter $\rho_{l}^{\mu}$ satisfies the Markov property

$$
\mathbf{E}^{\mu}\left(g\left(\rho_{j}^{\mu}\right) \mid \sigma\left\{\rho_{0}^{\mu}, \ldots, \rho_{l}^{\mu}\right\}\right)=\mathbf{E}^{\mu}\left(g\left(\rho_{j}^{\mu}\right) \mid \sigma\left\{\rho_{l}^{\mu}\right\}\right) \quad \forall l \leq j \leq k .
$$




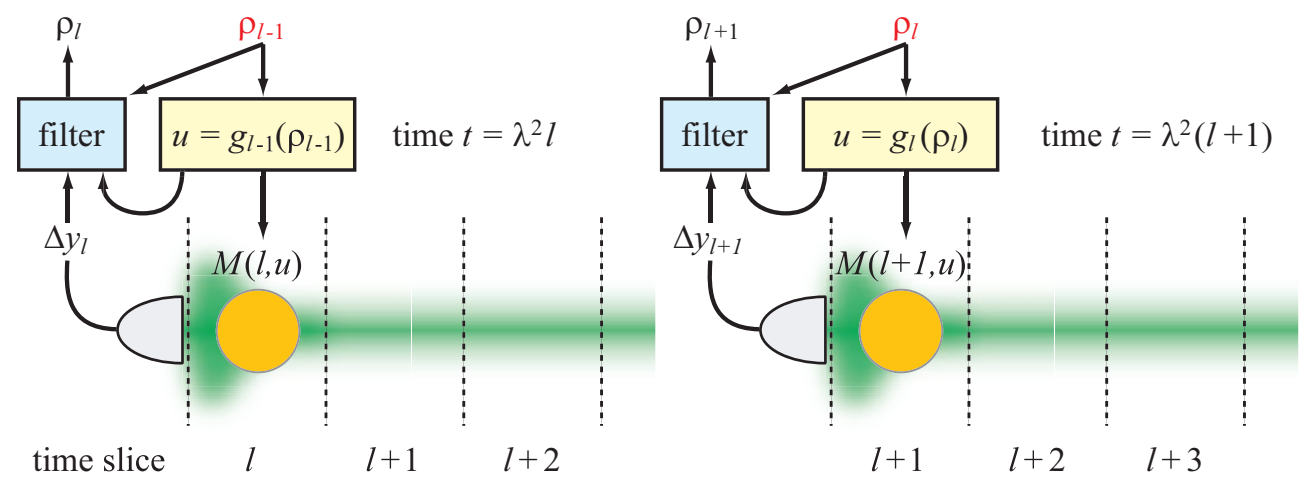

Fig. 7.I The operation of a separated feedback control. The only quantity stored by the controller at the beginning of time step $l$ is the filter state in the previous time step $\rho_{l-1}$. The control $u_{l}$ is calculated using a deterministic function $g_{l}$ and is fed back to the atom through an actuator. Simultaneously, time slice $l$ of the field interacts with the atom. We subsequently detect the field, which gives rise to the observation $\Delta y_{l}$. The filter uses $\Delta y_{l}$ and $u_{l}$ to update the filter state to $\rho_{l}$. The procedure then repeats in time step $l+1$ with $\rho_{l}$ as basis for the control.

8. Optimal Control. Now that we have described repeated interactions with feedback and have obtained filtering equations in this context, the remaining question is, how do we choose the control strategy $\mu$ to achieve a particular control goal? In this section we approach this problem using optimal control theory, where the control goal is expressed as the desire to minimize a certain cost function. Dynamic programming allows us to construct a control strategy and to verify its optimality. For an introduction to optimal stochastic control we refer to, e.g., [60, 62, 35, 4, 14].

In optimal control theory, the control objective is expressed in terms of a cost function $J(\mu)$. In this paper we mainly consider the cost function

$$
J(\mu)=\mathbb{P}\left[\sum_{l=1}^{k} j_{l-1}^{\mu}\left(Q\left(\check{\mathfrak{u}}_{l}\right)\right) \Delta t(l)+j_{k}^{\mu}(K)\right] .
$$

This cost is defined in terms of the quantities $Q: \mathfrak{U} \rightarrow \mathscr{M}$ and $K \in \mathscr{M}$, with $K$ and $Q(u)$ nonnegative and self-adjoint for all $u \in \mathfrak{U}$. Large values of these penalty quantities correspond to undesirable behavior, while small values correspond to the desirable behavior being sought. The penalty $Q(u)$ will be accumulated at each time step, contributing to a running cost. For example, it may take the form $Q(u)=$ $Q+c(u)$, where $Q \in \mathscr{M}$ penalizes deviation from a desired state and the function $c: \mathfrak{U} \rightarrow[0, \infty)$ penalizes the control effort. $K$ is a terminal cost incurred at the end of the time horizon. Ultimately the goal is to find, if possible, an admissible control strategy $\mu$ that minimizes the cost $J(\mu)$

Remark 8.1. Note that the cost $J(\mu)$ is defined on a fixed time interval of length $k$. We could also consider this problem on an infinite time horizon, and use either discounted or average cost per unit time extensions of (8.1) (without the terminal cost $K$ ). Though the theory of this section extends readily to this scenario, we will restrict ourselves to the cost $J(\mu)$ for concreteness.

The key step needed to apply dynamic programming is to express the cost function in terms of a filtered quantity. This will be discussed further in section 8.2 , where 
we describe the concept of information state. The following calculation, which makes use of properties of conditional expectations, the spectral theorem, and Lemma 7.7, expresses the cost (8.1) in terms of the filter (7.9):

$$
\begin{aligned}
J(\mu) & =\mathbb{P}\left[\sum_{l=1}^{k} \mathbb{P}\left[j_{l-1}^{\mu}\left(Q\left(\check{\mathfrak{u}}_{l}\right)\right) \mid \mathscr{Y}_{l-1}\right] \Delta t(l)+\mathbb{P}\left[j_{k}^{\mu}(K) \mid \mathscr{Y}_{k}\right]\right] \\
& =\mathbb{P}\left[\sum_{l=1}^{k} \pi_{l-1}^{\mu}\left[Q\left(\check{\mathfrak{u}}_{l}\right)\right] \Delta t(l)+\pi_{k}^{\mu}[K]\right] \\
& =\mathbf{E}^{\mu}\left[\sum_{l=1}^{k} \iota\left(\pi_{l-1}^{\mu}\left[Q\left(\check{\mathfrak{u}}_{l}\right)\right]\right) \Delta t+\iota\left(\pi_{k}^{\mu}[K]\right)\right] \\
& =\mathbf{E}^{\mu}\left[\sum_{l=1}^{k} \operatorname{Tr}\left[\rho_{l-1}^{\mu} Q\left(u_{l}^{\mu}\right)\right] \Delta t+\operatorname{Tr}\left[\rho_{k}^{\mu} K\right]\right] .
\end{aligned}
$$

Here $\mathbf{E}^{\mu}$ is the $\mathbf{P}^{\mu}$-expectation on the probability space $\left(\Omega^{\mu}, \mathcal{F}^{\mu}, \mathbf{P}^{\mu}\right)$ obtained by applying the spectral theorem to $\mathscr{Y}_{k}^{\mu}$. We retain the superscript ${ }^{\mu}$ to emphasize that the observation algebra is control-dependent; $Y^{\mu}(l)$ and $Y^{\nu}(l)$ need not commute!

The final form of the cost is now a classical cost function for the classical recursion (7.9); in other words, we have reduced the control problem to a problem of classical optimal control for the classical controlled Markov process (7.9). Hence we can apply almost directly the ordinary dynamic programming methodology, which we will do in the next subsection. We only need to take care to use different probability spaces $\Omega^{\mu}, \Omega^{\nu}$ for different control strategies, as the corresponding observations need not commute; this does not present any additional difficulties, however.

In order to implement the dynamic programming method, we will use the fact that the conditional distribution of $\Delta y_{l}^{\mu}$ given $\Delta y_{1}^{\mu}, \ldots, \Delta y_{l-1}^{\mu}$ can be evaluated explicitly. This proceeds in a manner similar to the proof of Lemma 5.11, but taking into account the dependence of the coefficients on the controls using Lemma 7.7.

Lemma 8.2. For any admissible strategy $\mu \in \mathfrak{K}$ we have

$$
\mathbf{P}^{\mu}\left[\Delta y_{l}= \pm \lambda \mid \mathcal{Y}_{l-1}^{\mu}\right]=p\left(\Delta y= \pm \lambda ; \rho_{l-1}^{\mu}, u_{l}^{\mu}\right),
$$

where

$$
p(\Delta y= \pm \lambda ; \rho, u)=\frac{1}{2} \pm \frac{\lambda}{2} \operatorname{Tr}[\overline{\mathcal{J}}(\rho, u)]
$$

depends only on the previous filter state and the control value being applied, and in particular is independent of the feedback strategy $\mu$.

Before we proceed, let us fix some notation. In the following we will encounter separated control strategies $\mu, \mu^{*}, \bar{\mu} \in \mathfrak{K}_{S}$. We will denote the corresponding control functions by $\mu=\left\{g_{0}(\rho), \ldots, g_{k-1}(\rho)\right\}, \mu^{*}=\left\{g_{0}^{*}(\rho), \ldots, g_{k-1}^{*}(\rho)\right\}$, and $\bar{\mu}=$ $\left\{\bar{g}_{0}(\rho), \ldots, \bar{g}_{k-1}(\rho)\right\}$. We will also denote by $\mu \in \mathfrak{K}$ an arbitrary admissible strategy, in which case the corresponding feedback process is always denoted by $u_{l}^{\mu}$.

8.I. Dynamic Programming. The goal of this section is to give a brief introduction to dynamic programming. The main results we will need are the dynamic programming equation or Bellman equation (8.6), which allows us to construct explicitly a candidate optimal control strategy in separated form, and the verification 
Lemma 8.4, which verifies that the strategy constructed through the dynamic programming equation is indeed optimal. As motivation for the Bellman equation we will first prove the "converse" of the verification lemma: if an optimal separated strategy exists, then the Bellman equation follows. The Bellman equation can also be introduced in a more general context without this assumption, and without any reference to separated strategies; this will be discussed briefly at the end of this section.

Let us begin by considering a separated control strategy $\mu \in \mathfrak{K}_{S}$. A central idea in dynamic programming is that one should consider separately the cost incurred over different time intervals within the total control interval $0, \ldots, k$. To this end, let us introduce the cost-to-go

$$
W_{l}(\mu, \rho)=\mathbf{E}^{\mu}\left[\sum_{i=l+1}^{k} \operatorname{Tr}\left[\rho_{i-1}^{\mu} Q\left(u_{i}^{\mu}\right)\right] \Delta t+\operatorname{Tr}\left[\rho_{k}^{\mu} K\right] \mid \rho_{l}^{\mu}=\rho\right]
$$

The quantity $W_{l}(\mu, \rho)$ is the cost incurred by the control $\mu$ over the interval $l, \ldots, k$, given that $\rho_{l}^{\mu}=\rho$. Note in particular that $W_{0}\left(\mu, \rho_{0}\right)=J(\mu)$. It is straightforward to obtain a recursion for $W_{l}(\mu, \rho)$ using the Markov property of the controlled filter:

$$
\begin{aligned}
W_{l}(\mu, \rho) & =\mathbf{E}^{\mu}\left[\operatorname{Tr}\left[\rho_{l}^{\mu} Q\left(u_{l+1}^{\mu}\right)\right] \Delta t+W_{l+1}\left(\mu, \rho_{l+1}^{\mu}\right) \mid \rho_{l}^{\mu}=\rho\right] \\
& =\operatorname{Tr}\left[\rho Q\left(g_{l}(\rho)\right)\right] \Delta t+\sum_{\Delta y= \pm \lambda} p\left(\Delta y ; \rho, g_{l}(\rho)\right) W_{l+1}\left(\mu, \Gamma\left(\rho, g_{l}(\rho), \Delta y\right)\right),
\end{aligned}
$$

with the terminal condition $W_{k}(\mu, \rho)=\operatorname{Tr}[\rho K]$.

The goal of optimal control theory is to find, if possible, a control strategy $\mu^{*}$ that minimizes $J(\mu)$. For the time being, let us suppose that such a strategy exists within the class of separated controls, i.e., that $\mu^{*} \in \mathfrak{K}_{S}$ is such that $J\left(\mu^{*}\right) \leq J(\mu)$ for any $\mu \in \mathfrak{K}_{S}$. It is not unnatural to expect that the same strategy $\mu^{*}$ also minimizes $W_{l}(\mu, \rho)$ for any $l$ and $\rho$ : if the strategy $\mu^{*}$ is optimal over the full time interval $0, \ldots, k$, then it should also be optimal over any subinterval $l, \ldots, k$. If we assume that this is indeed the case, then we can simplify the expression for $W_{l}\left(\mu^{*}, \rho\right)$ :

$$
\begin{aligned}
& W_{l}\left(\mu^{*}, \rho\right)= \inf _{\mu \in \mathfrak{K}_{S}} W_{l}(\mu, \rho) \\
&= \inf _{g_{l}, \ldots, g_{k}}\left[\operatorname{Tr}\left[\rho Q\left(g_{l}(\rho)\right)\right] \Delta t+\sum_{\Delta y= \pm \lambda} p\left(\Delta y ; \rho, g_{l}(\rho)\right) W_{l+1}\left(\mu, \Gamma\left(\rho, g_{l}(\rho), \Delta y\right)\right)\right] \\
&=\inf _{g_{l}}\left[\operatorname{Tr}\left[\rho Q\left(g_{l}(\rho)\right)\right] \Delta t+\inf _{g_{l+1}, \ldots, g_{k}} \sum_{\Delta y= \pm \lambda} p\left(\Delta y ; \rho, g_{l}(\rho)\right) W_{l+1}\left(\mu, \Gamma\left(\rho, g_{l}(\rho), \Delta y\right)\right)\right] \\
&=\inf _{g_{l}}\left[\operatorname{Tr}\left[\rho Q\left(g_{l}(\rho)\right)\right] \Delta t+\sum_{\Delta y= \pm \lambda} p\left(\Delta y ; \rho, g_{l}(\rho)\right) \inf _{\mu \in \mathfrak{K}_{S}} W_{l+1}\left(\mu, \Gamma\left(\rho, g_{l}(\rho), \Delta y\right)\right)\right] \\
&=\inf _{g_{l}}\left[\operatorname{Tr}\left[\rho Q\left(g_{l}(\rho)\right)\right] \Delta t+\sum_{\Delta y= \pm \lambda} p\left(\Delta y ; \rho, g_{l}(\rho)\right) W_{l+1}\left(\mu^{*}, \Gamma\left(\rho, g_{l}(\rho), \Delta y\right)\right)\right] .
\end{aligned}
$$

The following lemma makes these ideas precise. 
Lemma 8.3 (dynamic programming equation). Suppose $\mu^{*} \in \mathfrak{K}_{S}$ is optimal in $\mathfrak{K}_{S}$, i.e., $J\left(\mu^{*}\right) \leq J(\mu)$ for any $\mu \in \mathfrak{K}_{S}$, and define $V_{l}(\rho)=W_{l}\left(\mu^{*}, \rho\right)$. Then

$$
\begin{aligned}
V_{l}(\rho) & =\inf _{u \in \mathfrak{U}}\left[\operatorname{Tr}[\rho Q(u)] \Delta t+\sum_{\Delta y= \pm \lambda} p(\Delta y ; \rho, u) V_{l+1}(\Gamma(\rho, u, \Delta y))\right], \quad l<k, \\
V_{k}(\rho) & =\operatorname{Tr}[\rho K]
\end{aligned}
$$

for all $l$ and any $\rho$ that is reachable under $\mu^{*}$ in the sense that $\mathbf{P}^{\mu^{*}}\left(\rho_{l}^{\mu^{*}}=\rho\right)>0$. Moreover, the infimum in (8.6) is attained at $u=g_{l}^{*}(\rho)$.

Proof. In view of the discussion above, it suffices to show that the conditions of the lemma imply that $\mu^{*}$ minimizes $W_{l}(\mu, \rho)$, i.e., that $W_{l}\left(\mu^{*}, \rho\right) \leq W_{l}(\mu, \rho)$ for any $\mu \in \mathfrak{K}_{S}, l=0, \ldots, k$, and any reachable $\rho$. To this end, let us suppose that this statement holds for time step $l+1$. Then by (8.5)

$$
\operatorname{Tr}\left[\rho Q\left(g_{l}(\rho)\right)\right] \Delta t+\sum_{\Delta y= \pm \lambda} p\left(\Delta y ; \rho, g_{l}(\rho)\right) W_{l+1}\left(\mu^{*}, \Gamma\left(\rho, g_{l}(\rho), \Delta y\right)\right) \leq W_{l}(\mu, \rho)
$$

for any $\mu \in \mathfrak{K}_{S}$ and reachable $\rho$. Note that the left-hand side is precisely $W_{l}\left(\mu^{\prime}, \rho\right)$, where $\mu^{\prime}$ is the control strategy that coincides with $\mu$ at time $l$ and with $\mu^{*}$ at all other times (this follows as $W_{l+1}\left(\mu^{*}, \rho\right)$ depends only on the control functions at times $l+1, \ldots, k)$. We would like to show that the left-hand side is bounded from below by

$$
W_{l}\left(\mu^{*}, \rho\right)=\operatorname{Tr}\left[\rho Q\left(g_{l}^{*}(\rho)\right)\right] \Delta t+\sum_{\Delta y= \pm \lambda} p\left(\Delta y ; \rho, g_{l}^{*}(\rho)\right) W_{l+1}\left(\mu^{*}, \Gamma\left(\rho, g_{l}^{*}(\rho), \Delta y\right)\right)
$$

for all reachable $\rho$. Suppose that this is not the case. We define a new control strategy $\bar{\mu} \in \mathfrak{K}_{S}$ as follows. At time $l$, we set

$$
\bar{g}_{l}(\rho)= \begin{cases}g_{l}^{*}(\rho), & W_{l}\left(\mu^{*}, \rho\right) \leq W_{l}\left(\mu^{\prime}, \rho\right), \\ g_{l}(\rho) & \text { for all other } \rho .\end{cases}
$$

For any time $i \neq l$, we let $\bar{\mu}$ coincide with $\mu^{*}$, i.e., $\bar{g}_{i}(\rho)=g_{i}^{*}(\rho)$ for $i \neq l$. Clearly,

$$
\begin{aligned}
& W_{l}(\bar{\mu}, \rho)=\operatorname{Tr}\left[\rho Q\left(\bar{g}_{l}(\rho)\right)\right] \Delta t \\
& \quad+\sum_{\Delta y= \pm \lambda} p\left(\Delta y ; \rho, \bar{g}_{l}(\rho)\right) W_{l+1}\left(\mu^{*}, \Gamma\left(\rho, \bar{g}_{l}(\rho), \Delta y\right)\right) \leq W_{l}\left(\mu^{*}, \rho\right),
\end{aligned}
$$

with strict inequality for some reachable $\rho$. But then

$$
J\left(\mu^{*}\right)=\mathbf{E}^{\mu^{*}}\left[\sum_{i=1}^{l} \operatorname{Tr}\left[\rho_{i-1}^{\mu^{*}} Q\left(g_{i-1}^{*}\left(\rho_{i-1}\right)\right)\right] \Delta t+W_{l}\left(\mu^{*}, \rho_{l}^{\mu^{*}}\right)\right] \geq J(\bar{\mu}),
$$

which contradicts optimality of $\mu^{*}$. Hence evidently $W_{l}\left(\mu^{*}, \rho\right) \leq W_{l}(\mu, \rho)$ also for time step $l$. It remains to notice that the statement holds trivially for $l=k$ as $W_{k}(\mu, \rho)=\operatorname{Tr}[\rho K]$ is independent of $\mu$, and hence for any $l=0, \ldots, k$ by induction.

Returning to the statement of the lemma, (8.6) can now be obtained as above, and the fact that the infimum is attained at $g_{l}^{*}(\rho)$ follows directly if we compare (8.6) with the expression for $W_{l}\left(\mu^{*}, \rho\right)$ given by $(8.5)$. Hence the proof is complete.

Let us now consider (8.6) without assuming that an optimal control $\mu^{*}$ exists. As a backwards-in-time recursion, this expression makes sense without any reference to 
$\mu^{*}$. $V_{l}(\rho)$ is uniquely defined for any $l=0, \ldots, k$ and $\rho$ : after all, the cost quantities $\operatorname{Tr}[\rho Q(u)]$ and $\operatorname{Tr}[\rho K]$ are bounded from below, so that the infimum exists and is uniquely defined at every step in the recursion starting from the terminal time $k$.

Equation (8.6) is called the dynamic programming equation or Bellman equation. By Lemma 8.3, we find that if an optimal control $\mu^{*} \in \mathfrak{K}_{S}$ exists, then it must be the case that $V_{l}(\rho)=W_{l}\left(\mu^{*}, \rho\right)$ and that all the infima in the recursion are attained; moreover, in this case an optimal control can be recovered by choosing the control functions $g_{l}(\rho)$ to be minimizers at every step in the Bellman recursion.

One could now ask whether the converse also holds true. Suppose that in the Bellman recursion all the infima turn out to be attained. Then we can define a separated control strategy $\mu^{*}$ by setting

$$
g_{l}^{*}(\rho) \in \underset{u \in \mathfrak{U}}{\operatorname{argmin}}\left[\operatorname{Tr}[\rho Q(u)] \Delta t+\sum_{\Delta y= \pm \lambda} p(\Delta y ; \rho, u) V_{l+1}(\Gamma(\rho, u, \Delta y))\right] .
$$

The question is, does this imply that $\mu^{*}$ is optimal? This is indeed the case, as we will show in the following lemma. Note that this immediately gives a constructive way of finding optimal controls, which is precisely the main idea of dynamic programming.

LEMMA 8.4 (verification and separation). Suppose that all the infima in the Bellman equation are attained. Then any control $\mu^{*}$ defined by (8.7) is optimal in $\mathfrak{K}$, i.e., $J\left(\mu^{*}\right) \leq J(\mu)$ for any $\mu \in \mathfrak{K}$. Moreover, $V_{l}(\rho)=W_{l}\left(\mu^{*}, \rho\right)$ for any $l$ and all reachable $\rho$, and in particular $V_{0}\left(\rho_{0}\right)=J\left(\mu^{*}\right)$.

Proof. By substituting (8.7) in (8.6) and comparing with (8.5), the last statement is evident. It remains to show that $J\left(\mu^{*}\right) \leq J(\mu)$ for any $\mu \in \mathfrak{K}$. Note that

$$
V_{l}\left(\rho_{l}^{\mu}\right) \leq \operatorname{Tr}\left[\rho_{l}^{\mu} Q\left(u_{l+1}^{\mu}\right)\right] \Delta t+\sum_{\Delta y= \pm \lambda} p\left(\Delta y ; \rho_{l}^{\mu}, u_{l+1}^{\mu}\right) V_{l+1}\left(\Gamma\left(\rho_{l}^{\mu}, u_{l+1}^{\mu}, \Delta y\right)\right)
$$

by (8.6), where we have chosen an arbitrary $\mu \in \mathfrak{K}$. But by Lemma 8.2 , this is

$$
V_{l}\left(\rho_{l}^{\mu}\right) \leq \operatorname{Tr}\left[\rho_{l}^{\mu} Q\left(u_{l+1}^{\mu}\right)\right] \Delta t+\mathbf{E}^{\mu}\left(V_{l+1}\left(\rho_{l+1}^{\mu}\right) \mid \mathcal{Y}_{l}^{\mu}\right) .
$$

By recursing the relation backwards from $l=k-1$, we obtain

$$
V_{0}\left(\rho_{0}\right) \leq \mathbf{E}^{\mu}\left[\sum_{l=1}^{k} \operatorname{Tr}\left[\rho_{l-1}^{\mu} Q\left(u_{l}^{\mu}\right)\right] \Delta t+\operatorname{Tr}\left[\rho_{k}^{\mu} K\right]\right]=J(\mu) .
$$

But we have already established that $V_{0}\left(\rho_{0}\right)=J\left(\mu^{*}\right)$, hence the result follows.

Let us reflect for a moment on what we have achieved. We began by showing that the cost-to-go for any strategy $\mu^{*}$ that is optimal in the class $\mathfrak{K}_{S}$ of separated controls must satisfy the Bellman recursion. Conversely, if the infima in the Bellman equation are all attained, we can construct a control strategy $\mu^{*}$ by solving the Bellman recursion (dynamic programming). We then verified in Lemma 8.4 (the verification lemma) that the strategy thus constructed is indeed optimal, not only in the class of separated controls but in the class $\mathfrak{K}$ of all admissible controls. Combining these two results, we conclude that any strategy that is optimal in $\mathfrak{K}_{S}$ is necessarily optimal within the larger class $\mathfrak{K}$ (Lemma 8.4 is also called the separation lemma for this reason). This shows that the idea of separated controls is very natural, and indeed universal, for this type of control problem.

Remark 8.5. We have not given conditions under which existence of the infima in the Bellman recursion is guaranteed. This can be a delicate issue; see, e.g., [15]. 
However, if $\mathfrak{U}$ is a finite set the infima are trivially attained. This particularly simple case is also the most straightforward to implement on a computer.

Remark 8.6. The results above are formulated only for reachable $\rho$. This is as it should be, as for nonreachable $\rho$ the cost-to-go is defined as a conditional expectation on a set of measure zero and is thus not unique. These issues are irrelevant, however, to the application of the dynamic programming algorithm and the verification lemma, which are the most important results of this section.

Finally, we briefly remark on the case where the infima in the Bellman equation are not attained. It follows from Lemma 8.3 that there cannot exist an optimal separated control in this case. Nonetheless the solution $V_{l}(\rho)$ of the Bellman equation, called the value function, is still a relevant quantity; it can be shown to be the infimum (not minimum!) of the cost-to-go over a suitable class of (not necessarily separated) controls. This characterization can be useful in practice, for example, if we wish to quantify the performance of suboptimal controls.

This approach also provides a different entry point into the theory of dynamic programming than the one we have chosen, providing additional insight into the structure of the theory. One could begin by proving directly that the value function $V_{l}(\rho)$, now defined as the infimum of the cost over the time horizon $l, \ldots, k$, satisfies the dynamic programming equation. This does not require us to assume the existence of an optimal control, and in particular places no a priori preference on the class of separated controls. The only thing that is needed is the fact that the filtering equation is a controlled Markov process, which is key to the entire procedure. Indeed, we have used this property in an essential way in the form of Lemma 8.2. If the infima in the Bellman recursion are attained, then we can construct an explicit separated control strategy as was demonstrated above; verification then proceeds as we have indicated.

We will not further detail this approach here, as we do not need these results in what follows. We refer to [15] for an extensive study of discrete time dynamic programming, or to, e.g., [35] for the continuous time case.

8.2. Information States. The key idea that facilitated the dynamic programming solution to the optimal control problem discussed above was the representation of the cost $J(\mu)$ in terms of the filtered quantity $\rho_{l}^{\mu}$. This is an instance of a general methodology involving information state representations [60, Chapter 6]. An information state is a quantity that is causally computable from information available to the controller, i.e., the observation record and previous choices of control values. The dynamic evolution of the information state is given by an information state filter. Solving a given optimal control problem reduces to expressing the cost function exclusively in terms of a suitable information state, then applying the dynamic programming method. The resulting optimal feedback control will be a separated control relative to the information state filter. In the previous sections we used the density operator $\rho_{l}^{\mu}$ as an information state to represent the cost $J(\mu)$, as indicated in the calculation (8.2), and the filter for this information state was given by (7.9).

The choice of information state for a given problem is not unique. In the case of the cost function $J(\mu)$ defined by (8.1), we could also use the unnormalized conditional density operator $\varrho_{l}^{\mu}$ discussed in section 7.4 as an information state, with the corresponding filter (7.10). To see this, we use the reference probability method and condition on $\mathscr{C}_{l}$ as follows. We begin by defining the state

$$
\mathbb{P}^{0 \mu}[X]=\mathbb{P}\left[U^{\mu}(k) X U^{\mu}(k)^{*}\right], \quad X \in \mathscr{M} \otimes \mathscr{W}_{k},
$$

and we denote the associated classical state, as obtained through the spectral theorem, 
by $\mathbf{P}^{0 \mu}$. Under $\mathbf{P}^{0 \mu}, \Delta y_{1}, \ldots, \Delta y_{k}$ are i.i.d. random variables taking values $\pm \lambda$ with equal probability, and in particular the law of the process $\Delta y$ under $\mathbf{P}^{0 \mu}$ is independent of the feedback control $\mu \in \mathfrak{K}$. Then (cf. (8.2)) we have

$$
\begin{aligned}
& J(\mu)\left.=\mathbb{P}\left[\sum_{l=1}^{k} V^{\mu}(l-1)^{*} Q\left(\check{\mathfrak{u}}_{l}\right) V^{\mu}(l-1) \Delta t(l)+V^{\mu}(k)^{*} K V^{\mu}(k)\right]\right] \\
&= \mathbb{P}\left[\sum_{l=1}^{k} \mathbb{P}\left[V^{\mu}(l-1)^{*} Q\left(\check{\mathfrak{u}}_{l}\right) V^{\mu}(l-1) \mid \mathscr{C}_{l-1}\right] \Delta t(l)+\mathbb{P}\left[V^{\mu}(k)^{*} K V^{\mu}(k) \mid \mathscr{C}_{k}\right]\right] \\
&= \mathbb{P}\left[\sum_{l=1}^{k} U^{\mu}(k) U^{\mu}(k)^{*} \mathbb{P}\left[V^{\mu}(l-1)^{*} Q\left(\check{\mathfrak{u}}_{l}\right) V^{\mu}(l-1) \mid \mathscr{C}_{l-1}\right] U^{\mu}(k) U^{\mu}(k)^{*} \Delta t(l)\right. \\
&\left.+U^{\mu}(k) U^{\mu}(k)^{*} \mathbb{P}\left[V^{\mu}(k)^{*} K V^{\mu}(k) \mid \mathscr{C}_{k}\right] U^{\mu}(k) U^{\mu}(k)^{*}\right]
\end{aligned}
$$

where the change of state operator $V^{\mu}(l)$ was defined in Lemma 7.6. Now recall that $U^{\mu}(k)^{*} \mathbb{P}\left[X \mid \mathscr{C}_{l}\right] U^{\mu}(k)=U^{\mu}(l) * \mathbb{P}\left[X \mid \mathscr{C}_{l}\right] U^{\mu}(l)$; see the proof of the nondemolition property in section 2.5 . Changing to the state $\mathbb{P}^{0 \mu}$, we therefore obtain

$$
\begin{aligned}
J(\mu) & =\mathbb{P}^{0 \mu}\left[\sum_{l=1}^{k} \sigma_{l-1}^{\mu}\left[Q\left(\check{\mathfrak{u}}_{l}\right)\right] \Delta t(l)+\sigma_{k}^{\mu}[K]\right] \\
& =\mathbf{E}^{0 \mu}\left[\sum_{l=1}^{k} \iota\left(\sigma_{l-1}^{\mu}\left[Q\left(\check{\mathfrak{u}}_{l}\right)\right]\right) \Delta t+\iota\left(\sigma_{k}^{\mu}[K]\right)\right] \\
& =\mathbf{E}^{0 \mu}\left[\sum_{l=1}^{k} \operatorname{Tr}\left[\varrho_{l-1}^{\mu} Q\left(u_{l}^{\mu}\right)\right] \Delta t+\operatorname{Tr}\left[\varrho_{k}^{\mu} K\right]\right],
\end{aligned}
$$

where the unnormalized conditional state $\sigma_{l}^{\mu}$ was defined by (7.8) and $\varrho_{l}^{\mu}$ is the associated density matrix. Using this representation, we can define a value function $S_{l}(\varrho)$ and find an optimal control using the alternate dynamic programming equation

$$
\begin{aligned}
S_{l}(\varrho) & =\inf _{u \in \mathfrak{U}}\left[\operatorname{Tr}[\varrho Q(u)] \Delta t+\sum_{\Delta y= \pm \lambda} p^{0}(\Delta y) S_{l+1}(\Sigma(\varrho, u, \Delta y))\right], \quad l<k, \\
S_{k}(\varrho) & =\operatorname{Tr}[\varrho K],
\end{aligned}
$$

where $p^{0}(\Delta y= \pm \lambda)=0.5$. In fact, the optimal control is given by

$$
h_{l}^{*}(\varrho) \in \underset{u \in \mathfrak{U}}{\operatorname{argmin}}\left[\operatorname{Tr}[\varrho Q(u)] \Delta t+\sum_{\Delta y= \pm \lambda} p^{0}(\Delta y) S_{l+1}(\Sigma(\varrho, u, \Delta y))\right] .
$$

This is a separated feedback control relative to the information state filter (7.10).

The conditional state (either normalized or unnormalized) is not the correct choice for every cost function. In 1981 Whittle [82] discovered a different type of information state for optimal control problems with exponential cost functions (risk-sensitive control), which are not solvable using the standard conditional states. Instead, the filter for the corresponding information state depends explicitly on quantities defining

Copyright (c) by SIAM. Unauthorized reproduction of this article is prohibited. 
the cost function, and the optimal feedback control is separated relative to this filter. Following [50] we now explain this briefly in the context of this paper.

In the quantum setting, a risk-sensitive cost can be defined by

$$
J^{\theta}(\mu)=\mathbb{P}\left[C(k)^{*} e^{\theta j_{k}^{\mu}(K)} C(k)\right],
$$

where $\Delta C(l)=\frac{\theta}{2} j_{l-1}^{\mu}\left(Q\left(\check{\mathfrak{u}}_{l-1}\right)\right) C(l-1) \Delta t(l), C(0)=I$, defines the "exponential" running cost, and $\theta>0$ is a fixed real parameter (the risk parameter). Let us now define $\tilde{U}^{\mu}(l)=U^{\mu}(l) C(l)$ (which is not unitary in general), so that

$$
J^{\theta}(\mu)=\mathbb{P}\left[\tilde{U}^{\mu}(k)^{*} e^{\theta K} \tilde{U}^{\mu}(k)\right] .
$$

We can now proceed as in the previous part of this section to express the control cost in terms of an unnormalized filtered quantity. The corresponding filter is not obtained from the usual unitary $U^{\mu}(l)$, however, but from the modified operator $\tilde{U}^{\mu}(l)$. We can obtain a change-of-state operator $\tilde{V}^{\mu}(l)$ as in Lemma 7.6, which gives rise to an information state filter that depends explicitly on the running cost $Q(u)$. We can subsequently express $J^{\theta}(\mu)$ in terms of this filter, and the optimal control problem can then be solved using dynamic programming. We leave the details as an exercise.

In classical stochastic control, the risk-sensitive cost is known to possess improved robustness properties compared to the usual cost $J(\mu)$; in particular, as the risk parameter $\theta$ increases, the optimal performance becomes less sensitive to the details of the underlying model. To what extent these advantages carry over to the quantum case remains to be explored (but see [51]).

8.3. Example. We will give a numerical example of dynamic programming for a particularly simple system - a controlled version of the dispersive interaction model.

The Controlled Quantum Flow. We consider again the dispersive interaction model of section 2.6, but now we add a control input. The controlled repeated interaction matrices are now given by

$$
L_{1}(u)=0, \quad L_{2}(u)=i \sigma_{z}, \quad L_{3}(u)=i u\left(\sigma_{+}-\sigma_{-}\right) .
$$

Such an interaction can be realized in certain systems by applying a magnetic field of strength $u$ to the atom; see, e.g., Figure 1.1, in addition to the usual dispersive interaction with the electromagnetic field. In principle any $u \in \mathbb{R}$ is admissible, i.e., we should take $\mathfrak{U}=\mathbb{R}$. As we will be evaluating the dynamic programming recursion numerically, however, it is more convenient to discretize the admissible controls, i.e., we choose $\mathfrak{U}=\{-K \varepsilon,-(K-1) \varepsilon, \ldots, K \varepsilon\} \subset \mathbb{R}$, where $\varepsilon$ is the discretization step size and $2 K+1$ is the total number of admissible controls.

Our first task is to evaluate the matrices $M^{ \pm,+,-, o}(u)$ in the controlled difference equation. Let us calculate explicitly

$$
M_{l}(u)=\exp \left(\sigma_{z}\left(\Delta A^{*}(l)-\Delta A(l)\right)+u\left(\sigma_{+}-\sigma_{-}\right) \Delta t(l)\right) .
$$

Writing

$$
B(u)=\sigma_{z} \otimes \lambda\left(\sigma_{+}-\sigma_{-}\right)+u\left(\sigma_{+}-\sigma_{-}\right) \otimes \lambda^{2} I=\left(\begin{array}{cccc}
0 & \lambda^{2} u & \lambda & 0 \\
-\lambda^{2} u & 0 & 0 & -\lambda \\
-\lambda & 0 & 0 & \lambda^{2} u \\
0 & \lambda & -\lambda^{2} u & 0
\end{array}\right),
$$


we calculate the matrix exponential

$$
e^{B(u)}=\left(\begin{array}{cccc}
\cos (\lambda w(u)) & u \lambda \frac{\sin (\lambda w(u))}{w(u)} & \frac{\sin (\lambda w(u))}{w(u)} & 0 \\
-u \lambda \frac{\sin (\lambda w(u))}{w(u)} & \cos (\lambda w(u)) & 0 & -\frac{\sin (\lambda w(u))}{w(u)} \\
-\frac{\sin (\lambda w(u))}{w(u)} & 0 & \cos (\lambda w(u)) & u \lambda \frac{\sin (\lambda w(u))}{w(u)} \\
0 & \frac{\sin (\lambda w(u))}{w(u)} & -u \lambda \frac{\sin (\lambda w(u))}{w(u)} & \cos (\lambda w(u))
\end{array}\right)
$$

where we have written $w(u)=\sqrt{1+\lambda^{2} u^{2}}$. Hence we obtain

$$
\begin{aligned}
M_{l}(u)=\frac{\sin (\lambda w(u))}{\lambda w(u)} \sigma_{z} & \left(\Delta A^{*}(l)-\Delta A(l)\right) \\
+ & {\left[\frac{\cos (\lambda w(u))-1}{\lambda^{2}}+\frac{\sin (\lambda w(u))}{\lambda w(u)} u\left(\sigma_{+}-\sigma_{-}\right)\right] \Delta t(l)+I . }
\end{aligned}
$$

We can now immediately determine the coefficients in the quantum stochastic difference equation for the controlled dispersive interaction model:

$$
\begin{array}{ll}
M^{ \pm}(u)=0, & M^{+}(u)=\frac{\sin (\lambda w(u))}{\lambda w(u)} \sigma_{z}, \\
M^{-}(u)=-\frac{\sin (\lambda w(u))}{\lambda w(u)} \sigma_{z}, & M^{\circ}(u)=\frac{\cos (\lambda w(u))-1}{\lambda^{2}}+\frac{\sin (\lambda w(u))}{\lambda w(u)} u\left(\sigma_{+}-\sigma_{-}\right) .
\end{array}
$$

An Invariant Set. The solution $\rho_{l}^{\mu}$ of the filtering recursion is always a density matrix; recall that $\iota\left(\pi_{l}^{\mu}(X)\right)=\operatorname{Tr}\left[\rho_{l}^{\mu} X\right]$ is a positive map with $\pi_{l}^{\mu}(I)=1$, so that $\rho_{l}^{\mu}$ must be a positive matrix and have unit trace. Then the goal of dynamic programming is to calculate the feedback function $g_{l}^{*}(\rho)$ for any time step $l$ and density matrix $\rho$. Unfortunately, even the space of $2 \times 2$ density matrices is rather large; discretization of this space, as would be necessary for computer implementation of the dynamic programming recursion, would require a tremendous number of discretization points. For this reason dynamic programming is computationally expensive, prohibitively so in moderate- to high-dimensional systems where optimal control theory often plays the role of a benchmark rather than a practical solution. In such cases, one is compelled to settle for control designs that are suboptimal, i.e., they do not minimize a cost function. We will briefly explore one such approach in section 9 .

The simple example treated in this section, however, has a feature which significantly simplifies the implementation of dynamic programming. As we will demonstrate shortly, there is an invariant set of density matrices which is parametrized by points on a circle; i.e., if we start the filter somewhere on this circle, it will always remain there. This reduces the dynamic programming algorithm to the calculation of the feedback function $g_{l}^{*}(\rho)$ on the circle. With a sufficiently accurate discretization of the circle, this problem can be solved numerically without too many complications. 
To find the desired invariant set, note that

$$
\begin{aligned}
& \mathcal{L}^{\mu}\left(\sigma_{z}, u\right)=\frac{\sin (2 \lambda w(u))}{\lambda w(u)} u\left(\sigma_{+}+\sigma_{-}\right)-\frac{2 u^{2} \sin ^{2}(\lambda w(u))}{w(u)^{2}} \sigma_{z}, \\
& \mathcal{J}^{\mu}\left(\sigma_{z}, u\right)=\frac{\sin (2 \lambda w(u))}{\lambda w(u)} I \\
& \mathcal{L}^{\mu}\left(\sigma_{+}+\sigma_{-}, u\right)=-\frac{\sin (2 \lambda w(u))}{\lambda w(u)} u \sigma_{z}-\frac{2 \sin ^{2}(\lambda w(u))}{\lambda^{2}}\left(\sigma_{+}+\sigma_{-}\right), \\
& \mathcal{J}^{\mu}\left(\sigma_{+}+\sigma_{-}, u\right)=-\frac{2 u \sin ^{2}(\lambda w(u))}{w(u)^{2}} I \\
& \mathcal{J}^{\mu}(I, u)=\frac{\sin (2 \lambda w(u))}{\lambda w(u)} \sigma_{z}+\frac{2 u \sin ^{2}(\lambda w(u))}{w(u)^{2}}\left(\sigma_{+}+\sigma_{-}\right) .
\end{aligned}
$$

Hence evidently the recursion for $\pi_{l}^{\mu}\left(\sigma_{z}\right)$ and $\pi_{l}^{\mu}\left(\sigma_{+}+\sigma_{-}\right)$forms a closed set of equations. We claim furthermore that $\pi_{l}^{\mu}\left(\sigma_{z}\right)^{2}+\pi_{l}^{\mu}\left(\sigma_{+}+\sigma_{-}\right)^{2}=I$ for all $l$, if this is true for $l=0$. The algebra is a little easier if we consider the unnormalized version; using the discrete Itô rule, we calculate

$$
\begin{aligned}
& \Delta\left(\sigma_{l}^{\mu}(X)^{2}\right) \\
& =\left\{2 \sigma_{l-1}^{\mu}(X) \sigma_{l-1}^{\mu}\left(\mathcal{L}^{\mu}\left(X, u_{l}^{\mu}\right)\right)+\lambda^{2} \sigma_{l-1}^{\mu}\left(\mathcal{L}^{\mu}\left(X, u_{l}^{\mu}\right)\right)^{2}+\sigma_{l-1}^{\mu}\left(\mathcal{J}^{\mu}\left(X, u_{l}^{\mu}\right)\right)^{2}\right\} \Delta t(l) \\
& +2 \sigma_{l-1}^{\mu}\left(X+\lambda^{2} \mathcal{L}^{\mu}\left(X, u_{l}^{\mu}\right)\right) \sigma_{l-1}^{\mu}\left(\mathcal{J}^{\mu}\left(X, u_{l}^{\mu}\right)\right) \Delta Y^{\mu}(l) .
\end{aligned}
$$

A tedious but straightforward calculation shows that

$\Delta\left[\sigma_{l}^{\mu}\left(\sigma_{z}\right)^{2}+\sigma_{l}^{\mu}\left(\sigma_{+}+\sigma_{-}\right)^{2}-\sigma_{l}^{\mu}(I)^{2}\right]=\vartheta_{u, \lambda}\left[\sigma_{l-1}^{\mu}\left(\sigma_{z}\right)^{2}+\sigma_{l-1}^{\mu}\left(\sigma_{+}+\sigma_{-}\right)^{2}-\sigma_{l-1}^{\mu}(I)^{2}\right] \Delta t(l)$,

where $\vartheta_{u, \lambda}$ is a complicated function of $u$ and $\lambda$. Hence if $\pi_{0}^{\mu}\left(\sigma_{z}\right)^{2}+\pi_{0}^{\mu}\left(\sigma_{+}+\sigma_{-}\right)^{2}=I$, it follows that $\pi_{l}^{\mu}\left(\sigma_{z}\right)^{2}+\pi_{l}^{\mu}\left(\sigma_{+}+\sigma_{-}\right)^{2}=I$ for all $l$.

Now let $\tilde{\rho}$ be any density matrix. The remaining insight we need is that if we are given $x, z \in \mathbb{R}$ such that $\operatorname{Tr}\left[\tilde{\rho}\left(\sigma_{+}+\sigma_{-}\right)\right]=x, \operatorname{Tr}\left[\tilde{\rho} \sigma_{z}\right]=z$, and $x^{2}+z^{2}=1$, then this uniquely determines $\tilde{\rho}$. To see this, let us write without loss of generality $x=\sin \theta$ and $z=\cos \theta$. Using the constraints $\operatorname{Tr}\left[\tilde{\rho}\left(\sigma_{+}+\sigma_{-}\right)\right]=\sin \theta, \operatorname{Tr}\left[\tilde{\rho} \sigma_{z}\right]=\cos \theta, \operatorname{Tr} \rho=1$, and $\rho=\rho^{*}$, we easily find that

$$
\tilde{\rho}=\left(\begin{array}{cc}
\frac{1}{2}+\frac{1}{2} \cos \theta & \frac{1}{2} \sin \theta+i \beta \\
\frac{1}{2} \sin \theta-i \beta & \frac{1}{2}-\frac{1}{2} \cos \theta
\end{array}\right) \quad \text { for some } \quad \beta \in \mathbb{R} .
$$

But we can explicitly calculate the eigenvalues of this matrix as $\frac{1}{2}\left(1 \pm\left(1+4 \beta^{2}\right)^{1 / 2}\right)$, so that the remaining requirement for the density matrix $\rho \geq 0$ implies that $\beta=0$. Hence we conclude that the "circle" of density matrices

$$
S^{1}=\left\{\left(\begin{array}{cc}
\frac{1}{2}+\frac{1}{2} \cos \theta & \frac{1}{2} \sin \theta \\
\frac{1}{2} \sin \theta & \frac{1}{2}-\frac{1}{2} \cos \theta
\end{array}\right): \theta \in[0,2 \pi)\right\},
$$

parametrized by the angle $\theta$, is invariant under the filtering equation for our controlled quantum flow in the sense that $\rho_{l}^{\mu} \in S^{1}$ for all $l$ if $\rho_{0} \in S^{1}$. We can thus restrict the dynamic programming recursion to this set, which yields a feedback control law on the circle of the form $g_{l}(\theta)$.

Copyright $@$ by SIAM. Unauthorized reproduction of this article is prohibited. 

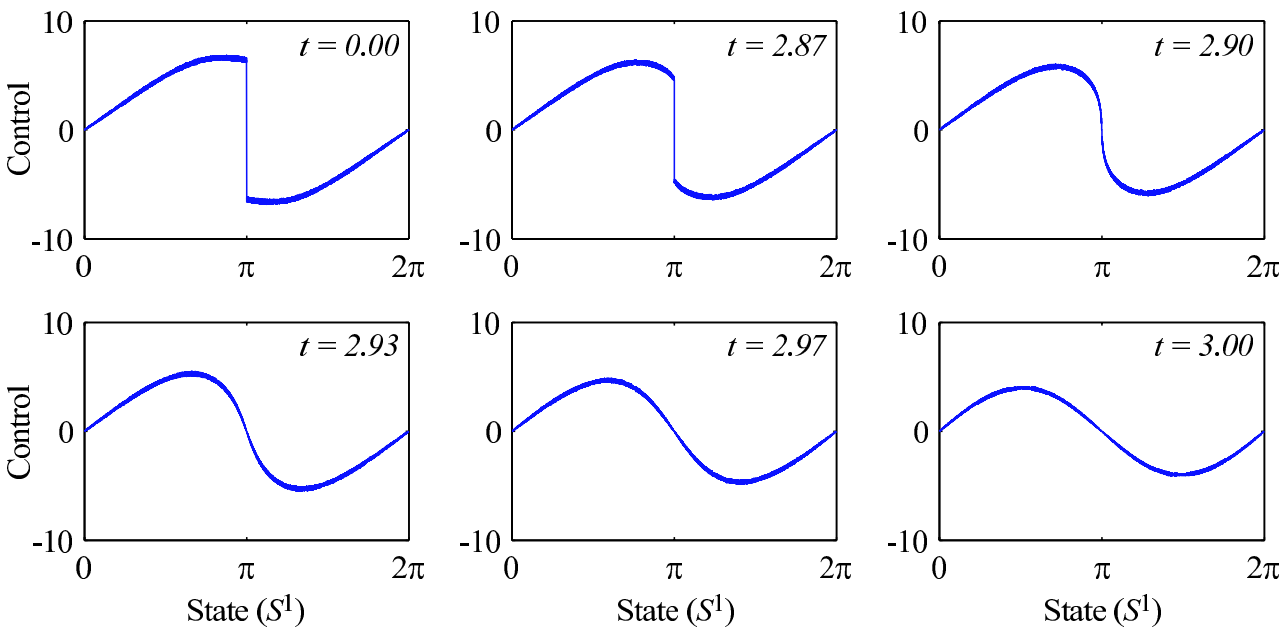

Fig. 8.I The optimal control function $g_{l}^{*}(\theta)$, for selected $l$, obtained by applying the dynamic programming algorithm to the cost function (8.9). During the major portion of the control interval, the feedback function is essentially equal to the first plot; the control passes through zero at the state of maximal energy and has a break at the point of minimal energy. Close to the terminal time, the optimal control varies a little to accommodate the terminal cost.

Dynamic Programming. We are now in a position to solve the dynamic programming algorithm numerically. To this end, we have discretized the circle into a set of $10^{5}$ equidistant points, and we have discretized the control set $\mathfrak{U}$ into 400 equidistant points in the interval $[-10,10]$. As in the previous simulations, we have chosen $\lambda^{-2}=300$ and a terminal time of 3 (i.e., $k=900$ ).

As a first control goal, suppose we would like to maximize the expected energy, i.e., we would like to drive $\operatorname{Tr}\left[\rho_{l}^{\mu} \sigma_{z}\right]$ to +1 . To this end, we use the cost (8.1) with

$$
\begin{aligned}
Q(u) & =C u^{2}+D\left(I-\sigma_{z}\right), \\
K & =I-\sigma_{z} .
\end{aligned}
$$

The first term in $Q(u)$ penalizes the use of large control strengths, which is necessary in any practical feedback loop. The second term tries to minimize $1-\operatorname{Tr}\left[\rho_{l}^{\mu} \sigma_{z}\right]$ during the running time of the system, whereas the terminal cost tries to minimize the terminal value of $1-\operatorname{Tr}\left[\rho_{l}^{\mu} \sigma_{z}\right]$. The constants $C, D>0$ determine the relative weights attributed to these control goals. As an example, we have chosen $C=0.25, D=5$. The corresponding optimal feedback function $g_{l}(\theta)$ is plotted for several times $l$ in Figure 8.1. Note that the control passes through zero at the state of maximal energy $\theta=0$, whereas the break at $\theta=\pi$ drives the system away from the state of minimal energy. This is not unexpected, as both $\theta=\{0, \pi\}$ are fixed points of the filter (see section 5.6); the break in the control destabilizes the undesired minimal energy state.

As a second example, suppose we would like to drive the system close to the point where $\operatorname{Tr}\left[\rho_{l}^{\mu} \sigma_{z}\right]=\operatorname{Tr}\left[\rho_{l}^{\mu} \sigma_{x}\right]=2^{-1 / 2}$, i.e., $\theta=\pi / 4$. This point is uniquely characterized by $\operatorname{Tr}\left[\rho_{l}^{\mu}\left(\sigma_{z}+\sigma_{x}\right)\right]=\sqrt{2}$, so we choose

$$
\begin{aligned}
Q(u) & =C u^{2}+D(I-X), \\
K & =I-X,
\end{aligned}
$$

where $X=2^{-1 / 2}\left(\sigma_{x}+\sigma_{z}\right)$ and, for example, $C=0.25$ and $D=5$. The optimal 

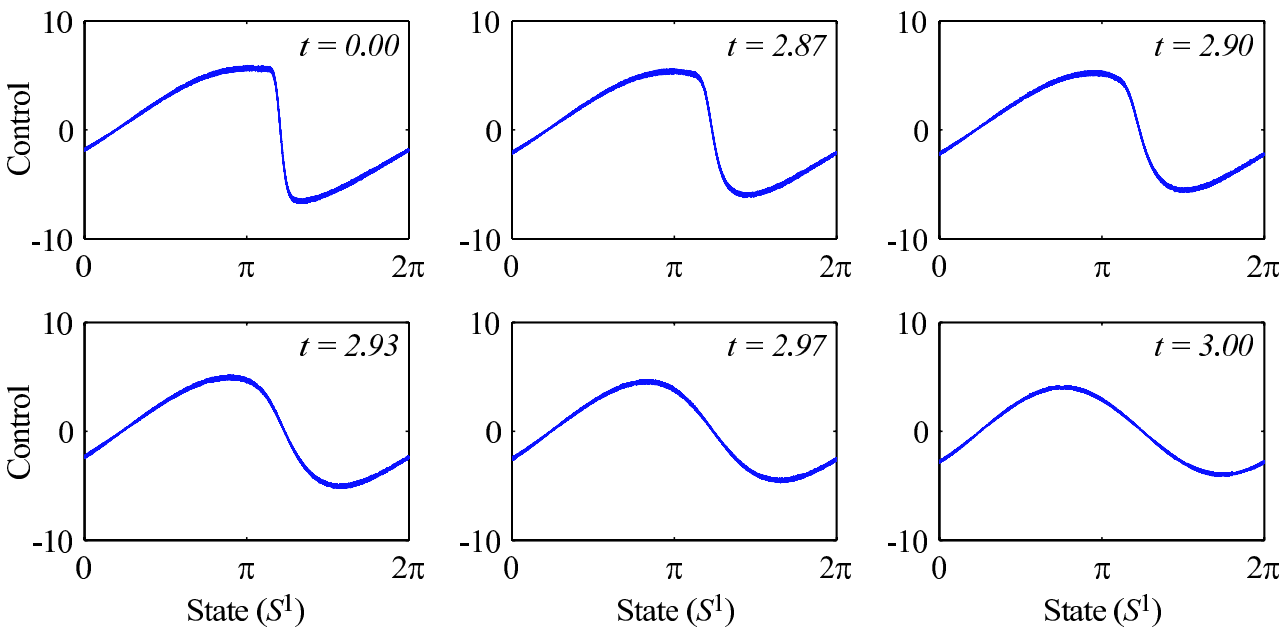

Fig. 8.2 The optimal control function $g_{l}^{*}(\theta)$ for the cost (8.10). As before, the feedback function is essentially equal to the first plot during the major portion of the control interval, and the control passes through zero at the target state. In this case, however, the control function is not discontinuous at the point of the circle opposite to the target state.
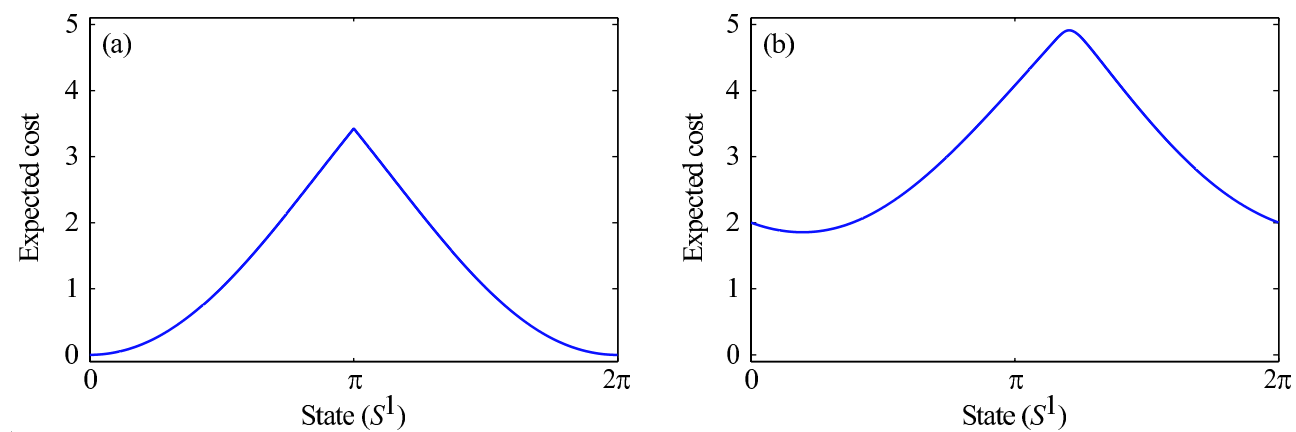

Fig. 8.3 The cost $J\left(\mu^{*}\right)$, as a function of $\rho_{0} \in S^{1}$, for the optimal control strategy $\mu^{*}$ minimizing the cost (a) (8.9) and (b) (8.10). The cost is simply obtained as the final value function in the dynamic programming recursion, $J\left(\mu^{*}\right)\left(\rho_{0}\right)=V_{0}\left[\rho_{0}\right]$. In case (a) the cost is zero at $\theta=0$ : after all, the target point is a fixed point of the filter, so no cost is accumulated if we start at the target point. This is not the case in (b), where the target point is not a fixed point.

feedback function for this case is plotted in Figure 8.2. Once again, the feedback function passes through zero at the target point $\theta=\pi / 4$. However, note that the function is no longer singular; as the opposite point on the circle is not a fixed point of the filter in this case, it is evidently more efficient to let the filter drive itself toward the target point without expending additional control effort.

Finally we have plotted the optimal cost $J\left(\mu^{*}\right)$ as a function of the initial state $\rho \in$ $S^{1}$ in Figure 8.3. The cost is easily obtained as a byproduct of dynamic programming, as it is simply given by the value function at time zero. Note that in the case of our first example, zero cost is possible: as the target $\theta=0$ is a fixed point of the filter, no cost is accumulated if the system is initially in its state of maximal energy. In our 
second example, on the other hand, this is not the case: even if we start at the target state $\theta=\pi / 4$, the filter will fluctuate around this point and a total cost (just under $\left.J\left[\mu^{*}\right]=2\right)$ is accumulated over the control interval.

9. Lyapunov Control. Many control design methods other than optimal control have been developed and are applied in control engineering, for a variety of reasons. Indeed, the applicability of dynamic programming is limited by computational complexity for use in low-dimensional problems or in situations where explicit solutions are available. Among the alternative methods are Lyapunov design methods, and the purpose of this section is to demonstrate their use in our quantum context.

Consider, for example, the following scenario. Recall that the dispersively interacting atom, when untouched by the experimenter, has constant but unknown energy; our control goal is to drive the energy to a particular value of our choice, say, $+\hbar \omega_{0} / 2$. In the absence of sharp time constraints this problem is not uniquely expressed as an optimal control problem: what cost should one choose? All we want to achieve is that $\mathbb{P}\left(j_{l}(H)\right) \rightarrow \hbar \omega_{0} / 2$ for large $l$, a much more modest control goal than the absolute minimization of a particular cost function. Hence dynamic programming is unnecessarily complicated for the problem at hand, and we can resort to a simpler method for control design. In this section we will design a controller for the control problem described above through a very simple Lyapunov function method, similar in spirit to [80]. The simplicity of the method is its chief feature: not only will we find a feedback function that works, but the resulting feedback function is also of a very simple form and is easily implementable.

As before, we can easily express the control goal in terms of the filter $\mathbb{P}\left(j_{l}(H)\right)=$ $\mathbb{P}\left(\pi_{l}(H)\right) \rightarrow \hbar \omega_{0} / 2$ for large $l$. We will design a separated control that achieves this goal. The Markov property of the filter with separated controls is key to the procedure: it allows us to treat the problem entirely at the level of the filter, without reference to the original repeated interaction model.

9.I. Lyapunov Stability. The main tool is the following Lyapunov theorem for Markov processes, taken directly from [62].

ThEOREM 9.1 (Lyapunov theorem). Let $x_{l}$ be a (classical) Markov process. Suppose there exists a nonnegative (Lyapunov) function $V(x) \geq 0$ that satisfies

$$
\mathbf{E}\left(\Delta V\left(x_{l}\right) \mid \sigma\left\{x_{l-1}\right\}\right)=\mathbf{E}\left(V\left(x_{l}\right) \mid \sigma\left\{x_{l-1}\right\}\right)-V\left(x_{l-1}\right)=-k\left(x_{l-1}\right) \leq 0 \quad \forall l
$$

where $k(x) \geq 0$ is another nonnegative function. Then $k\left(x_{l}\right) \rightarrow 0$ as $l \rightarrow \infty$ a.s.

Proof. Fix some $n>0$. As $V(x)$ is a nonnegative function, clearly

$$
V\left(x_{0}\right) \geq V\left(x_{0}\right)-\mathbf{E}\left(V\left(x_{n}\right)\right) .
$$

But by the condition of the theorem, we obtain

$$
\mathbf{E}\left(V\left(x_{n}\right)\right)=\mathbf{E}\left(V\left(x_{n-1}\right)-k\left(x_{n-1}\right)\right)=\mathbf{E}\left(V\left(x_{n-2}\right)-k\left(x_{n-2}\right)-k\left(x_{n-1}\right)\right)=\cdots
$$

Iterating this procedure, we obtain

$$
V\left(x_{0}\right) \geq \mathbf{E}\left(\sum_{l=0}^{n-1} k\left(x_{l}\right)\right)
$$

Copyright (C) by SIAM. Unauthorized reproduction of this article is prohibited. 
As this holds for any $n$ and as $V\left(x_{0}\right)<\infty$, the right-hand side is finite for any $n$. But

$$
\sum_{l=0}^{\infty} \mathbf{P}\left(k\left(x_{l}\right) \geq \varepsilon\right) \leq \frac{1}{\varepsilon} \mathbf{E}\left(\sum_{l=0}^{\infty} k\left(x_{l}\right)\right)<\infty \quad \forall \varepsilon>0
$$

by Chebyshev's inequality, so the Borel-Cantelli lemma gives $k\left(x_{l}\right) \rightarrow 0$ a.s.

Remark 9.2. Previously we did everything on a fixed time horizon $l=0, \ldots, k$. Now, however, we are considering what happens as $l \rightarrow \infty$, so technically a little more care is needed. The reader should convince himself that not much changes in this case. In particular, there is no need to deal directly with an infinite-dimensional algebra $\mathscr{W}_{\infty}=\mathscr{M}^{\otimes \infty}$ corresponding to an infinite number of time slices of the electromagnetic field. Rather, one can use the natural embedding $i: \mathscr{M} \otimes \mathscr{W}_{k} \rightarrow \mathscr{M} \otimes \mathscr{W}_{k+1}, i(X)=$ $X \otimes I$, and the sequence of probability spaces $\Omega^{k} \subset \Omega^{k+1}$ obtained by applying the spectral theorem to the corresponding observation algebras, to give meaning to infinite time limits without functional analytic complications (provided the time step $\lambda$ and the repeated interaction model are fixed). We leave the details as an exercise.

Before we design the controller, let us verify that without control it is indeed the case that $\pi_{l}(H) \rightarrow \pm \hbar \omega_{0} / 2$ a.s. This already demonstrates the Lyapunov theorem.

Lemma 9.3. If $u_{l}^{\mu}=0$, then $\operatorname{Tr}\left[\rho_{l}^{\mu} \sigma_{z}\right] \rightarrow \pm 1$ with unit probability.

Proof. We will use the Lyapunov function $V\left(\rho_{l}^{\mu}\right)=1-\operatorname{Tr}\left[\rho_{l}^{\mu} \sigma_{z}\right]^{2}$, which is nonnegative and is zero precisely when $\operatorname{Tr}\left[\rho_{l}^{\mu} \sigma_{z}\right]= \pm 1$. Using the filtering equation with $u_{l}^{\mu}=0$ and (8.8), we find that

$$
\Delta \operatorname{Tr}\left[\rho_{l}^{\mu} \sigma_{z}\right]=\frac{\sin (2 \lambda)}{\lambda} \frac{V\left(\rho_{l-1}^{\mu}\right)}{\cos ^{2}(2 \lambda)+\sin ^{2}(2 \lambda) V\left(\rho_{l-1}^{\mu}\right)} \Delta \tilde{y}_{l}^{\mu},
$$

where $\tilde{y}_{l}^{\mu}$ is the innovations process. Using the quantum Itô rule,

$$
\Delta V\left(\rho_{l}^{\mu}\right)=-\frac{\sin ^{2}(2 \lambda)}{\lambda^{2}} \frac{V\left(\rho_{l-1}^{\mu}\right)^{2}}{\left(\cos ^{2}(2 \lambda)+\sin ^{2}(2 \lambda) V\left(\rho_{l-1}^{\mu}\right)\right)^{2}}\left(\Delta \tilde{y}_{l}^{\mu}\right)^{2}+(\cdots) \Delta \tilde{y}_{l}^{\mu} .
$$

But $\mathbf{E}^{\mu}\left(\Delta \tilde{y}_{l}^{\mu} \mid \mathscr{Y}_{l-1}^{\mu}\right)=0$ by the martingale property, and furthermore

$$
\mathbf{E}^{\mu}\left(\left(\Delta \tilde{y}_{l}^{\mu}\right)^{2} \mid \mathscr{Y}_{l-1}^{\mu}\right)=\left(1-\lambda^{2} \operatorname{Tr}\left[\overline{\mathcal{J}}\left(\rho_{l-1}^{\mu}\right)\right]^{2}\right) \Delta t=\left(\cos ^{2}(2 \lambda)+\sin ^{2}(2 \lambda) V\left(\rho_{l-1}^{\mu}\right)\right) \Delta t,
$$

where we have used $(\Delta \tilde{Y}(l))^{2}=(\cdots) \Delta \tilde{Y}(l)+(\Delta Y(l))^{2}-(\Delta C(l))^{2}$ as in the proof of Lemma 5.8. Hence we find

$$
\mathbf{E}^{\mu}\left(\Delta V\left(\rho_{l}^{\mu}\right) \mid \sigma\left\{\rho_{l-1}^{\mu}\right\}\right)=-\frac{\sin ^{2}(2 \lambda)}{\lambda^{2}} \frac{V\left(\rho_{l-1}^{\mu}\right)^{2}}{\cos ^{2}(2 \lambda)+\sin ^{2}(2 \lambda) V\left(\rho_{l-1}^{\mu}\right)} \Delta t \leq 0 .
$$

The lemma now follows from the Lyapunov theorem.

9.2. Construction of a Lyapunov Control. We now turn to the control design. We wish to find a (time-invariant) feedback control $u_{l}^{\mu}=g\left(\rho_{l-1}^{\mu}\right)$ so that $\mathbb{P}\left(\pi_{l}^{\mu}\left(\sigma_{z}\right)\right) \rightarrow+1$ as $l \rightarrow \infty$. The way we approach this problem is to use a trial Lyapunov function $V(\rho)$ without fixing the control $g$. By inspecting the expression for $\mathbf{E}^{\mu}\left(\Delta V\left(\rho_{l}^{\mu}\right) \mid \sigma\left\{\rho_{l-1}^{\mu}\right\}\right)$, we can subsequently try to choose $g$ so that this expression is nonpositive and is zero only at (or in a small neighborhood of) the point $\tilde{\rho} \in S^{1}$, where $\operatorname{Tr}\left[\tilde{\rho} \sigma_{z}\right]=+1$. The desired result follows by dominated convergence. 
Let us implement this procedure. Choose the trial Lyapunov function $V(\tilde{\rho})=$ $1-\operatorname{Tr}\left[\tilde{\rho} \sigma_{z}\right]$. Using the filtering equation with $u_{l}^{\mu}=g\left(\rho_{l-1}^{\mu}\right),(8.8)$, and the martingale property of the innovations, we find that

$$
\begin{aligned}
& \mathbf{E}^{\mu}\left(\Delta V\left(\rho_{l}^{\mu}\right) \mid \sigma\left\{\rho_{l-1}^{\mu}\right\}\right)=-\frac{\sin \left(2 \lambda w\left(f\left(\rho_{l-1}^{\mu}\right)\right)\right)}{\lambda w\left(f\left(\rho_{l-1}^{\mu}\right)\right)} f\left(\rho_{l-1}^{\mu}\right) \operatorname{Tr}\left[\rho_{l-1}^{\mu}\left(\sigma_{+}+\sigma_{-}\right)\right] \Delta t \\
& +\frac{2 f\left(\rho_{l-1}^{\mu}\right)^{2} \sin ^{2}\left(\lambda w\left(f\left(\rho_{l-1}^{\mu}\right)\right)\right)}{w\left(f\left(\rho_{l-1}^{\mu}\right)\right)^{2}} \operatorname{Tr}\left[\rho_{l-1}^{\mu} \sigma_{z}\right] \Delta t .
\end{aligned}
$$

As a first attempt, consider the following feedback function:

$$
f(\tilde{\rho})= \begin{cases}-1 & \text { if } \operatorname{Tr}\left[\tilde{\rho}\left(\sigma_{+}+\sigma_{-}\right)\right]<0 \\ +1 & \text { if } \operatorname{Tr}\left[\tilde{\rho}\left(\sigma_{+}+\sigma_{-}\right)\right] \geq 0\end{cases}
$$

Then

$\frac{\mathbf{E}^{\mu}\left(\Delta V\left(\rho_{l}^{\mu}\right) \mid \sigma\left\{\rho_{l-1}^{\mu}\right\}\right)}{\Delta t}=-\frac{\sin (2 \lambda w(1))}{\lambda w(1)}\left|\operatorname{Tr}\left[\rho_{l-1}^{\mu}\left(\sigma_{+}+\sigma_{-}\right)\right]\right|+\frac{2 \sin ^{2}(\lambda w(1))}{w(1)^{2}} \operatorname{Tr}\left[\rho_{l-1}^{\mu} \sigma_{z}\right]$.

Keeping in mind that $\rho_{l}^{\mu} \in S^{1}$ for all $l$, clearly there exists some $\delta>0$ such that this expression is strictly negative for $\operatorname{Tr}\left[\rho_{l-1}^{\mu} \sigma_{z}\right]<1-\delta$; in fact, $\delta$ is the solution of

$$
-\frac{\sin (2 \lambda w(1))}{\lambda w(1)} \sqrt{(2-\delta) \delta}+\frac{2 \sin ^{2}(\lambda w(1))}{w(1)^{2}}(1-\delta)=0 .
$$

To keep $\mathbf{E}^{\mu}\left(\Delta V\left(\rho_{l}^{\mu}\right) \mid \sigma\left\{\rho_{l-1}^{\mu}\right\}\right)$ nonpositive everywhere, we now modify the control function to turn off the feedback in the set $\operatorname{Tr}\left[\rho_{l-1}^{\mu} \sigma_{z}\right] \geq 1-\delta$ :

$$
g(\tilde{\rho})= \begin{cases}0 & \text { if } \operatorname{Tr}\left[\tilde{\rho} \sigma_{z}\right] \geq 1-\delta \\ -1 & \text { if } \operatorname{Tr}\left[\tilde{\rho}\left(\sigma_{+}+\sigma_{-}\right)\right]<0 \text { and } \operatorname{Tr}\left[\tilde{\rho} \sigma_{z}\right]<1-\delta \\ +1 & \text { if } \operatorname{Tr}\left[\tilde{\rho}\left(\sigma_{+}+\sigma_{-}\right)\right] \geq 0 \text { and } \operatorname{Tr}\left[\tilde{\rho} \sigma_{z}\right]<1-\delta\end{cases}
$$

This ensures that $\mathbf{E}^{\mu}\left(\Delta V\left(\rho_{l}^{\mu}\right) \mid \sigma\left\{\rho_{l-1}^{\mu}\right\}\right) \leq 0$, where the equality holds only when $\operatorname{Tr}\left[\rho_{l-1}^{\mu} \sigma_{z}\right] \geq 1-\delta$. The Lyapunov theorem then guarantees that $\operatorname{Tr}\left[\rho_{l}^{\mu} \sigma_{z}\right]$ converges to the set $[1-\delta, 1]$; i.e., $\liminf _{l \rightarrow \infty} \operatorname{Tr}\left[\rho_{l}^{\mu} \sigma_{z}\right] \geq 1-\delta$ with unit probability. For $\lambda \ll 1$, the threshold $\delta$ will be very small and hence the Lyapunov theorem guarantees convergence to a tiny neighborhood of the desired control goal. For example, with the choice $\lambda^{-2}=300$, which we have used in the simulations, $\delta \approx 5.6 \times 10^{-6}$.

Remark 9.4. We can do better than proving convergence to a small neighborhood of the target point. In addition to the convergence $k\left(x_{t}\right) \rightarrow 0$, the invariant set theorems [62] tell us that $x_{t}$ itself converges to the largest invariant set contained in $\{x: k(x)=0\}$ (the convergence is in probability, but this is easily strengthened to a.s. convergence). As the only invariant set inside the set $\operatorname{Tr}\left[\tilde{\rho} \sigma_{z}\right] \geq 1-\delta$ is the target point $\operatorname{Tr}\left[\tilde{\rho} \sigma_{z}\right]=1$, convergence is guaranteed. A full discussion of the required theorems is beyond the scope of this article, and we refer to [62] for further details.

To illustrate the effectiveness of (9.1), we have used the Monte Carlo method to simulate in Figure 9.1 several sample paths of the controlled filter. It is immediately evident that the control goal was attained for the five sample paths plotted, and the Lyapunov theorem guarantees that this is indeed the case for any sample path.

The discussion above is mainly intended as an illustration of an alternative to optimal control theory, and we have only used the Lyapunov theory in its simplest 

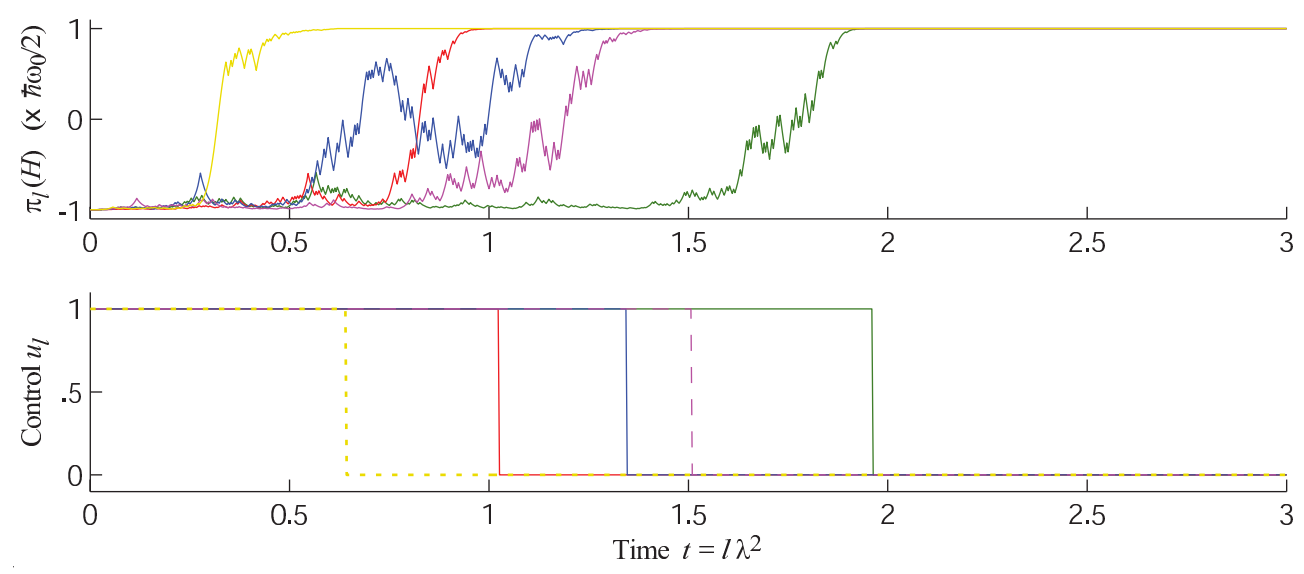

Fig. 9.I Five typical sample paths of the conditional expectation $\pi_{l}(H)$ of the atomic energy (top plot) for the controlled quantum flow on the circle with separated feedback law (9.1). Note that $\pi_{l}(H)=+\hbar \omega_{0} / 2$ is always attained. The bottom plot shows the feedback signal $u_{l}^{\mu}=$ $g\left(\rho_{l-1}^{\mu}\right)$. The initial state was $\rho(x)=\langle\Phi, X \Phi\rangle, \rho \in S^{1}$, the time scale is $\lambda^{-2}=300$, and $\delta=6 \times 10^{-6}$.

form. Lyapunov methods can be extended to a large class of systems; see, e.g., [69], whose treatment using optimal control would be out of the question due to the high dimensionality of the state space. Some control goals other than strict convergence can be treated using similar methods; for example, we could try to find a control law so that a quantity such as $\mathbf{E}\left(\left|\operatorname{Tr}\left[\rho_{l}^{\mu} \sigma_{z}\right]-\alpha\right|\right)$ for some $\alpha \in(-1,1)$ becomes small (but usually nonzero) as $l \rightarrow \infty$; see, e.g., [86]. Beside the restricted set of control goals that can be treated by Lyapunov methods, a drawback of such methods is that there is no general recipe which one can follow for the design of controls; the choice of a proper control and Lyapunov function has to be investigated on a case-by-case basis. Ultimately the control goal of interest and the available resources determine which method of control design - be it optimal control, Lyapunov methods, or some other approach - is most suitable for the problem at hand.

10. References for Further Reading. We have come to the end of our exposition on the filtering and control of discrete quantum models, though in many ways we have only scratched the surface of the theory of quantum probability, filtering, and stochastic control. The goal of this final section is to provide some entry points into the literature for further reading.

Quantum Probability. We have used quantum probability theory in its simplest finite-dimensional form, where only finite state random variables are available. In practice such a description is very restrictive, and one needs a theory that admits continuous random variables. The theory of operator algebras (e.g., Kadison and Ringrose [53, 54] or Bratteli and Robinson [21]), particularly von Neumann algebras, provides the proper generalization of the concept of a quantum probability space to the infinite-dimensional context. For an accessible introduction to quantum probability we refer to the excellent lecture notes by Maassen [65]. See also [16, 20] and the references therein. The required background on functional analysis can be found, e.g., in the textbook by Reed and Simon [74]. 
Readers with a background in physics might wonder why we did not use the projection postulate in this paper. We do not need this postulate to develop the theory, and in fact the projection postulate can be obtained as a special case within the quantum probability framework used in this paper. See [20] for an example.

Quantum Noise and Stochastic Calculus. The three discrete noises - we have suggestively denoted them as $A(l), A^{*}(l)$, and $\Lambda(l)$-are replaced in the continuous time theory by the standard noises $A_{t}$ (the annihilation process), $A_{t}^{*}$ (the creation process), and $\Lambda_{t}$ (the gauge process). For each time $t, A_{t}, A_{t}^{*}, \Lambda_{t}$ are defined as operators acting on a Hilbert space that is known as the Fock space, and the latter plays a central role in the theory of quantum noise. The processes $\left(A_{t}+A_{t}^{*}\right)_{t \geq 0}$ or $\left(\Lambda_{t}\right)_{t \geq 0}$ each generate a commutative algebra; the spectral theorem allows us to interpret the former as a diffusion process (a Wiener process in the vacuum state), and the latter as a counting process (a Poisson process in a so-called coherent state, and a.s. zero in the vacuum). A brief introduction is given in, e.g., [20].

Based on these processes, one can now proceed to define quantum stochastic integrals and obtain the quantum Itô rule: $d A_{t} d A_{t}^{*}=d t, d \Lambda_{t} d A_{t}^{*}=d A_{t}^{*}, d A_{t} d \Lambda_{t}=d A_{t}$, and all other combinations are zero. Next one defines quantum stochastic differential equations, which provide "quantum noisy" versions of the famous Schrödinger equation and form good models of actual physical systems, particularly those used in quantum optics (see [36] for a physicist's perspective). An accessible starting point in the literature on quantum stochastic calculus are Hudson's lecture notes [48]; see also the original article by Hudson and Parthasarathy [49] and Parthasarathy's book [71]. Several other approaches to the theory have been developed since; some of these appear in the book by Meyer [68] and the lecture notes of Biane [16].

Physical Models. The physical theory that describes the interaction of light (the electromagnetic field) with matter (atoms, molecules, etc.) is called quantum electrodynamics; see the book by Cohen-Tannoudji, Dupont-Roc, and Grynberg [25]. Very often, and in particular for the purposes of filtering and control, quantum electrodynamics does not directly provide a usable model; these models are non-Markov. In many systems, however, the Markov approximation is quite good, and applying this approximation to models from quantum electrodynamics gives rise precisely to quantum stochastic differential equations. The Markov approximation can be pursued at different levels of rigor, ranging from the ad-hoc "whitening" of the noise which is common in the physics literature [36] to rigorous Wong-Zakai-type limits [42, 1]. As in classical probability theory it is also common to begin with a Markov model in the form of a quantum stochastic differential equation, which is justified either on phenomenological grounds or through a separate modeling effort.

The modeling of the detectors, e.g., photodetection or homodyne detection, is another important topic. The operating principles of optical detectors are described in many textbooks on quantum optics; see, e.g., [81, 66]. In the quantum stochastic context we refer to, e.g., [36], or to the more mathematical perspective of Barchielli [7].

Continuous Limits of Discrete Models. The discrete models which we have used throughout the paper are conceptually very similar to the more realistic continuous models. There is more than a conceptual resemblance, however: these models in fact converge to corresponding continuous models as we let the time step $\lambda^{2} \rightarrow 0$. Though there is no particular physical intuition behind this convergence except the one we have already given - that small time slices of the field approximately contain at most one photon - the convergence demonstrates that even results obtained using these highly simplified models have some relevance to the physical world. 
A simple example of this convergence is easily verified. Recall that the classical stochastic process $x(l)=\iota\left(A(l)+A^{*}(l)\right)$, with the measure induced by the vacuum state, defines a symmetric random walk with step size $\lambda$. First, let us express this process in terms of time $t=l \lambda^{2}$ rather than the time step number $l$, i.e., $x_{t}=$ $x\left(\left\lfloor t / \lambda^{2}\right\rfloor\right)$. Taking the limit (in law) as $\lambda \rightarrow 0$ gives, by the usual functional central limit argument (e.g., [39, p. 452]), a Wiener process. But $\iota\left(A_{t}+A_{t}^{*}\right)$ is a Wiener process, so we see that $A(l)+A^{*}(l)$ converges to $A_{t}+A_{t}^{*}$ at least in a weak sense.

Similarly, most of the expressions given in this article converge as $\lambda \rightarrow 0$ to their continuous time counterparts. In particular the difference equation (4.1), the filtering equations, etc., converge to (quantum) stochastic differential equations. Even the discrete Itô table "converges" to the quantum Itô rules $d A_{t} d A_{t}^{*}=d t$, etc., if we formally set $\Delta \Lambda \rightarrow d \Lambda, \Delta A \rightarrow d A, \Delta A^{*} \rightarrow d A^{*}, \Delta t \rightarrow d t$, and terms such as $\lambda^{2} \Delta A \rightarrow 0$ (this was noticed in [5, 72]). Evidently the discrete theory mirrors its continuous counterpart quite faithfully, which is the motivation for this article.

Convergence of the discrete models was investigated by Lindsay and Parthasarathy [63], Attal and Pautrat [6], and Gough [41]. These articles demonstrate convergence (of various types) of the solution of the difference equation (4.1) to the solution of a corresponding quantum stochastic differential equation. The convergence of discrete filters to their continuous counterparts was investigated in Gough and Sobolev [43].

Conditional Expectations and Quantum Filtering. The concept of conditional expectations in von Neumann algebras is an old one; see, e.g., [79]. However, a much more pragmatic notion of conditional expectations on which the definition we have given is based first appeared in the pioneering work of Belavkin; see, e.g., [12]. The necessity of the nondemolition property, which is the key to the development of both filtering and feedback control, appeared in [8]. This opened the door for the development of nonlinear filtering, which is described in detail in the difficult and technical paper [12] using martingale methods. The reference probability approach to quantum filtering, using the Bayes formula, appears in [17] (but see also [11, 40]). An introduction to quantum filtering is given in [20] using both methods.

Feedback Control. An early investigation on optimal feedback control in discrete time can be found in Belavkin's 1983 paper [9]. The Bellman equation for continuous optimal control appears in [10]. A recent exposition on quantum optimal control, and in particular quantum LQG control, is given in [33]; see also [18], and see [30, 31] for a physicist's perspective. The separation theorem for the continuous time case appears in [17]. A different type of optimal control problem, the risk-sensitive control problem with exponential cost, is investigated in [50]. Finally, Lyapunov function methods for control design are investigated in [80,69].

Many applications of quantum feedback control have been considered in the physics literature; we refer to the introduction for references. Note that the "quantum trajectory equations" or "stochastic master equations" used in the physics literature are precisely filtering equations, written in terms of the innovations process as the driving noise rather than being driven by the observations. The notion of filtering has only recently gained acceptance in the physics literature, though the relevant equations had already been developed by physicists. The reader should beware of the discrepancy in terminology and interpretation between the physical and mathematical literature. However, as the Markov property of the filter allows us to pretend that the filter itself is the system to be controlled, most of the control schemes found in the physics literature make sense in both contexts. 
Acknowledgments. The authors would like to thank John Stockton for providing a figure. L.B. thanks Hans Maassen for introducing him to discrete systems.

\section{REFERENCES}

[1] L. Accardi, J. Gough, And Y. Lu, On the stochastic limit for quantum theory, Rep. Math. Phys., 36 (1995), pp. 155-187.

[2] A. André, A. S. Sørensen, And M. D. Lukin, Stability of atomic clocks based on entangled atoms, Phys. Rev. Lett., 92 (2004), article 230801.

[3] M. A. Armen, J. K. Au, J. K. Stockton, A. C. Doherty, and H. Mabuchi, Adaptive homodyne measurement of optical phase, Phys. Rev. Lett., 89 (2002), article 133602.

[4] K. Astrom, Introduction to Stochastic Control Theory, Academic Press, New York, 1970.

[5] S. Attal, Approximating the Fock space with the toy Fock space, in Séminaire de Probabilités, XXXVI, Lecture Notes in Math. 1801, Springer, Berlin, 2003, pp. 477-491.

[6] S. Attal and Y. Pautrat, From repeated to continuous quantum interactions, Ann. Henri Poincaré, 7 (2006), pp. 59-104.

[7] A. Barchielli, Continual measurements in quantum mechanics and quantum stochastic calculus, in Open Quantum Systems III: Recent Developments, S. Attal, A. Joye, and C.-A. Pillet, eds., Springer, Berlin, 2006, pp. 207-292.

[8] V. P. Belavkin, Quantum filtering of Markov signals with white quantum noise, Radio Engrg. Electron. Phys., 25 (1980), pp. 1445-1453.

[9] V. P. Belavkin, Theory of the control of observable quantum systems, Automat. Remote Control, 44 (1983), pp. 178-188.

[10] V. P. BelaVkin, Nondemolition stochastic calculus in Fock space and nonlinear filtering and control in quantum systems, in Proceedings XXIV Karpacz winter school, R. Guelerak and W. Karwowski, eds., Stochastic Methods in Mathematics and Physics, World Scientific, Singapore, 1988, pp. 310-324.

[11] V. P. Belavkin, Quantum continual measurements and a posteriori collapse on CCR, Commun. Math. Phys., 146 (1992), pp. 611-635.

[12] V. P. Belavkin, Quantum stochastic calculus and quantum nonlinear filtering, J. Multivar. Anal., 42 (1992), pp. 171-201.

[13] A. Bensoussan, Stochastic Control of Partially Observable Systems, Cambridge University Press, Cambridge, UK, 1992.

[14] D. Bertsekas, Dynamic Programming and Optimal Control, Vol. 1, Athena Scientific, Boston, 1995.

[15] D. Bertsekas and S. Shreve, Stochastic Optimal Control: The Discrete-Time Case, Academic Press, New York, 1978.

[16] P. Biane, Calcul stochastique noncommutatif, in Lectures on Probability Theory (Saint-Flour, 1993), P. Bernard, ed., Lecture Notes in Math. 1608, Springer, Berlin, 1995, pp. 1-96.

[17] L. Bouten And R. van Handel, On the separation principle of quantum control, in Quantum Stochastics and Information: Statistics, Filtering and Control, V. P. Belavkin and M. Guta, eds., World Scientific, London, 2008, pp. 206-238.

[18] L. M. Bouten, S. C. Edwards, and V. P. Belavkin, Bellman equations for optimal feedback control of qubit states, J. Phys. B, At. Mol. Opt. Phys., 38 (2005), pp. 151-160.

[19] L. M. Bouten, M. I. Guţă, And H. MAassen, Stochastic Schrödinger equations, J. Phys. A, 37 (2004), pp. 3189-3209.

[20] L. Bouten, R. Van Handel, and M. R. James, An introduction to quantum filtering, SIAM J. Control Optim., 46 (2007), pp. 2199-2241.

[21] O. Bratteli and D. Robinson, Operator Algebras and Quantum Statistical Mechanics 1, 2nd ed., Springer, Berlin, Heidelberg, 1987.

[22] T. A. Brun, A simple model of quantum trajectories, Amer. J. Phys., 70 (2002), pp. 719-737.

[23] P. Bushev, D. Rotter, A. Wilson, F. Dubin, C. Becher, J. Eschner, R. Blatt, V. Steixner, P. RABl, And P. Zoller, Feedback cooling of a single trapped ion, Phys. Rev. Lett., 96 (2006), article 043003.

[24] H. J. Carmichael, An Open Systems Approach to Quantum Optics, Springer, Berlin, Heidelberg, New York, 1993.

[25] C. Cohen-Tannoudji, J. Dupont-Roc, and G. Grynberg, Photons and Atoms: Introduction to Quantum Electrodynamics, Wiley, New York, 1989.

[26] J. C. Cox, S. A. Ross, And M. Rubinstein, Option pricing: A simplified approach, J. Financial Econom., 7 (1979), pp. 229-263.

[27] E. B. Davies, Quantum Theory of Open Systems, Academic Press, London, New York, San Francisco, 1976.

Copyright (c) by SIAM. Unauthorized reproduction of this article is prohibited. 
[28] E. B. DAviES, Quantum communication systems, IEEE Trans. Inform. Theory, IT-23 (1977), pp. $530-534$.

[29] M. H. A. Davis and S. I. Marcus, An introduction to nonlinear filtering, in Stochastic Systems: The Mathematics of Filtering and Identification and Applications, M. Hazewinkel and J. C. Willems, eds., D. Reidel, Boston, 1981, pp. 53-75.

[30] A. C. DoherTy And K. JacoBs, Feedback-control of quantum systems using continuous stateestimation, Phys. Rev. A, 60 (1999), pp. 2700-2711.

[31] A. C. Doherty, S. Habib, K. Jacobs, H. Mabuchi, and S. M. Tan, Quantum feedback and classical control theory, Phys. Rev. A, 62 (2000), article 012105.

[32] T. Duncan, Evaluation of likelihood functions, Inform. Control, 13 (1968), pp. 62-74.

[33] S. C. Edwards and V. P. Belavkin, Optimal Quantum Feedback Control via Quantum Dynamic Programming, University of Nottingham, 2005; available online from http://arxiv. org/abs/quant-ph/0506018v2.

[34] R. J. Elliott, L. Aggoun, and J. B. Moore, Hidden Markov Models: Estimation and Control, Springer, New York, 1995.

[35] W. Fleming and R. Rishel, Deterministic and Stochastic Optimal Control, Springer, New York, 1975.

[36] C. Gardiner and P. Zoller, Quantum Noise, 3rd ed., Springer, Berlin, 2004.

[37] J. M. Geremia, Distinguishing between optical coherent states with imperfect detection, Phys. Rev. A, 70 (2004), article 062303.

[38] J. M. Geremia, J. K. Stockton, and H. Mabuchi, Suppression of spin projection noise in broadband atomic magnetometry, Phys. Rev. Lett., 94 (2005), article 203002.

[39] I. I. Gikhman and A. V. Skorokhod, Introduction to the Theory of Random Processes, Dover, New York, 1996.

[40] P. Goetsch And R. Graham, Linear stochastic wave equations for continuously measured quantum systems, Phys. Rev. A, 50 (1994), pp. 5242-5255.

[41] J. Gough, Holevo-ordering and the continuous-time limit for open Floquet dynamics, Lett. Math. Phys., 67 (2004), pp. 207-221.

[42] J. Gough, Quantum flows as Markovian limit of emission, absorption and scattering interactions, Commun. Math. Phys., 254 (2005), pp. 489-512.

[43] J. Gough And A. Sobolev, Stochastic Schrödinger equations as limit of discrete filtering, Open Syst. Inf. Dyn., 11 (2004), pp. 235-255.

[44] P. Halmos, Finite-Dimensional Vector Spaces, Springer, New York, 1974.

[45] A. Holevo, Quantum stochastic calculus, J. Soviet Math., 56 (1991), pp. 2609-2624. Translation of Itogi Nauki i Tekhniki, Ser. Sovr. Prob. Mat., 36 (1990), pp. 3-28.

[46] A. Hopkins, K. Jacobs, S. Habib, And K. Schwab, Feedback cooling of a nanomechanical resonator, Phys. Rev. B, 68 (2003), article 235328.

[47] R. A. Horn and C. R. Johnson, Matrix Analysis, Cambridge University Press, Cambridge, $\mathrm{UK}, 1985$

[48] R. L. Hudson, An introduction to quantum stochastic calculus and some of its applications, in Quantum Probability Communications, Vol. XI, S. Attal and J. Lindsay, eds., World Scientific, Singapore, 2003, pp. 221-271.

[49] R. L. Hudson and K. R. Parthasarathy, Quantum Itô's formula and stochastic evolutions, Commun. Math. Phys., 93 (1984), pp. 301-323.

[50] M. R. James, A quantum Langevin formulation of risk-sensitive optimal control, J. Opt. B Quantum Semiclass. Opt., 7 (2005), pp. S198-S207.

[51] M. James and I. Petersen, Robustness properties of a class of optimal risk-sensitive controllers for quantum systems, in Proceedings of the 16th IFAC World Congress, Prague, 2005.

[52] M. James, H. Nurdin, and I. Petersen, $H^{\infty}$ control of linear quantum systems, IEEE Trans. Automat. Control, 53 (2008), pp. 1787-1803.

[53] R. V. Kadison and J. R. Ringrose, Fundamentals of the Theory of Operator Algebras, Vol. I, Academic Press, San Diego, 1983.

[54] R. V. Kadison and J. R. Ringrose, Fundamentals of the Theory of Operator Algebras, Vol. II, Academic Press, San Diego, 1986.

[55] G. Kallianpur, Stochastic Filtering Theory, Springer, Berlin, 1980.

[56] G. Kallianpur and C. Striebel, Estimation of stochastic systems: Arbitrary system process with additive white noise observation errors, Ann. Math. Statist., 39 (1968), pp. 785-801.

[57] G. Kallianpur And C. Striebel, Stochastic differential equations occurring in the estimation of continuous parameter stochastic processes, Teor. Verojatnost. i Primenen, 14 (1969), pp. 597-622.

[58] N. Khaneja, T. Reiss, B. Luy, and S. J. Glaser, Optimal control of spin dynamics in the presence of relaxation, J. Magnet. Res., 162 (2003), pp. 311-319.

Copyright $@$ by SIAM. Unauthorized reproduction of this article is prohibited. 
[59] V. KRISHnan, Nonlinear Filtering and Smoothing: An Introduction to Martingales, Stochastic Integrals and Estimation, Dover, New York, 2005.

[60] P. Kumar and P. Varaiya, Stochastic Systems: Estimation, Identification and Adaptive Control, Prentice-Hall, Englewood Cliffs, NJ, 1986.

[61] B. Kümmerer, Markov dilations on $W^{*}$-algebras, J. Funct. Anal., 63 (1985), pp. 139-177.

[62] H. Kushner, Introduction to Stochastic Control, Holt, Rinehart, and Winston, New York, 1971.

[63] J. M. Lindsay and K. R. Parthasarathy, The passage from random walk to diffusion in quantum probability. II, Sankhyā Ser. A, 50 (1988), pp. 151-170.

[64] R. S. Liptser And A. N. Shiryaev, Statistics of Random Processes I: General Theory, Springer, Berlin, 2001.

[65] H. MAASSEN, Quantum probability applied to the damped harmonic oscillator, in Quantum Probability Communications, Vol. XII, S. Attal and J. Lindsay, eds., World Scientific, Singapore, 2003, pp. 23-58.

[66] L. Mandel And E. Wolf, Optical Coherence and Quantum Optics, Cambridge University Press, Cambridge, UK, 1995.

[67] E. Merzbacher, Quantum Mechanics, 3rd ed., Wiley, New York, 1998.

[68] P.-A. MeYer, Quantum Probability for Probabilists, Springer, Berlin, 1993.

[69] M. Mirrahimi and R. VAn Handel, Stabilizing feedback controls for quantum systems, SIAM J. Control Optim., 46 (2007), pp. 445-467.

[70] R. Mortensen, Optimal Control of Continuous-Time Stochastic Systems, Ph.D. thesis, University of California, Berkeley, 1966.

[71] K. R. Parthasarathy, An Introduction to Quantum Stochastic Calculus, Birkhäuser, Basel, 1992.

[72] Y. PaUtrat, From Pauli matrices to quantum Itô formula, Math. Phys. Anal. Geom., 8 (2005), pp. 121-155.

[73] P. E. Protter, Stochastic Integration and Differential Equations, 2nd ed., Springer, Berlin, 2004.

[74] M. Reed And B. Simon, Methods of Modern Mathematical Physics. I. Functional Analysis, Elsevier, New York, 1980.

[75] S. E. Shreve, Stochastic Calculus for Finance I. The Binomial Asset Pricing Model, Springer, New York, 2004.

[76] D. A. Steck, K. Jacobs, H. Mabuchi, T. Bhattacharya, and S. Habib, Quantum feedback control of atomic motion in an optical cavity, Phys. Rev. Lett., 92 (2004), article 223004.

[77] J. K. Stockton, Continuous Quantum Measurement of Cold Alkali-Atom Spins, Ph.D. thesis, California Institute of Technology, Pasadena, CA, 2006.

[78] J. K. Stockton, J. M. Geremia, A. C. Doherty, and H. Mabuchi, Robust quantum parameter estimation: Coherent magnetometry with feedback, Phys. Rev. A, 69 (2004), article 032109.

[79] M. TAKesaki, Conditional expectations in von Neumann algebras, J. Funct. Anal., 9 (1971), pp. 306-321.

[80] R. van Handel, J. K. Stockton, and H. Mabuchi, Feedback control of quantum state reduction, IEEE Trans. Automat. Control, 50 (2005), pp. 768-780.

[81] D. Walls and G. Milburn, Quantum Optics, Springer, Berlin, Heidelberg, 1994.

[82] P. Whittle, Risk-sensitive linear/quadratic/Gaussian control, Adv. in Appl. Probab., 13 (1981), pp. 764-777.

[83] D. Williams, Probability with Martingales, Cambridge University Press, Cambridge, UK, 1991.

[84] H. M. Wiseman, Adaptive phase measurements of optical modes: Going beyond the marginal $q$ distribution, Phys. Rev. Lett., 75 (1995), pp. 4587-4590.

[85] H. M. Wiseman And G. J. Milburn, All-optical versus electro-optical quantum-limited feedback, Phys. Rev. A, 49 (1994), pp. 4110-4125.

[86] N. Yamamoto, K. Tsumura, and S. Hara, Feedback control of quantum entanglement in a two-spin system, in Proceedings of the 44th IEEE CDC, Seville, 2005, pp. 3182-3187.

[87] M. YANAGiSAwA AND H. KimURA, Transfer function approach to quantum control-Part II: Control concepts and applications, IEEE Trans. Automat. Control, 48 (2003), pp. 21212132.

[88] M. ZAKAI, On the optimal filtering of diffusion processes, Z. Wahrscheinlichkeitstheorie und Verw. Gebiete, 11 (1969), pp. 230-243.

Copyright (c) by SIAM. Unauthorized reproduction of this article is prohibited. 